\title{
Standard and non-standard CAGD tools for isogeometric analysis: a tutorial
}

Carla Manni and Hendrik Speleers

Report TW642, January 2014

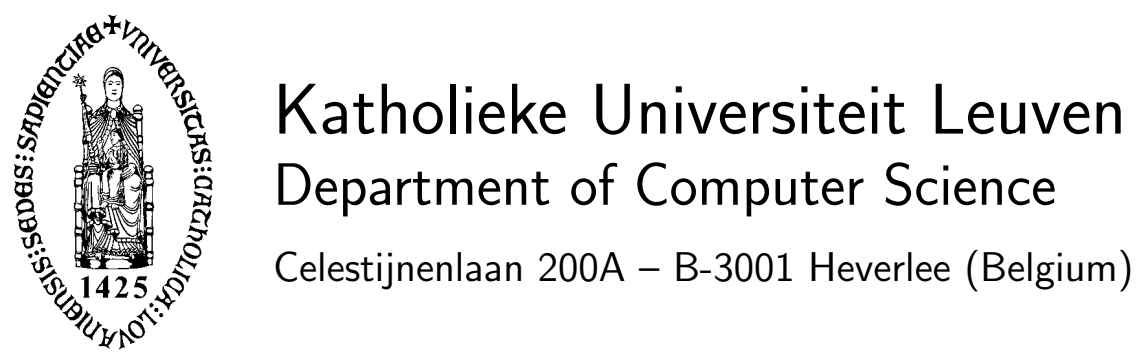




\title{
Standard and non-standard CAGD tools for isogeometric analysis: a tutorial
}

\author{
Carla Manni and Hendrik Speleers
}

Report TW642, January 2014

Department of Computer Science, KU Leuven

\begin{abstract}
We present a short summary of CAGD tools of main interest in isogeometric analysis, namely Bernstein polynomials and B-splines. Besides their well-known algebraic and geometric properties, we provide a deeper insight why these representations are so popular and efficient by proving that they are optimal bases for the corresponding function spaces. Moreover, we review some generalizations of the B-spline structure in function spaces which extend classical polynomials. Extensions to the bivariate case beyond the straightforward tensor-product case are also presented.
\end{abstract}

Keywords : Isogeometric analysis, Bernstein polynomials, B-splines, optimal bases, generalized splines, splines on triangulations 


\title{
Standard and Non-Standard CAGD Tools for Isogeometric Analysis: a Tutorial
}

Carla Manni and Hendrik Speleers

\begin{abstract}
We present a short summary of CAGD tools of main interest in $\operatorname{IgA}$, namely Bernstein polynomials and B-splines. Besides their well-known algebraic and geometric properties, we provide a deeper insight why these representations are so popular and efficient by proving that they are optimal bases for the corresponding function spaces. Moreover, we review some generalizations of the B-spline structure in function spaces which extend classical polynomials. Extensions to the bivariate case beyond the straightforward tensor-product case are also presented.
\end{abstract}

\section{Introduction}

Computer Aided Geometric Design (CAGD) is a well-established area of mathematics devoted to the representation and manipulation of curves and surfaces. Recently, this area has attracted a considerable interest from researchers working in numerical simulation due to the success of the emerging field of Isogeometric Analysis ( $\operatorname{IgA}$ ), see [19].

The aim of this chapter is to provide an extended summary of the most popular tools in CAGD, namely Bernstein polynomials and B-splines, and some possible generalizations for them.

There exists a large amount of literature on the above topics, including several well-established books [8, 34, 54, 59] and classical review papers [4]. However, our goal is twofold. First, we want to present the material from the IgA perspective point of view. Second, we aim to provide a deeper insight into the well-known al-

\section{Carla Manni}

Dipartimento di Matematica, Università di Roma "Tor Vergata" - Italy

e-mail: manni@mat.uniroma2.it

Hendrik Speleers

Departement Computerwetenschappen, Katholieke Universiteit Leuven - Belgium

e-mail: hendrik.speleers@cs.kuleuven.be 
gebraic and geometric properties of Bernstein polynomials and B-splines, in order to explain why these representations are so popular and efficient. More precisely, we will show that both Bernstein polynomials and B-splines are, in a suitable sense, the best bases to represent polynomials or piecewise polynomials, respectively. This optimality result deeply relies on the so-called total positivity property of the above functions. Despite its elegance, total positivity and its geometric consequences are not so common topics in CAGD tutorials.

Moreover, the total positivity point of view allows us to point out that the above optimality properties are not confined to the (piecewise) polynomial case, but rely on more general structural properties of the space. This paves the way for a natural generalization of the B-spline representation to a large class of spaces. This enriched environment can be exploited to build optimal representations for spaces of interest in IgA and related applications.

The most easy way to extend univariate results to the multivariate setting, is to consider a so-called tensor-product approach. Unfortunately, despite their simple and elegant formulation, tensor-product structures do not allow local refinements which are essential in numerical simulation. Therefore, we also aim to introduce appropriate tools supporting local refinement. To this end we provide some results for surface representations beyond the classical tensor-product approach. More precisely, since triangulations naturally support local mesh refinements, we discuss the Bernstein representation for polynomials on triangles and B-spline-like structures for suitable spline spaces on triangulations.

The remaining part of this chapter is organized as follows.

In Sect. 2 we describe the Bernstein polynomials and Bézier curves with their classical properties. Moreover, we introduce the concepts of total positivity and optimal bases. We prove that Bernstein polynomials provide the optimal representation for algebraic polynomials.

Section 3 is devoted to B-splines, B-spline curves and (tensor-product) B-spline surfaces, and their rational extension (NURBS). First, we review their main algebraic and geometric properties. Then, following the same approach as for the polynomial case, we pay attention to their total positivity and optimality properties and we prove that B-splines provide the optimal representation for piecewise polynomial spaces with certain smoothness.

In Sect. 4 we focus on a natural generalization of the Bernstein/B-spline basis to a larger class of function spaces, namely the extended Chebyshev spaces. We define and analyze the so-called generalized B-splines which maintain the same properties as classical algebraic B-splines.

Section 5 presents Bernstein polynomials on triangles and B-splines on suitable triangulations. We pay special attention to the so-called Powell-Sabin B-splines.

Finally, we end in Sect. 6 with some final remarks concerning a possible use in IgA of the non-standard CAGD tools we have presented. 


\section{Bézier-Bernstein Representations in 1D}

In this section we focus on one of the most famous tools in CAGD: polynomial curves represented with Bernstein polynomials, well-known as Bézier curves. In addition to the definition and main properties of Bézier curves, we also provide a deeper insight into fundamental properties as total positivity and optimality to explain why the Bernstein polynomials are such a popular and efficient tool in CAGD. Moreover, we show that the Bernstein polynomials form the best basis to represent polynomials and polynomial curves.

\subsection{Bernstein Polynomials}

In this subsection we introduce Bernstein polynomials ${ }^{1}$ and we derive their most popular algebraic and geometric properties.

\subsubsection{Definition and Main Properties}

Definition 1. Bernstein polynomials of degree $p$ are given by

$$
B_{i}^{(p)}(t):=\left(\begin{array}{c}
p \\
i
\end{array}\right) t^{i}(1-t)^{p-i}, \quad i=0, \ldots, p .
$$

Figure 1 depicts the four cubic Bernstein polynomials $(p=3)$ on $[0,1]$. The Bernstein polynomials of degree $p$ form a basis for the space $\mathbb{P}_{p}$ of algebraic polynomials of degree $p$. Assuming ${ }^{2} t \in[0,1]$, they possess the following interesting properties.

- Positivity. All Bernstein polynomials are positive on $[0,1]$, i.e.,

$$
B_{i}^{(p)}(t) \geq 0, \quad t \in[0,1] .
$$

${ }^{1}$ Bernstein polynomials were introduced in [2] to provide a constructive proof of the Weierstrass theorem, i.e. to explicitly construct a sequence of algebraic polynomials, namely

$$
B_{p}(f, t):=\sum_{i=0}^{p} f\left(\frac{i}{p}\right)\left(\begin{array}{c}
p \\
i
\end{array}\right) t^{i}(1-t)^{p-i}
$$

which uniformly converges on $[0,1]$ to any $f \in C([0,1])$. From the approximation point of view, the above operator did not receive a lot of attention due to its slow convergence. Indeed, it can be proved that (see [2])

$$
\lim _{p \rightarrow \infty} p\left[B_{p}\left(f, t_{0}\right)-f\left(t_{0}\right)\right]=\frac{1}{2} t_{0}\left(1-t_{0}\right) f^{\prime \prime}\left(t_{0}\right), \quad \text { if } f^{\prime \prime}\left(t_{0}\right) \neq 0 .
$$

We refer to the nice paper [26] for a historical summary of the properties of Bernstein polynomials. ${ }^{2}$ A set of polynomials with the same properties on a general interval $[a, b]$ can be immediately obtained by the usual change of variable $t=\frac{x-a}{b-a}$. 


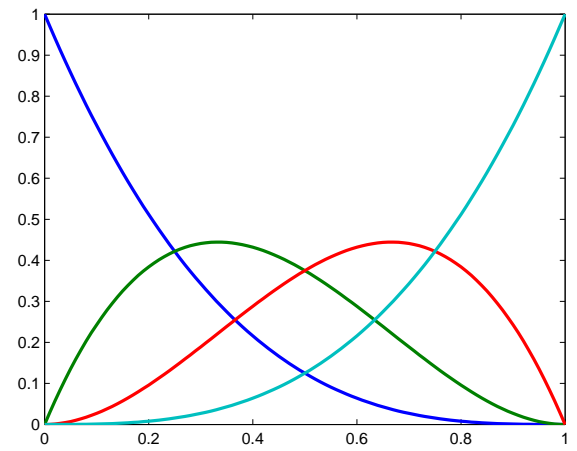

Fig. 1 Cubic Bernstein polynomials.

- Partition of unity. The Bernstein polynomials of the same degree sum up to one, i.e.,

$$
\sum_{i=0}^{p} B_{i}^{(p)}(t)=(t+1-t)^{p}=1, \quad \forall t \in \mathbb{R} .
$$

- Recurrence relation. Any Bernstein polynomial of degree $p$ can be written in terms of two consecutive Bernstein polynomials of degree $p-1$ as

$$
B_{i}^{(p)}(t)=(1-t) B_{i}^{(p-1)}(t)+t B_{i-1}^{(p-1)}(t)
$$

with $B_{i}^{(k)}=0$ if $i<0$ or $i>k$.

- Degree elevation (degree raising). Since $\mathbb{P}_{p} \subset \mathbb{P}_{p+1}$, a Bernstein polynomial of degree $p$ can be exactly represented as a positive combination of two consecutive Bernstein polynomials of degree $p+1$. More precisely,

$$
B_{i}^{(p)}(t)=(t+1-t) B_{i}^{(p)}(t)=\frac{i+1}{p+1} B_{i+1}^{(p+1)}(t)+\frac{p+1-i}{p+1} B_{i}^{(p+1)}(t) .
$$

- Derivatives.

- first derivative: the derivative of a Bernstein polynomial can be simply expressed in terms of two consecutive Bernstein polynomials of lower degree as

$$
\frac{\mathrm{d} B_{i}^{(p)}(t)}{\mathrm{d} t}=p\left[B_{i-1}^{(p-1)}(t)-B_{i}^{(p-1)}(t)\right] ;
$$

- end derivatives: the derivatives of Bernstein polynomials at the end points have the following behavior:

$$
\frac{\mathrm{d}^{r} B_{i}^{(p)}(0)}{\mathrm{d} t^{r}}=0, \quad r=0, \ldots, i-1,
$$




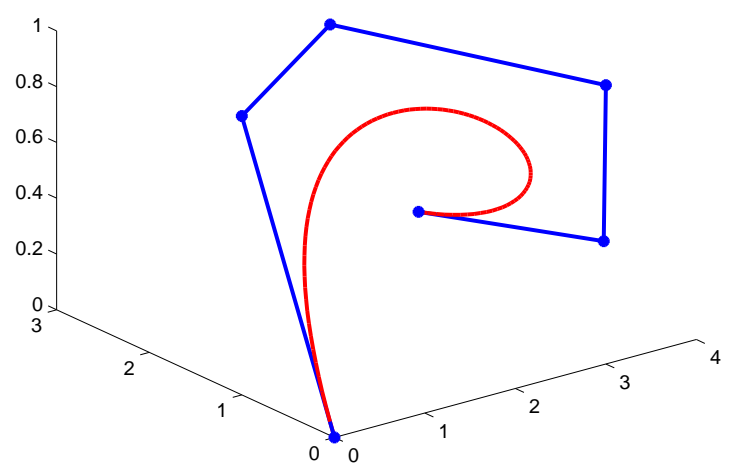

Fig. 2 A quintic Bézier curve and its control polygon.

and

$$
\frac{\mathrm{d}^{r} B_{i}^{(p)}(1)}{\mathrm{d} t^{r}}=0, \quad r=0, \ldots, p-i-1 ;
$$

- unimodal behavior: the interior Bernstein polynomials have a single extremum on the open interval $(0,1)$, and more precisely, for $i=1, \ldots, p-1$

$$
\frac{\mathrm{d} B_{i}^{(p)}(t)}{\mathrm{d} t}=0, t \in(0,1) \quad \text { if and only if } \quad t=\frac{i}{p}
$$

- Integration. All Bernstein polynomials of the same degree have the same integral on $[0,1]$, namely

$$
\int_{0}^{1} B_{i}^{(p)}(s) \mathrm{d} s=\frac{1}{p+1} .
$$

This can be shown as follows. Integrating the derivative formula (6) gives

$$
0=B_{i}^{(p)}(1)-B_{i}^{(p)}(0)=\int_{0}^{1} \frac{\mathrm{d} B_{i}^{(p)}(s)}{\mathrm{d} s} \mathrm{~d} s=p\left[\int_{0}^{1} B_{i-1}^{(p-1)}(s) \mathrm{d} s-\int_{0}^{1} B_{i}^{(p-1)}(s) \mathrm{d} s\right],
$$

for any $i=1, \ldots, p-1$. Thus,

$$
\int_{0}^{1} B_{i-1}^{(p-1)}(s) \mathrm{d} s=\int_{0}^{1} B_{i}^{(p-1)}(s) \mathrm{d} s .
$$

The Bernstein polynomials of degree $p$ sum up to one and there are $p+1$ of them, so we may deduce (10). 
Example 1. By integrating the relation (6), we obtain the integral recurrence relation

$$
\begin{aligned}
& B_{0}^{(p)}(t)=1-d_{0}^{(p-1)} \int_{0}^{t} B_{0}^{(p-1)}(s) \mathrm{d} s \\
& B_{i}^{(p)}(t)=d_{i-1}^{(p-1)} \int_{0}^{t} B_{i-1}^{(p-1)}(s) \mathrm{d} s-d_{i}^{(p-1)} \int_{0}^{t} B_{i}^{(p-1)}(s) \mathrm{d} s, \quad i=1, \ldots, p-1, \\
& B_{p}^{(p)}(t)=d_{p-1}^{(p-1)} \int_{0}^{t} B_{p-1}^{(p-1)}(s) \mathrm{d} s
\end{aligned}
$$

where $^{3}$

$$
d_{i}^{(p)}:=\frac{1}{\int_{0}^{1} B_{i}^{(p)}(s) \mathrm{d} s}=p+1 .
$$

Example 2. We have for $p \geq 1$

$$
t=\sum_{i=0}^{p} \frac{i}{p} B_{i}^{(p)}(t) .
$$

The values $\xi_{i, p}^{*}:=\frac{i}{p}$ are called Greville abscissas ${ }^{4}$.

Relation (12) can be easily seen by induction on $p$. The relation is obvious for $p=1$. Assume it is true for $p$, then by degree elevation (5) we get

$$
\begin{aligned}
t & =\sum_{i=0}^{p} \frac{i}{p} B_{i}^{(p)}(t)=\sum_{i=0}^{p} \frac{i}{p}\left(\frac{i+1}{p+1} B_{i+1}^{(p+1)}(t)+\frac{p+1-i}{p+1} B_{i}^{(p+1)}(t)\right) \\
& =B_{p+1}^{(p+1)}(t)+\sum_{i=1}^{p}\left(\frac{i}{p} \frac{p+1-i}{p+1}+\frac{i-1}{p} \frac{i}{p+1}\right) B_{i}^{(p+1)}(t)=\sum_{i=0}^{p+1} \frac{i}{p+1} B_{i}^{(p+1)}(t) .
\end{aligned}
$$

\subsubsection{Geometrical and Graphical Properties}

The algebraic properties listed before have a number of fundamental geometric consequences. Let $\mathbf{P}_{i} \in \mathbb{R}^{d}, i=0, \ldots, p$, be given. The parametric curve

$$
\mathcal{C}(t):=\sum_{i=0}^{p} \mathbf{P}_{i} B_{i}^{(p)}(t)
$$

is called a Bézier curve ${ }^{5}$ in $\mathbb{R}^{d}$. The points $\mathbf{P}_{i}$ are the control points of $\mathcal{C}$, and the polygon they form is referred to as the control polygon of $\mathcal{C}$. Figure 2 shows a quintic Bézier curve in $\mathbb{R}^{3}$ and its control polygon.

\footnotetext{
${ }^{3}$ We introduce this notation for the sake of symmetry with a recurrence relation we need later on in Sect. 4.1.

${ }^{4}$ Referring to Footnote 1, Sergei N. Bernstein used the Greville abscissas in his proof of the Weierstrass theorem [2].

${ }^{5}$ Pierre Bézier was a French engineer working at Renault. His software UNISURF was one of the first CAD systems $[3,40]$.
} 
The graph of any polynomial $q(t)=\sum_{i=0}^{p} q_{i} B_{i}^{(p)}(t)$ of degree $p$ can be seen as a planar Bézier curve by using the Greville abscissas (see Example 2):

$$
\left(\begin{array}{c}
t \\
q(t)
\end{array}\right)=\sum_{i=0}^{p}\left(\begin{array}{c}
\xi_{i, p}^{*} \\
q_{i}
\end{array}\right) B_{i}^{(p)}(t)
$$

and the polygonal line connecting $\left(\begin{array}{c}\xi_{i, p}^{*} \\ q_{i}\end{array}\right), i=0, \ldots, p$, is the control polygon of $q$.

Now we detail the most interesting geometric properties of Bézier curves.

- Affine invariance. From the partition of unity it follows

$$
\mathbf{P}_{i} \in \mathbb{R}^{d}, \mathcal{C}(t)=\sum_{i=0}^{p} \mathbf{P}_{i} B_{i}^{(p)}(t) \quad \Rightarrow \quad A \mathcal{C}(t)+\mathbf{Q}=\sum_{i=0}^{p}\left(A \mathbf{P}_{i}+\mathbf{Q}\right) B_{i}^{(p)}(t)
$$

This means that applying an affine transformation to a Bézier curve is equivalent to applying the same transformation to its control points.

- Convex hull property. The positivity and partition of unity imply, see Fig. 3,

$$
\mathcal{C}(t)=\sum_{i=0}^{p} \mathbf{P}_{i} B_{i}^{(p)}(t) \in \mathcal{H}\left(\mathbf{P}_{0}, \ldots, \mathbf{P}_{p}\right), \quad t \in[0,1]
$$

where $\mathcal{H}\left(\mathbf{P}_{0}, \ldots, \mathbf{P}_{p}\right)$ denotes the convex hull of the points $\mathbf{P}_{0}, \ldots, \mathbf{P}_{p}$.

Fig. 3 Convex hull property for a cubic planar curve.

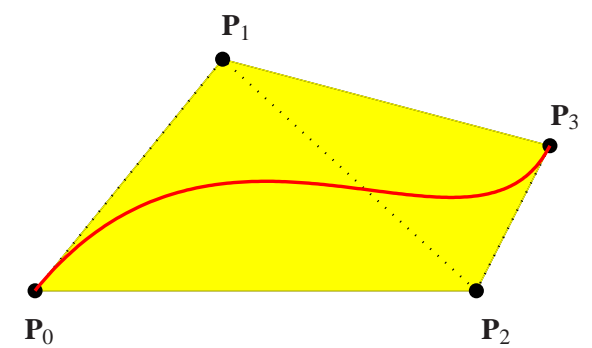

- de Casteljau algorithm. The recurrence relation (4) leads to a stable evaluation algorithm:

$$
\begin{aligned}
\mathcal{C}(t) & =\sum_{i=0}^{p} \mathbf{P}_{i}^{[p]} B_{i}^{(p)}(t) \\
& =\sum_{i=0}^{p} \mathbf{P}_{i}^{[p]}\left[(1-t) B_{i}^{(p-1)}(t)+t B_{i-1}^{(p-1)}(t)\right]=\sum_{i=0}^{p-1} \mathbf{P}_{i}^{[p-1]} B_{i}^{(p-1)}(t)=\ldots=\mathbf{P}_{0}^{[0]}
\end{aligned}
$$

with

$$
\mathbf{P}_{i}^{[k-1]}:=(1-t) \mathbf{P}_{i}^{[k]}+t \mathbf{P}_{i+1}^{[k]}, \quad i=0, \ldots, k-1 .
$$




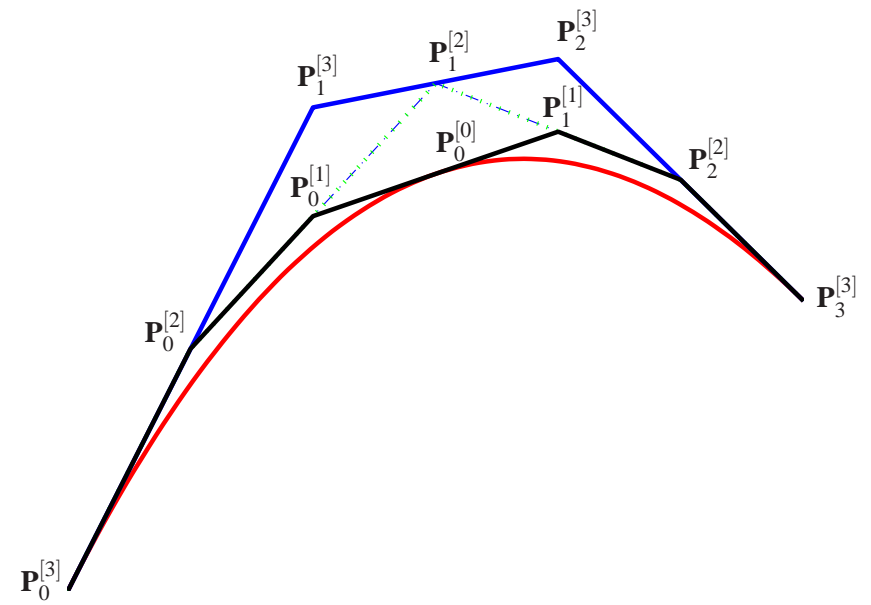

Fig. 4 The de Casteljau algorithm for a cubic Bézier curve.

The strategy (15) allows us to evaluate the curve $\mathcal{C}$ at a fixed parameter value $t$ starting from the control points $\mathbf{P}_{i}^{[p]}$ by using successive linear combinations. Moreover, if $t \in[0,1]$ then we deal with convex combinations which make the evaluation numerically stable, see also Fig. 4.

The previous algorithm is known as the de Casteljau algorithm ${ }^{6}$ and provides a very elegant and stable tool to evaluate a Bézier curve.

- Degree elevation. By degree raising of the Bernstein polynomials (5), and by setting $\mathbf{P}_{-1}:=0, \mathbf{P}_{p+1}:=0$, we have

$$
\sum_{i=0}^{p} \mathbf{P}_{i} B_{i}^{(p)}(t)=\sum_{i=0}^{p+1} \hat{\mathbf{P}}_{i} B_{i}^{(p+1)}(t), \quad \hat{\mathbf{P}}_{i}:=\frac{p+1-i}{p+1} \mathbf{P}_{i}+\frac{i}{p+1} \mathbf{P}_{i-1} .
$$

Any Bézier curve of degree $p$ can be seen as a Bézier curve of degree $p+1$, and the new control points can be obtained from the previous ones by convex combinations, see (16). In other words, the new control polygon is obtained from the previous one by "cutting some corners", see also Fig. 5, so that it is closer to the curve.

- Derivatives of a Bézier curve. Applying (6) inductively we obtain:

$$
\frac{\mathrm{d} \mathcal{C}(t)}{\mathrm{d} t}=p \sum_{i=0}^{p-1}\left(\mathbf{P}_{i+1}-\mathbf{P}_{i}\right) B_{i}^{(p-1)}(t), \quad p \geq 1,
$$

\footnotetext{
${ }^{6}$ Paul de Casteljau is a French physicist and mathematician. He worked at Citroën, where he developed his famous algorithm for evaluation of a family of polynomial curves [5, 15]. The same curves were used independently by Pierre Bézier at Renault. The connection between these curves and Bernstein polynomials was not clear at the beginning.
} 


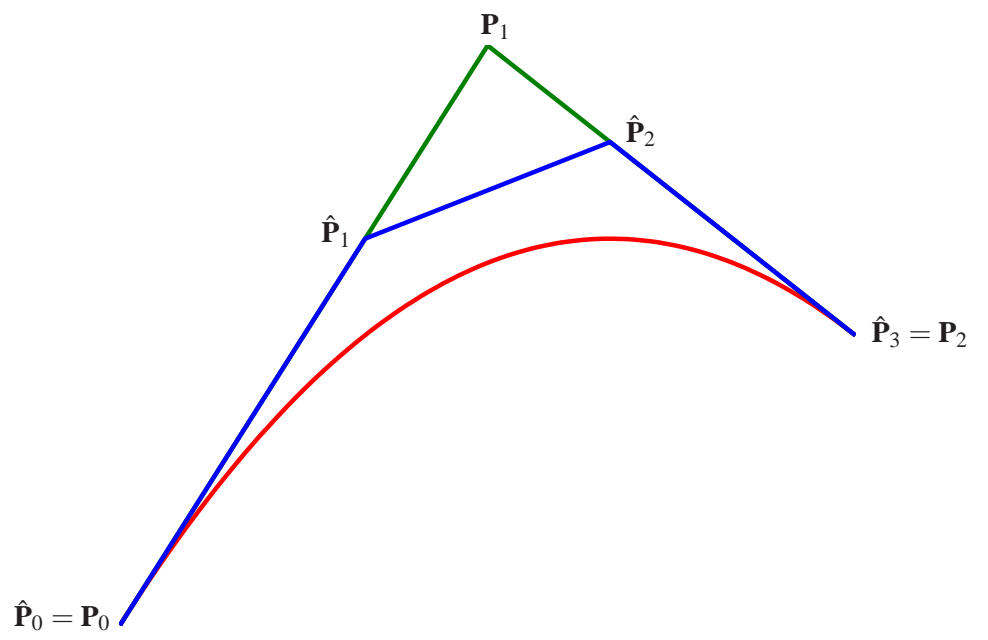

Fig. 5 Degree elevation for a quadratic Bézier curve.

and

$$
\frac{\mathrm{d}^{r} \mathcal{C}(t)}{\mathrm{d} t^{r}}=\frac{p !}{(p-r) !} \sum_{i=0}^{p-r} \Delta_{r}\left(\mathbf{P}_{i}\right) B_{i}^{(p-r)}(t), \quad p \geq r
$$

with

$$
\Delta_{r}\left(\mathbf{P}_{i}\right):=\sum_{k=0}^{r}\left(\begin{array}{l}
r \\
k
\end{array}\right)(-1)^{r-k} \mathbf{P}_{i+k}
$$

Only $r+1$ control points are involved in the expression for the $r$-th order derivative evaluated at the two end points. Moreover, (17) implies that the control polygon is tangent to the curve at both end points.

- Smooth joints of Bézier curves. $C^{r}$ continuity of two adjacent Bézier curves

$$
\sum_{j=0}^{p} \mathbf{P}_{j}^{L} B_{j}^{(p)}(t), \quad \sum_{j=0}^{p} \mathbf{P}_{j}^{R} B_{j}^{(p)}(t)
$$

has a simple geometric interpretation thanks to the local behavior of the derivatives at the end points, see (17) and (18). In particular, $C^{0}$ continuity just requires that $\mathbf{P}_{p}^{L}=\mathbf{P}_{0}^{R}$, while $C^{1}$ continuity implies, in addition, that the two segments $\mathbf{P}_{p-1}^{L} \mathbf{P}_{p}^{L}$ and $\mathbf{P}_{0}^{R} \mathbf{P}_{1}^{R}$ are collinear, see Fig. 6 .

- Variation diminishing. Let us consider a polynomial in Bernstein form

$$
q(t)=\sum_{i=0}^{p} q_{i} B_{i}^{(p)}(t), \quad t \in[0,1] .
$$




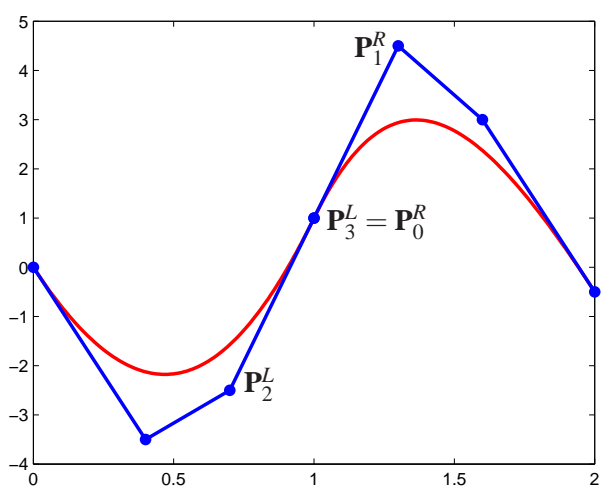

Fig. $6 C^{1}$ joint between two cubic Bézier curves.

From the derivative formulas (17)-(18) we have

$$
\begin{aligned}
\frac{\mathrm{d} q(t)}{\mathrm{d} t} & =p \sum_{i=0}^{p-1}\left(q_{i+1}-q_{i}\right) B_{i}^{(p-1)}(t), \quad p \geq 1, \\
\frac{\mathrm{d}^{2} q(t)}{\mathrm{d} t^{2}} & =p(p-1) \sum_{i=0}^{p-2}\left(q_{i+2}-2 q_{i+1}+q_{i}\right) B_{i}^{(p-2)}(t), \quad p \geq 2 .
\end{aligned}
$$

By taking into account the positivity of the Bernstein polynomials, it follows that the polynomial $q$ inherits the same shape behavior as its control polygon. More precisely, $q$ is positive if its coefficients $q_{i}$ are positive, $q$ is increasing if its coefficients form an increasing sequence, and $q$ is convex if its control polygon is convex ${ }^{7}$.

These properties are usually referred to as variation diminishing properties, and they can be suitably extended to the curve case, see the next subsection.

\subsection{Total Positivity and Optimal Bases}

In this subsection we show that the variation diminishing properties of the Bernstein representation mentioned above are a consequence of a fundamental property of Bernstein polynomials, namely total positivity. Moreover, we show that Bernstein polynomials are in a suitable sense the best basis to represent polynomials.

\footnotetext{
${ }^{7}$ It follows that the Bernstein operator $B_{p}(f,$.$) , as defined in Footnote 1, is shape preserving in the$ sense that if $f$ is positive and/or monotone and/or convex then $B_{p}(f,$.$) is positive and/or monotone$ and/or convex as well.
} 

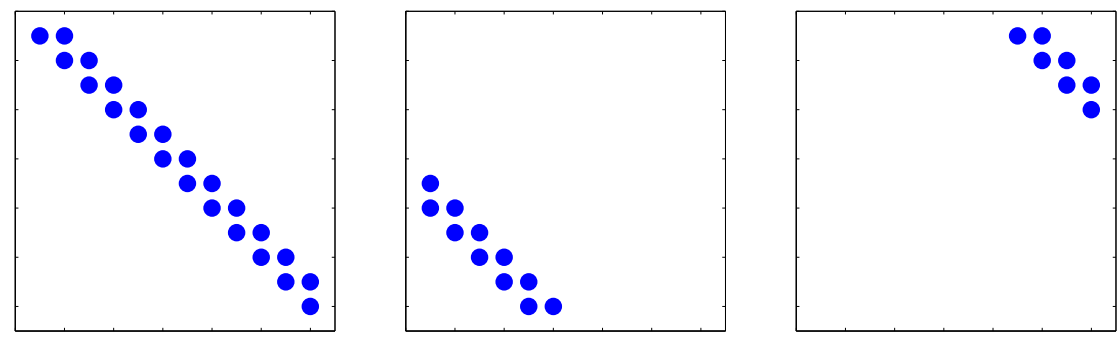

Fig. 7 Bidiagonal matrices.

\subsubsection{Total Positivity}

Here we introduce totally positive matrices and some of their properties of salient interest in connection with features of bases of vector spaces [29, 35].

Definition 2. A matrix $A \in \mathbb{R}^{(n, m)}$ is totally positive (TP) if the determinant of any submatrix is positive, i.e. for $k=1, \ldots, \min (n, m)$

$$
\operatorname{det}\left(\begin{array}{ccc}
a_{\alpha_{1}, \beta_{1}} & \ldots & a_{\alpha_{1}, \beta_{k}} \\
\vdots & & \vdots \\
a_{\alpha_{k}, \beta_{1}} & \ldots & a_{\alpha_{k}, \beta_{k}}
\end{array}\right) \geq 0, \quad 1 \leq \alpha_{1}<\ldots<\alpha_{k} \leq n, \quad 1 \leq \beta_{1}<\ldots<\beta_{k} \leq m .
$$

Moreover, we say that A is strictly totally positive (STP) if (19) is strictly positive.

Definition 3. A matrix is stochastic if it is positive and the entries in each row sum up to one.

For STP matrices the set of submatrices to be checked can be reduced, see [1, Theorem 2.5] and [28].

Lemma 1 (Fekete, 1912). A matrix is STP if and only if any square submatrix consisting of consecutive rows/columns is STP.

Finally, we introduce the general concept of bidiagonal matrices, see Fig. 7.

Definition 4. A matrix A is bidiagonal if $a_{i, j} \neq 0$ implies $l \leq j-i \leq l+1$ for some $l$.

It is not difficult to prove the following result.

Theorem 1. Any positive bidiagonal matrix is TP.

The Cauchy-Binet theorem, see [1, Sect. 1], leads to the following result.

Theorem 2. The product of (stochastic) TP matrices is a (stochastic) TP matrix.

The main properties of (stochastic) TP matrices are a consequence of the following fundamental result, see [31, Theorems 2.2 and 2.3]. 
Theorem 3. A matrix is (stochastic) TP if and only if it is the product of (stochastic) positive bidiagonal matrices.

The factorization in Theorem 3 implies that for a TP matrix $A$ and any vector $\mathbf{v}$

$$
S^{-}(A \mathbf{v}) \leq S^{-}(\mathbf{v})
$$

where $S^{-}(\mathbf{w})$ denotes the number of (strict) changes in sign in the components of w. Indeed, thanks to Theorem 3 it is sufficient to prove (20) for positive bidiagonal matrices.

Now we connect TP matrices with bases of vector spaces.

Definition 5. A basis $\left\{\varphi_{0}, \ldots, \varphi_{p}\right\}$ of a space $\mathbb{U}_{p}$ is totally positive on an interval $I \subset \mathbb{R}$ if any collocation matrix

$$
\left(\begin{array}{ccc}
\varphi_{0}\left(t_{1}\right) & \ldots & \varphi_{p}\left(t_{1}\right) \\
\vdots & & \vdots \\
\varphi_{0}\left(t_{r}\right) & \ldots & \varphi_{p}\left(t_{r}\right)
\end{array}\right)
$$

is TP, where

$$
t_{1}<t_{2}<\ldots<t_{r}, \quad t_{i} \in I, i=1, \ldots, r .
$$

Moreover, the basis is normalized if $\sum_{j=0}^{p} \varphi_{j}=1$.

By using Theorem 2, we obtain the following proposition.

Proposition 1. Let $\left\{\varphi_{0}, \ldots, \varphi_{p}\right\}$ be TP on I.

- if $f: J \rightarrow I$ is increasing then $\left\{\varphi_{0} \circ f, \ldots, \varphi_{p} \circ f\right\}$ is TP on $J$;

- if $g$ is positive on I then $\left\{g \varphi_{0}, \ldots, g \varphi_{p}\right\}$ is TP on I;

- if $A:=\left(a_{i j}\right)$ is a TP matrix then $\left\{\sum_{j=0}^{p} a_{0 j} \varphi_{j}, \ldots, \sum_{j=0}^{p} a_{p j} \varphi_{j}\right\}$ is TP on I.

We note that evaluating $\sum_{i=0}^{p} c_{i} \varphi_{i}$ at any sequence of points $t_{1}<t_{2}<\ldots<t_{r}$, $t_{i} \in I, i=1, \ldots, r$, is nothing else than multiplying the collocation matrix (21) by the vector of the coefficients $\left(c_{0}, \ldots, c_{p}\right)^{T}$. This immediately leads to the following result.

Corollary 1. If $\left\{\varphi_{0}, \ldots, \varphi_{p}\right\}$ is a TP basis of $\mathbb{U}_{p}$ and $c_{0}, \ldots, c_{p} \in \mathbb{R}$, then the number of sign changes of the element $\left(\sum_{i=0}^{p} c_{i} \varphi_{i}\right) \in \mathbb{U}_{p}$ is less than or equal to the number of sign changes of $\left(c_{0}, \ldots, c_{p}\right)$.

Let $\mathbf{C}_{j} \in \mathbb{R}^{d}$ be given, then

$$
\mathcal{C}(t):=\sum_{j=0}^{p} \mathbf{C}_{j} \varphi_{j}(t), \quad t \in I
$$

defines a curve in $\mathbb{R}^{d}$ with components in $\mathbb{U}_{p}$. Generalizing the notation used for Bernstein polynomials, we say that $\mathbf{C}_{0}, \ldots, \mathbf{C}_{p}$ are the control points of $\mathcal{C}$ (with respect to the basis $\left.\left\{\varphi_{0}, \ldots, \varphi_{p}\right\}\right)$ and the polygonal line they form is the control polygon of $\mathcal{C}$. 
A more geometric consequence of the factorization given by Theorem 3 is the following.

Proposition 2 (Variation diminishing). Let $\left\{\varphi_{0}, \ldots, \varphi_{p}\right\}$ be a normalized TP basis. Define the planar curve $\mathcal{C}(t)=\sum_{j=0}^{p} \mathbf{C}_{j} \varphi_{j}(t), t \in I, \mathbf{C}_{j} \in \mathbb{R}^{2}$. Then the number of times $\mathcal{C}$ crosses any straight line $\boldsymbol{\ell}$ is bounded by the number of times its control polygon crosses $\ell$.

Proof. Let $a x+b y+c=0$ be the equation of $\boldsymbol{\ell}$ and let us put $\mathbf{C}_{j}:=\left(C_{j, x}, C_{j, y}\right)^{T}$, and $\mathcal{C}(t):=\left(\mathcal{C}_{x}(t), \mathcal{C}_{y}(t)\right)^{T}$. By Corollary 1 , if the basis is normalized and TP, the number of changes in sign in

$$
a \mathcal{C}_{x}(t)+b \mathcal{C}_{y}(t)+c=\sum_{j=0}^{p}\left(a C_{j, x}+b C_{j, y}+c\right) \varphi_{j}(t)
$$

is bounded above by the number of changes in sign in the sequence

$$
a C_{j, x}+b C_{j, y}+c, j=0, \ldots, p .
$$

Of course, any change in sign in the sequence above corresponds to a cross of the control polygon and the straight line $\ell$.

The previous result ensures that TP bases provide shape-preserving representations, see Fig. 8. As an example, if the control polygon is convex the corresponding curve is convex as well.

Fig. 8 Variation diminishing.

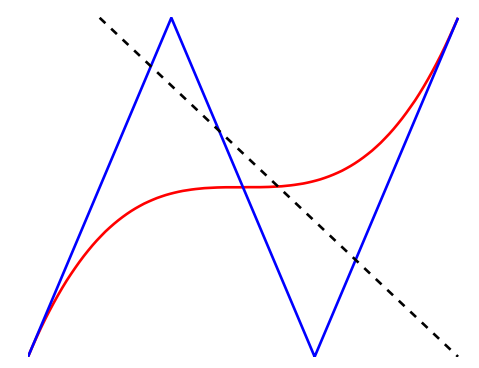

Now we provide some relevant examples of TP bases for the space $\mathbb{P}_{p}$.

Example 3. The monomial basis $\left\{1, t, \ldots, t^{p}\right\}$ is TP on $[0,+\infty)$. This can be easily seen as follows. By using the relation

$$
\operatorname{det}\left(\begin{array}{cccc}
1 & t_{1} & \ldots & t_{1}^{k} \\
\vdots & \vdots & & \vdots \\
1 & t_{k+1} & \ldots & t_{k+1}^{k}
\end{array}\right)=\prod_{1 \leq i<j \leq k+1}\left(t_{j}-t_{i}\right),
$$

we may conclude from the Fekete lemma that any collocation matrix is STP on $(0,+\infty)$. Then, by continuity, any collocation matrix is TP on $[0,+\infty)$. 
Example 4. Let us consider Proposition 1 with the monomial basis $\varphi_{i}(t):=t^{i}, i=$ $0, \ldots, p$

$$
f:[0,1) \rightarrow[0,+\infty), f(t):=\frac{t}{1-t}
$$

and

$$
g(t):=(1-t)^{p}
$$

It follows that

$$
g(t)\left[\varphi_{i} \circ f\right](t)=(1-t)^{p}\left(\frac{t}{1-t}\right)^{i} .
$$

Since we know from the previous example that the monomial basis is TP on $[0,+\infty)$, we have that

$$
t^{i}(1-t)^{p-i}, i=0, \ldots, p
$$

is a TP basis for $\mathbb{P}_{p}$ on $[0,1)$. By noting that the diagonal matrix with diagonal entries $\left(\begin{array}{l}p \\ i\end{array}\right)$ is TP, and by applying again Proposition 1, we obtain that the Bernstein polynomials are a TP basis on $[0,1)$. By continuity, the TP property extends to $[0,1]$.

\subsubsection{Optimal Bases}

In this subsection we prove that Bernstein polynomials have an optimality property that makes them the best basis for $\mathbb{P}_{p}$ from the geometric point of view. To this aim we give the following result.

Theorem 4 ([14], Theorem 2.2). Suppose that the space $\mathbb{U}_{p}$ possesses a normalized TP $(N T P)$ basis. Then it possesses a unique NTP basis $\left(\vartheta_{0}, \ldots, \vartheta_{p}\right)$ such that any other NTP basis $\left(\varphi_{0}, \ldots, \varphi_{p}\right)$ is given by

$$
\left(\varphi_{0}, \ldots, \varphi_{p}\right)=\left(\vartheta_{0}, \ldots, \vartheta_{p}\right) K
$$

where the matrix $K$ is stochastic and TP. We then say that $\left(\vartheta_{0}, \ldots, \vartheta_{p}\right)$ is the optimal NTP basis (in short, the ONTP basis) of the space $\mathbb{U}_{p}$.

The matrix $K$ in (22) is nonsingular and stochastic because the two involved bases are normalized. Therefore, the following theorem is of interest $[30,51]$.

Theorem 5. A nonsingular stochastic TP matrix can be decomposed as the product of matrices of the form

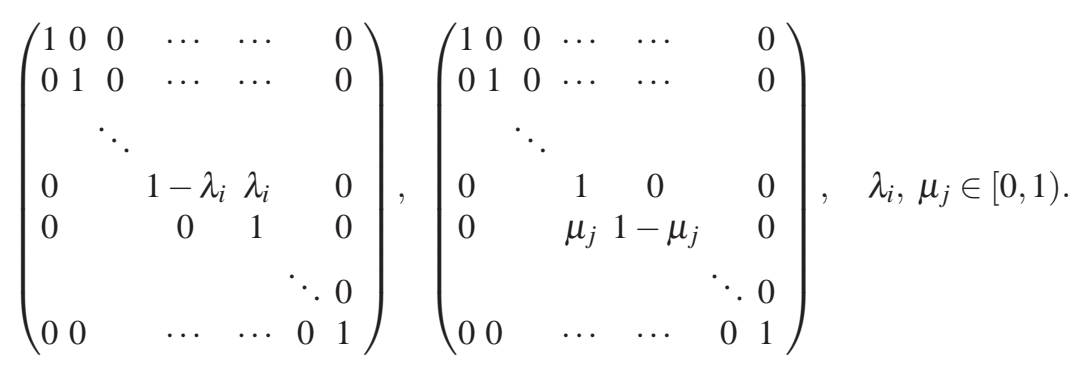



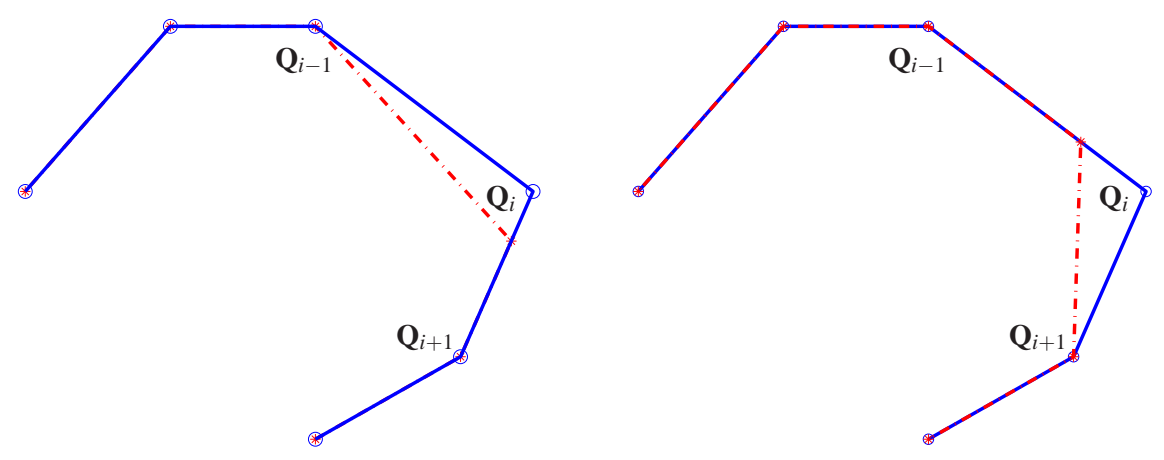

Fig. 9 Elementary corner cuttings.

We say that each matrix in (23) describes an elementary corner cutting. This is motivated by the fact that, see also Fig. 9 ,

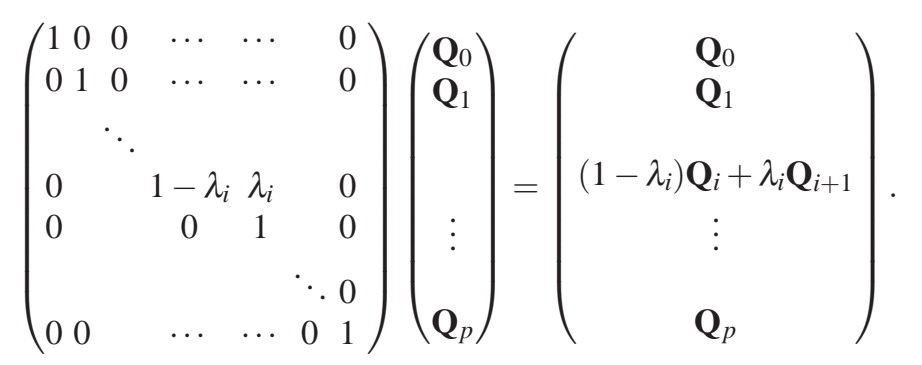

Let $\left\{\vartheta_{0}, \ldots, \vartheta_{p}\right\}$ be the ONTP basis for $\mathbb{U}_{p}$, and $\left\{\varphi_{0}, \ldots, \varphi_{p}\right\}$ be an NTP basis. Then

$$
\mathcal{C}(t):=\sum_{j=0}^{p} \mathbf{Q}_{j} \varphi_{j}(t)=\sum_{j=0}^{p} \mathbf{Q}_{j} \sum_{i=0}^{p} k_{i j} \vartheta_{i}(t)=\sum_{i=0}^{p}\left(\sum_{j=0}^{p} k_{i j} \mathbf{Q}_{j}\right) \vartheta_{i}(t)=\sum_{i=0}^{p} \mathbf{P}_{i} \vartheta_{i}(t)
$$

where

$$
\left(\begin{array}{c}
\mathbf{P}_{0} \\
\vdots \\
\mathbf{P}_{p}
\end{array}\right)=K\left(\begin{array}{c}
\mathbf{Q}_{0} \\
\vdots \\
\mathbf{Q}_{p}
\end{array}\right)
$$

It follows that the control polygon of $\mathcal{C}$ with respect to the ONTP basis is obtained by successive elementary corner cuttings from the control polygon with respect to any other NTP basis. Hence, the control polygon with respect to the ONTP basis is the closest one to $\mathcal{C}$, see also Fig. 10. It lies "between" $\mathcal{C}$ and the control polygon with respect to any other NTP basis. As a result, the coefficients of an element of $\mathbb{U}_{p}$ with respect to the ONTP basis provide the most accurate description from a geometric point view of the element itself. ONTP bases can be characterized in 

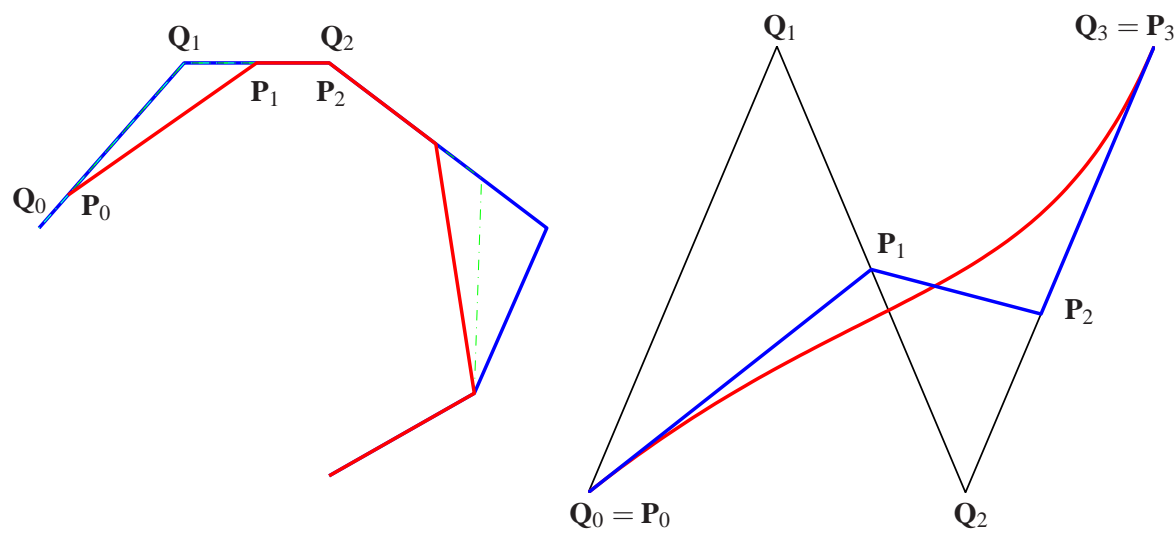

Fig. 10 Left: corner cutting as successive elementary corner cuttings. Right: control polygons with respect to an NTP basis (black) and to the ONTP basis (blue) for the same curve (red).

terms of the behavior of the basis elements at the two ends of their support (with respect to $I$ ) as stated in the following theorem.

Theorem 6 ([51], Proposition 3.2). Suppose that the space $\mathbb{U}_{p}$ possesses an NTP basis on I, and denote by $\left(\vartheta_{0}, \ldots, \vartheta_{p}\right)$ the ONTP basis. Then,

- for $i=0, \ldots, p$, the set $I_{i}:=\left\{x \in I: \vartheta_{i}(x) \neq 0\right\}$ is an interval;

- setting $a_{i}:=\inf I_{i}, b_{i}:=\sup I_{i}$, for $0 \leq i \leq p$, we have, for $k<i$,

$$
a_{k} \leq a_{i}, b_{k} \leq b_{i}, \quad \lim _{x \rightarrow a_{k}^{+}} \frac{\vartheta_{i}(x)}{\vartheta_{k}(x)}=0, \lim _{x \rightarrow b_{i}^{-}} \frac{\vartheta_{k}(x)}{\vartheta_{i}(x)}=0 .
$$

Example 5. If $\mathbb{U}_{p}=\mathbb{P}_{p}$ and $\left(\vartheta_{0}, \ldots, \vartheta_{p}\right)$ are the Bernstein polynomials, we have $I_{i}=[0,1], i=0, \ldots, p$ in the previous theorem. Then, from Example 4 and from (7)-(8) we immediately obtain that Bernstein polynomials are the ONTP basis of $\mathbb{P}_{p}$ on $[0,1]$. Therefore, Bernstein polynomials are the optimal way to represent any polynomial of degree $p$, see also [12].

Remark 1. It turns out that, besides the above optimality property, Bernstein polynomials also possess optimal numerical stability properties. We refer to [27] for further details.

\section{Piecewise Spaces: B-Splines}

In this section we focus on the most powerful tool in CAGD: B-spline curves and surfaces. We present the definition and main properties of the B-spline basis as well 
as the properties of the space they span. Analogously to the case of Bernstein polynomials, B-splines form the best basis for the space of piecewise polynomials. The results that we present and their formulation are basically taken from [8], and the most part of the pictures are generated with the corresponding MATLAB ${ }^{\circledR}$ Toolbox developed by the same author. The main difference with [8] is that we detail all the results for a finite sequence of knots. This choice is surely less elegant and requires sometimes a heavier notation, but it is more suitable from the application point of view.

Finally, we briefly describe the tensor-product extension to the multivariate case, and the rational form NURBS.

\subsection{Definition and Main Properties}

In this subsection we define $B$-splines ${ }^{8}$ and we derive some of their main properties. Let a sequence of knots be given

$$
\Xi:=\left\{\xi_{1} \leq \xi_{2} \leq \ldots \leq \xi_{n+p+1}\right\}, \quad n, p \in \mathbb{N},
$$

we say that the knot $\xi_{i}$ has multiplicity $1 \leq \rho_{i} \leq p+1$ if

$$
\ldots<\xi_{i}=\xi_{i+1}=\ldots=\xi_{i+\rho_{i}-1}<\xi_{i+\rho_{i}} .
$$

Usually, one takes at the end points a multiplicity $p+1$, i.e.,

$$
\xi_{1}=\ldots=\xi_{p+1}<\ldots<\xi_{n+1}=\ldots=\xi_{n+p+1} .
$$

Definition 6. The B-splines $B_{i, \Xi}^{(p)}$ of degree p, related to the knots $\Xi$, are given by

$$
\begin{aligned}
B_{i, \Xi}^{(0)}(t) & := \begin{cases}1, & \text { if } t \in\left[\xi_{i}, \xi_{i+1}\right) \\
0, & \text { elsewhere, }\end{cases} \\
B_{i, \Xi}^{(p)}(t) & :=\frac{t-\xi_{i}}{\xi_{i+p}-\xi_{i}} B_{i, \Xi}^{(p-1)}(t)+\frac{\xi_{i+p+1}-t}{\xi_{i+p+1}-\xi_{i+1}} B_{i+1, \Xi}^{(p-1)}(t), \quad p \geq 1,
\end{aligned}
$$

where fractions with zero denominator are considered to be zero.

The knot sequence (25) allows us to define $n$ B-splines of degree $p$, namely $B_{1, \Xi}^{(p)}, \ldots, B_{n, \Xi}^{(p)}$. Figure 11 illustrates some B-splines of different degree.

\footnotetext{
${ }^{8}$ B-splines were introduced by Curry and Schoenberg [20] as divided differences of truncated powers, see e.g. [8, Chapter IX]. Here we present an equivalent definition in terms of a recurrence relation. The original definition can be used to prove properties of the B-splines, but it is not suited for numerical evaluation, see [6]. On the other hand, Definition 6 only uses convex combinations which ensure a numerically stable procedure. Nevertheless, Isaac J. Schoenberg is indisputably considered as the father of splines although other authors considered this concept earlier, see e.g. $[11,36,58]$.
} 

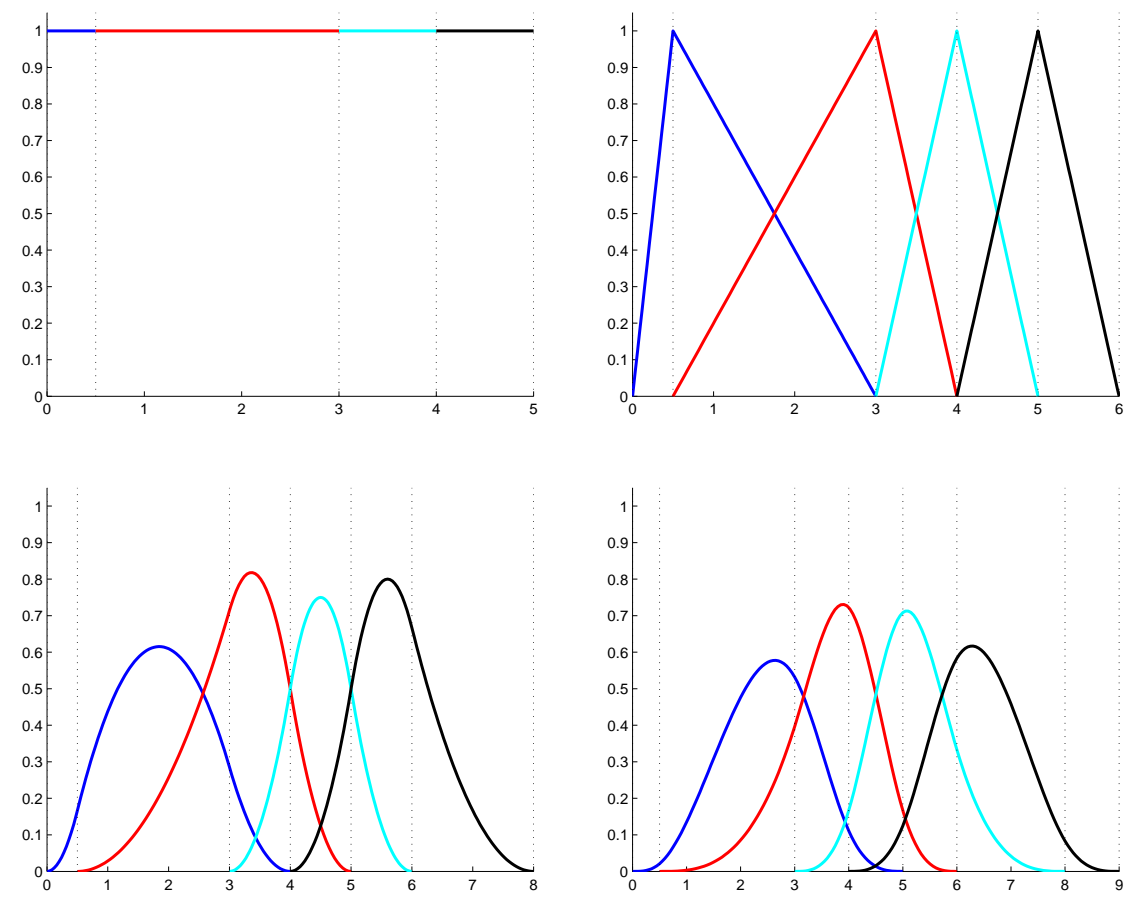

Fig. 11 B-splines of degree 0, 1, 2 and 3.

By setting

$$
\omega_{i, p}(t):=\frac{t-\xi_{i}}{\xi_{i+p}-\xi_{i}}
$$

we have

$$
B_{i, \Xi}^{(p)}(t)=\omega_{i, p}(t) B_{i, \Xi}^{(p-1)}(t)+\left(1-\omega_{i+1, p}(t)\right) B_{i+1, \Xi}^{(p-1)}(t),
$$

and by repeating the recurrence relation (30) we obtain

$$
\begin{aligned}
B_{i, \Xi}^{(p)}(t)=\omega_{i, p} & (t) \omega_{i, p-1}(t) B_{i, \Xi}^{(p-2)}(t)+\omega_{i, p}(t)\left(1-\omega_{i+1, p-1}(t)\right) B_{i+1, \Xi}^{(p-2)}(t) \\
& +\left(1-\omega_{i+1, p}(t)\right) \omega_{i+1, p-1}(t) B_{i+1, \Xi}^{(p-2)}(t) \\
& +\left(1-\omega_{i+1, p}(t)\right)\left(1-\omega_{i+2, p-1}(t)\right) B_{i+2, \Xi}^{(p-2)}(t) \\
=\ldots & =\omega_{i, p}(t) \omega_{i, p-1}(t) \ldots \omega_{i, 1}(t) B_{i, \Xi}^{(0)}(t)+\ldots \\
& +\left(1-\omega_{i+1, p}(t)\right)\left(1-\omega_{i+2, p-1}(t)\right) \ldots\left(1-\omega_{i+p, 1}(t)\right) B_{i+p, \Xi}^{(0)}(t) .
\end{aligned}
$$

We note that each factor in the coefficient of $B_{i+j, \Xi}^{(0)}$ in the previous expansion is a polynomial of degree 1 with respect to $t$, which is positive in $\left[t_{i+j}, t_{i+j+1}\right)$. Hence, 

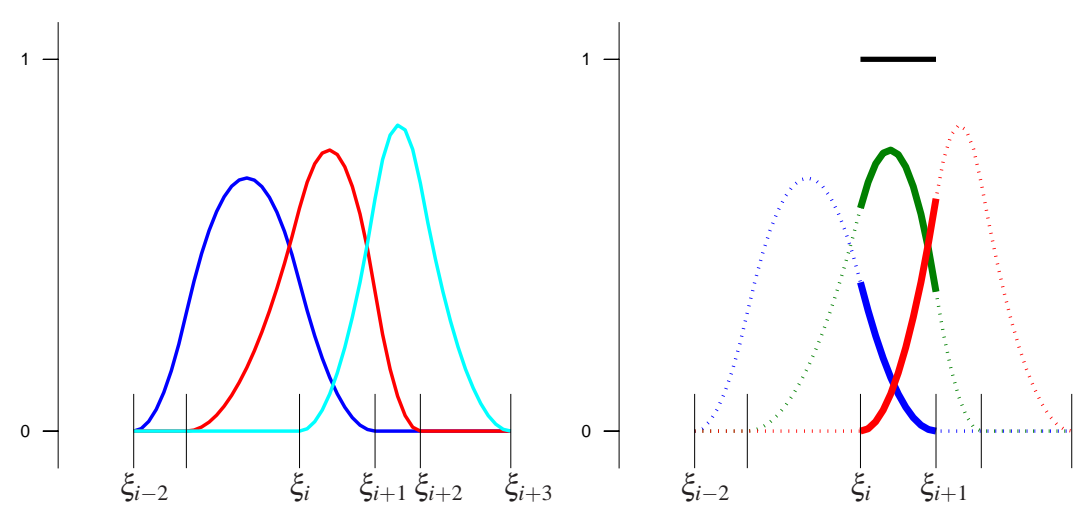

Fig. 12 B-splines of degree 2. Left: compact support. Right: local partition of unity.

from (28) we immediately obtain the following fundamental properties, see also Fig. 11.

- Piecewise polynomials: $B_{i, \Xi}^{(p)}(t) \in \mathbb{P}_{p}, t \in\left[\xi_{j}, \xi_{j+1}\right)$.

- Positivity: $B_{i, \Xi}^{(p)} \geq 0$, and $B_{i, \Xi}^{(p)}(t)>0, t \in\left(\xi_{i}, \xi_{i+p+1}\right)$.

- Compact support ${ }^{9}$ :

$$
\begin{array}{ll}
B_{i, \Xi}^{(p)}(t)=0, & t \notin\left[\xi_{i}, \xi_{i+p+1}\right], \\
B_{i, \Xi}^{(p)}(t)=0, & t \in\left[\xi_{r}, \xi_{r+1}\right), i \notin\{r, r-1, \ldots, r-p\} .
\end{array}
$$

The compact support of some quadratic B-splines is illustrated in Fig. 12 (left).

Remark 2. Let us consider the knot sequence (27). In this case the B-splines $B_{i, \Xi}^{(p)}$, $i=1, \ldots, n$, have their support in the interval $\left[\xi_{p+1}, \xi_{n+1}\right]$. Nevertheless, according to Definition 6, all of them vanish at the right end point $\xi_{n+1}$. In order to avoid this asymmetric behavior at the end points of this interval, we would like to slightly modify our definition and to assume that each B-spline is left-continuous at $\xi_{n+1}$. In this way, we have $B_{n, \Xi}^{(p)}\left(\xi_{n+1}\right)=1$.

Given a general knot sequence (25), the interval $\left[\xi_{p+1}, \xi_{n+1}\right]$ plays a crucial role in the following ${ }^{10}$. Motivated by the considerations in Remark 2 , we slightly modify our definition and we assume from now on that each B-spline of degree $p$ is leftcontinuous at $\xi_{n+1}$ for a general knot sequence (see also [8, Chapter IX]), i.e.,

$$
B_{i, \Xi}^{(p)}\left(\xi_{n+1}\right):=\lim _{t \rightarrow \xi_{n+1}^{-}} B_{i, \Xi}^{(p)}(t) .
$$

${ }^{9}$ The support of $f$ is the closure of the set $\{x: f(x) \neq 0\}$.

${ }^{10}$ This interval is called basic interval in [8, Chapter IX]. 

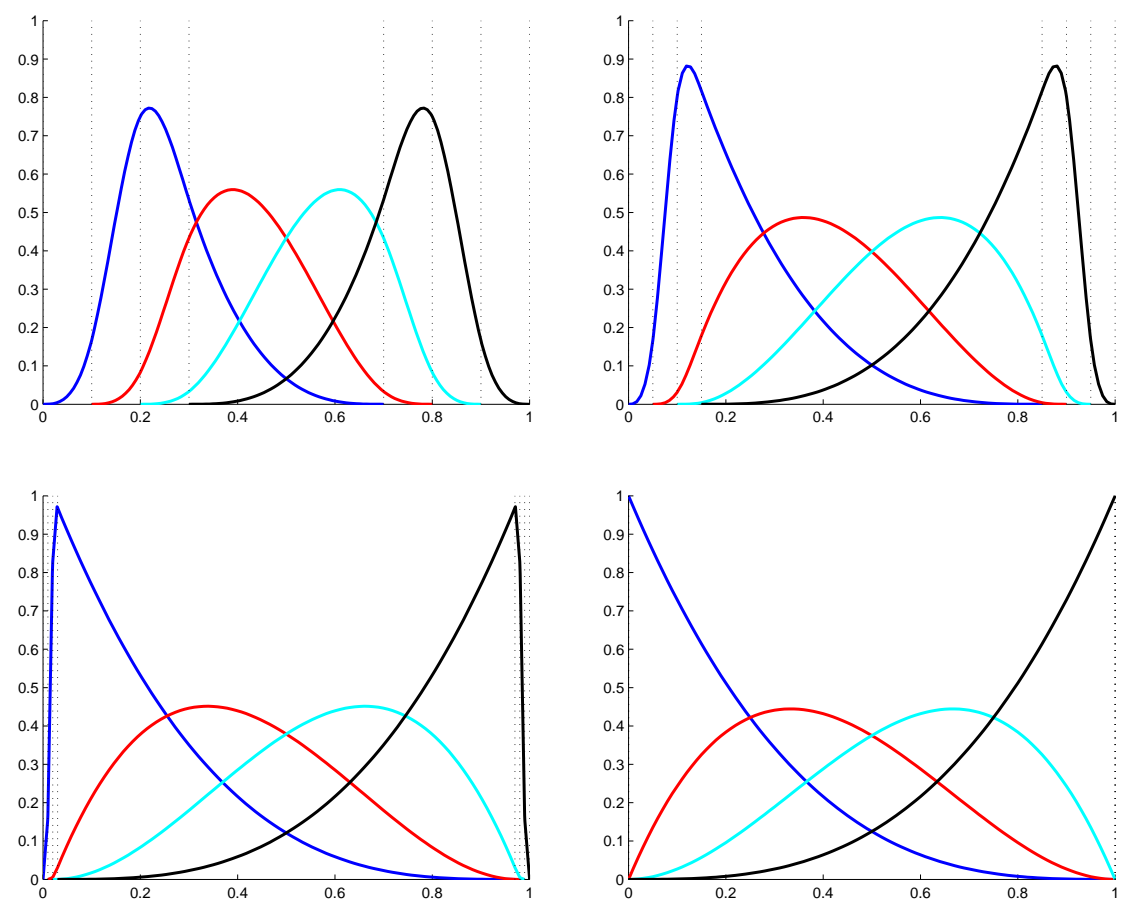

Fig. 13 B-splines of degree 3 over particular sequences of knots. In these pictures, four knots are moving towards the left as well as towards the right end of the interval $[0,1]$.

Example 6. Considering the sequence of knots

$$
\Xi:=\left\{0=\xi_{1}=\ldots=\xi_{p+1}<\xi_{p+2}=\ldots=\xi_{p+p+2}=1\right\},
$$

we get $B_{i, \Xi}^{(p)}(t)=B_{i-1}^{(p)}(t), i=1, \ldots, p+1$, i.e. the Bernstein polynomials, see Fig. 13 .

Definition 7. The spline space $\mathbb{S}_{p, \Xi}$ is the space spanned by the B-splines over the interval $\left[\xi_{p+1}, \xi_{n+1}\right]$, i.e.,

$$
\mathbb{S}_{p, \Xi}:=\left\{\sum_{j=1}^{n} c_{j} B_{j, \Xi}^{(p)}(t), t \in\left[\xi_{p+1}, \xi_{n+1}\right], c_{j} \in \mathbb{R}\right\} .
$$

\subsection{The Space $\mathbb{S}_{p, \Xi}$}

In this subsection we study the properties of the space $\mathbb{S}_{p, \Xi}$. We prove that such a space is nothing else than the space of piecewise polynomials of degree $p$ defined by 
a given sequence of break points and with some prescribed smoothness conditions. The set of knots $\Xi$ must be suitably selected according to the break points and the smoothness conditions. Therefore, the B-splines are a basis of such a space of piecewise polynomials. As we will see later, this basis is the optimal one.

Let

$$
X:=\left\{x_{0}<x_{1}<\ldots<x_{l+1}\right\}, \quad l \in \mathbb{N},
$$

be a set of (strictly increasing) break points, and let $\mathfrak{V}:=\left\{v_{1}, \ldots, v_{l}\right\}$ be a sequence of integers.

Definition 8. The space of piecewise polynomials with smoothness $\mathfrak{V}$ at the break points $X$ is given by

$$
\begin{aligned}
& \mathbb{P}_{p, X}^{\mathfrak{V}}:=\left\{s:\left.s\right|_{\left[x_{i}, x_{i+1}\right)}\right. \in \mathbb{P}_{p}, i=0, \ldots, l, \\
&\left.s^{(j)}\left(x_{i}^{+}\right)=s^{(j)}\left(x_{i}^{-}\right), j=0, \ldots, v_{i}-1, i=1, \ldots, l\right\} .
\end{aligned}
$$

In the previous definition it makes only sense to choose $0 \leq v_{j} \leq p, j=1, \ldots, l$. In this case it is easy to see that

$$
\operatorname{dim}\left(\mathbb{P}_{p, X}^{\mathfrak{V}}\right)=(l+1)(p+1)-\sum_{j=1}^{l} v_{j}=p+1+\sum_{j=1}^{l}\left(p+1-v_{j}\right)
$$

and

$$
\mathbb{P}_{p, X}^{\mathfrak{V}}=\left\langle 1, t, \ldots, t^{p},\left(t-x_{1}\right)_{+}^{v_{1}}, \ldots,\left(t-x_{1}\right)_{+}^{p}, \ldots,\left(t-x_{l}\right)_{+}^{v_{l}}, \ldots,\left(t-x_{l}\right)_{+}^{p}\right\rangle,
$$

where $(t)_{+}^{r}:=(\max (t, 0))^{r}$ denotes the truncated power function.

In the following we will prove that the polynomials of degree $p$ and the truncated power functions in (35) belong to $\mathbb{S}_{p, \Xi}$ for a suitable knot sequence $\Xi$.

\subsubsection{Polynomials in $\mathbb{S}_{p, \Xi}$}

Here we provide the representation of polynomials of degree $p$ in terms of B-splines of the same degree.

Lemma 2. We have

$$
\frac{(t-\tau)^{p-\mu}}{(p-\mu) !}=\sum_{i=1}^{n} \frac{(-1)^{\mu}}{p !} \frac{\mathrm{d}^{\mu} \psi_{i, p}(\tau)}{\mathrm{d} \tau^{\mu}} B_{i, \Xi}^{(p)}(t), \quad t \in\left[\xi_{p+1}, \xi_{n+1}\right], \quad 0 \leq \mu \leq p
$$

where

$$
\psi_{i, 0}(\tau):=1, \quad \psi_{i, p}(\tau):=\left(\xi_{i+1}-\tau\right) \ldots\left(\xi_{i+p}-\tau\right) .
$$

Proof. Note that

$$
\omega_{i, p}(t) \psi_{i, p}(\tau)+\left(1-\omega_{i, p}(t)\right) \psi_{i-1, p}(\tau)=(t-\tau) \psi_{i, p-1}(\tau) .
$$


By taking into account the support of $B_{i, \Xi}^{(k)}, k=0, \ldots, p$, see (31), we obtain that for $t \in\left[\xi_{p+1}, \xi_{n+1}\right)$,

$$
\begin{aligned}
\sum_{i=1}^{n} \psi_{i, p}(\tau) B_{i, \Xi}^{(p)}(t) & =\sum_{i=1}^{n} \psi_{i, p}(\tau)\left[\left(\omega_{i, p}(t) B_{i, \Xi}^{(p-1)}(t)+\left(1-\omega_{i+1, p}(t)\right) B_{i+1, \Xi}^{(p-1)}(t)\right]\right. \\
& =\sum_{i=2}^{n}\left[\omega_{i, p}(t) \psi_{i, p}(\tau)+\left(1-\omega_{i, p}(t)\right) \psi_{i-1, p}(\tau)\right] B_{i, \Xi}^{(p-1)}(t) \\
& =(t-\tau) \sum_{i=2}^{n} \psi_{i, p-1}(\tau) B_{i, \Xi}^{(p-1)}(t) \\
& =\ldots=(t-\tau)^{p} \sum_{i=p+1}^{n} \psi_{i, 0}(\tau) B_{i, \Xi}^{(0)}(t) .
\end{aligned}
$$

The assertion follows for $t \in\left[\xi_{p+1}, \xi_{n+1}\right)$ after dividing by $p$ ! and after deriving $\mu$ times with respect to $\tau$. Then, by continuity and by the assumption (33), the result holds for $t \in\left[\xi_{p+1}, \xi_{n+1}\right]$.

Lemma 2 immediately leads to the following properties.

- Marsden's identity. Using the Taylor expansion and formula (36), we obtain the representation of any polynomial of degree $p$ in terms of B-splines of the same degree, namely

$$
q(t)=\sum_{i=1}^{n} \Lambda_{i, p}(q) B_{i, \Xi}^{(p)}(t), \quad \forall q \in \mathbb{P}_{p}, \quad t \in\left[\xi_{p+1}, \xi_{n+1}\right],
$$

where

$$
\Lambda_{i, p}(q):=\sum_{r=0}^{p} \frac{(-1)^{r}}{p !} \frac{\mathrm{d}^{r} \psi_{i, p}(\tau)}{\mathrm{d} \tau^{r}} \frac{\mathrm{d}^{p-r} q(\tau)}{\mathrm{d} t^{p-r}} .
$$

- (Local) partition of unity. Considering $q(t)=1$, formula (39) provides

$$
1=\sum_{i=1}^{n} B_{i, \Xi}^{(p)}(t), \quad t \in\left[\xi_{p+1}, \xi_{n+1}\right] .
$$

Moreover, from (32) we get

$$
1=\sum_{i=r-p}^{r} B_{i, \Xi}^{(p)}(t), \quad t \in\left[\xi_{r}, \xi_{r+1}\right) .
$$

The local partition of unity is illustrated in Fig. 12 (right).

- Greville abscissas. Considering $q(t)=t$, formula (39) can be written as

$$
\Lambda_{i, p}(q)=\frac{\xi_{i+1}+\ldots+\xi_{i+p}}{p}=: \xi_{i, p}^{*},
$$


so that

$$
t=\sum_{i=1}^{n} \xi_{i, p}^{*} B_{i, \Xi}^{(p)}(t), \quad t \in\left[\xi_{p+1}, \xi_{n+1}\right] .
$$

The scalars $\xi_{i, p}^{*}$ defined in (40) are called again Greville abscissas ${ }^{11} 12$.

\subsubsection{Knot Multiplicity and Truncated Powers in $\mathbb{S}_{p, \Xi}$}

We now connect the multiplicity of the knots in $\Xi$ with the truncated powers which belong to the spline space $\mathbb{S}_{p, \Xi}$. The following lemma is similar to Lemma 2 .

Lemma 3. Let $\rho_{k}$ be the multiplicity of the knot $\xi_{k}$ as in (26), we have

$$
\frac{\left(t-\xi_{k}\right)_{+}^{p-\mu}}{(p-\mu) !}=\sum_{i=k}^{n} \frac{(-1)^{\mu}}{p !} \frac{\mathrm{d}^{\mu} \psi_{i, p}\left(\xi_{k}\right)}{\mathrm{d} \tau^{\mu}} B_{i, \Xi}^{(p)}(t), \quad t \in\left[\xi_{p+1}, \xi_{n+1}\right], \quad \forall 0 \leq \mu<\rho_{k},
$$

where we take the $\mu$-th order derivative of $\psi_{i, p}(\tau)$, defined in (37), and evaluate it at the knot $\xi_{k}$.

Proof. Let $i<k<i+p+1$, then from (37) we have that $\psi_{i, p}$ is a polynomial of degree $p$ with respect to $\tau$ with a zero of multiplicity $\rho_{k}$ at $\tau=\xi_{k}$. Hence,

$$
\frac{\mathrm{d}^{\mu} \psi_{i, p}\left(\xi_{k}\right)}{\mathrm{d} \tau^{\mu}}=0, \quad \mu=0, \ldots, \rho_{k}-1 .
$$

We obtain from (36) that for $t \in\left[\xi_{p+1}, \xi_{n+1}\right]$ and $\mu=0, \ldots, \rho_{k}-1$,

$$
\frac{\left(t-\xi_{k}\right)^{p-\mu}}{(p-\mu) !}=\sum_{i=k}^{n} \frac{(-1)^{\mu}}{p !} \frac{\mathrm{d}^{\mu} \psi_{i, p}\left(\xi_{k}\right)}{\mathrm{d} \tau^{\mu}} B_{i, \Xi}^{(p)}(t)+\sum_{i=1}^{k-p-1} \frac{(-1)^{\mu}}{p !} \frac{\mathrm{d}^{\mu} \psi_{i, p}\left(\xi_{k}\right)}{\mathrm{d} \tau^{\mu}} B_{i, \Xi}^{(p)}(t) .
$$

Therefore, from the compact support (31) and the definition of the truncated power function, we obtain

$$
\frac{\left(t-\xi_{k}\right)_{+}^{p-\mu}}{(p-\mu) !}=\sum_{i=k}^{n} \frac{(-1)^{\mu}}{p !} \frac{\mathrm{d}^{\mu} \psi_{i, p}\left(\xi_{k}\right)}{\mathrm{d} \tau^{\mu}} B_{i, \Xi}^{(p)}(t), \quad \mu=0, \ldots, \rho_{k}-1
$$

${ }^{11}$ An explicit expression of (40) was given by Thomas N.E. Greville in [33]. According to Isaac J. Schoenberg [57], Thomas N.E. Greville reviewed the paper [57] introducing some elegant simplifications.

${ }^{12}$ As in the case of Bernstein polynomials (Footnote 1), the Greville abscissas for B-splines can be used to define an interesting approximating operator, namely the so-called Schoenberg operator, see [57, Section 10]:

$$
Q(f, t):=\sum_{i=1}^{n} f\left(\xi_{i, p}^{*}\right) B_{i, \Xi}^{(p)}(t) .
$$

Analogously to the Bernstein operator, the Schoenberg operator reproduces polynomials of first degree and it is shape preserving, see Theorems 9 and 10. 
From Lemmas 2 and 3, it follows that polynomials in $\mathbb{P}_{p}$ and truncated powers of the form

$$
\left(.-\xi_{k}\right)_{+}^{v_{k}}, \quad p-\rho_{k}<v_{k} \leq p
$$

belong to $\mathbb{S}_{p, \Xi}$.

\subsubsection{B-Splines as a Basis for $\mathbb{P}_{p, X}^{\mathfrak{V}}$}

Combining the results of Lemmas 2 and 3, and taking into account (35), we know that the spline space $\mathbb{S}_{p, \Xi}$ includes the space $\mathbb{P}_{p, X}^{\mathfrak{V}}$, provided that each break point in $X$ appears in the knot sequence $\Xi$ with a suitable multiplicity. More precisely, a simple dimensional argument gives the following result.

Theorem 7 (Curry-Schoenberg, 1966). Assume the space $\mathbb{P}_{p, X}^{\mathfrak{V}}$ is given, and set $n:=\operatorname{dim}\left(\mathbb{P}_{p, X}^{\mathfrak{V}}\right)$, see (34). Let $\Xi:=\left\{\xi_{1} \leq \xi_{2} \leq \ldots \leq \xi_{n+p+1}\right\}$, so that

- $\xi_{1} \leq \ldots \leq \xi_{p+1}=x_{0}<\ldots<x_{l+1}=\xi_{n+1} \leq \ldots \leq \xi_{n+p+1}$,

- $x_{j}$ occurs $p+1-v_{j}$ times in $\Xi, j=1, \ldots, l$,

then $\left\{B_{1, \Xi}^{(p)}, \ldots, B_{n, \Xi}^{(p)}\right\}$ is a basis of $\mathbb{P}_{p, X}^{\mathfrak{V}}$ on $\left[\xi_{p+1}, \xi_{n+1}\right]$.

From the previous theorem we can derive the following properties.

- Smoothness at the knots. At a given knot $\xi_{k}$ of multiplicity $1 \leq \rho_{k} \leq p+1$, any element of $\mathbb{S}_{p, \Xi}$ is (at least) of class $C^{v_{k}-1}$ where

$$
p+1=v_{k}+\rho_{k}
$$

This property follows from the fact that $\left(t-\xi_{k}\right)_{+}^{\mu}$ is of class $C^{\mu-1}$ and from (35). We remark that (42) provides a simple rule to construct splines of a given degree and a certain smoothness at a given point. If smoothness not higher than $C^{\mu_{k}}$ is required at the knot $\xi_{k}$, then this knot must be of multiplicity $p-\mu_{k}$, see Fig. 14 .

- Minimal support. Since any element of $\mathbb{S}_{p, \Xi}$ is a piecewise polynomial of degree $p$, the end points of its support must coincide with two knots in $\Xi$. From the smoothness properties it can be proved that if the support of $f \in \mathbb{S}_{p, \Xi}$ is strictly contained in $\left[t_{i}, t_{i+p+1}\right]$ then $f \equiv 0$. Therefore, the B-splines have minimal support, see (31).

- (Local) linear independence. Since the set of B-splines is a basis of a space of dimension $n$, they are linearly independent. Moreover, they are locally linearly independent. In $\left[\xi_{r}, \xi_{r+1}\right)$ we have

$$
\left.\left\langle B_{r-p, \Xi}^{(p)}, \ldots, B_{r, \Xi}^{(p)}\right\rangle \equiv \mathbb{P}_{p}\right|_{\left[\xi_{r}, \xi_{r+1}\right)} .
$$

Hence, all nonvanishing B-splines on $\left[\xi_{r}, \xi_{r+1}\right)$ are linearly independent. It follows that any element of $\left.\mathbb{S}_{p, \Xi}\right|_{\left[\xi_{r}, \xi_{r+1}\right)}$ can be represented as a linear combination of Bernstein polynomials of degree $p$ on $\left[\xi_{r}, \xi_{r+1}\right)$. 

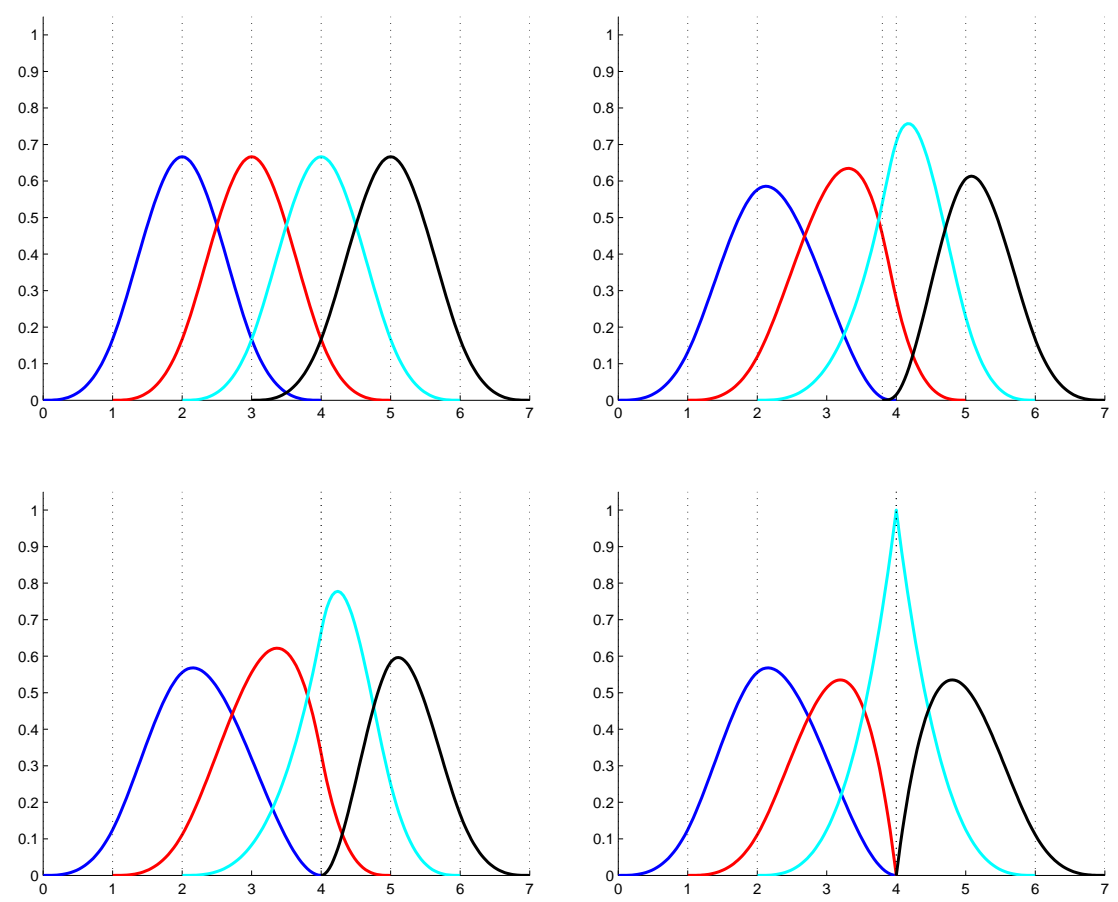

Fig. 14 Smoothness and knot multiplicity for cubic B-splines with different knot sequences $\Xi=$ $[0,1,2,3,4,5,6,7],[0,1,2,3.9,4,5,6,7],[0,1,2,4,4,5,6,7],[0,1,2,4,4,4,6,7]$.

- de Boor-Fix formula. The elements of the space $\mathbb{S}_{p, \Xi}$ can be represented as

$$
f(t)=\sum_{i=1}^{n} \Lambda_{i, p}(f) B_{i, \Xi}^{(p)}(t), \quad \forall f \in \mathbb{S}_{p, \Xi}
$$

where $^{13}$

$$
\Lambda_{i, p}(f):=\sum_{r=0}^{p} \frac{(-1)^{r}}{p !} \frac{\mathrm{d}^{r} \psi_{i, p}\left(\tau_{i}\right)}{\mathrm{d} \tau^{r}} \frac{\mathrm{d}^{p-r} f\left(\tau_{i}\right)}{\mathrm{d} t^{p-r}}, \quad \xi_{i}<\tau_{i}<\xi_{i+p+1}
$$

\subsection{Further Properties}

In this subsection we collect some further useful properties of B-splines.

${ }^{13}$ If $\tau_{i}$ in (44) is a knot, then the left or right derivatives of $f$ have to be considered. Actually, a slightly more general form of (44) can be given, see [8, Chapter IX]. 

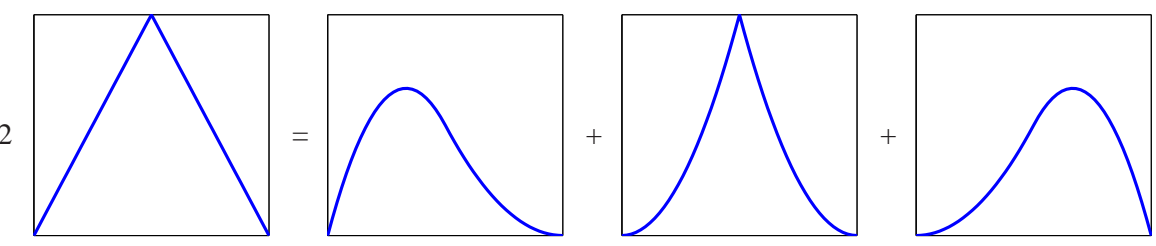

Fig. 15 Degree elevation for a linear B-spline.

- Derivative formulas. From Theorem 7 it follows that the derivative of one element in $\mathbb{S}_{p, \Xi}$ belongs to $\mathbb{S}_{p-1, \Xi}$. Therefore, by using the representation (43) we get

$$
\frac{\mathrm{d}}{\mathrm{d} t} f(t)=\sum_{i=2}^{n} \Lambda_{i, p-1}\left(\frac{\mathrm{d}}{\mathrm{d} t} f\right) B_{i, \Xi}^{(p-1)}(t), \quad \forall f \in \mathbb{S}_{p, \Xi}, \quad t \in\left[\xi_{p+1}, \xi_{n+1}\right] .
$$

By applying this to the basis elements and by taking into account that

$$
\psi_{i, p-1}(\tau)=\frac{\psi_{i, p}(\tau)-\psi_{i-1, p}(\tau)}{\xi_{i+p}-\xi_{i}}
$$

we obtain

$$
\frac{\mathrm{d}}{\mathrm{d} t} B_{i, \Xi}^{(p)}(t)=p\left[\frac{1}{\xi_{i+p}-\xi_{i}} B_{i, \Xi}^{(p-1)}(t)-\frac{1}{\xi_{i+p+1}-\xi_{i+1}} B_{i+1, \Xi}^{(p-1)}(t)\right]
$$

and

$$
\frac{\mathrm{d}}{\mathrm{d} t} \sum_{i=1}^{n} c_{i} B_{i, \Xi}^{(p)}(t)=\sum_{i=2}^{n} p \frac{c_{i}-c_{i-1}}{\xi_{i+p}-\xi_{i}} B_{i, \Xi}^{(p-1)}(t), \quad t \in\left[\xi_{p+1}, \xi_{n+1}\right] .
$$

Using the knot sequence proposed in Example 6, the above formulas can be compared $^{14}$ with (6) and (17).

- Integral recurrence relation. The derivative formula (45) immediately gives the following relation:

$$
B_{i, \Xi}^{(p)}(t)=d_{i, \Xi}^{(p-1)} \int_{-\infty}^{t} B_{i, \Xi}^{(p-1)}(s) \mathrm{d} s-d_{i+1, \Xi}^{(p-1)} \int_{-\infty}^{t} B_{i+1, \Xi}^{(p-1)}(s) \mathrm{d} s,
$$

where (see [59])

$$
d_{i, \Xi}^{(p)}:=\frac{1}{\int_{-\infty}^{+\infty} B_{i, \Xi}^{(p)}(s) \mathrm{d} s}=\frac{p+1}{\xi_{i+p+1}-\xi_{i}} .
$$

\footnotetext{
${ }^{14}$ To recover the polynomials $B_{i}^{(p-1)}$, only a multiplicity of $p$ is required, while for the polynomials
} $B_{i}^{(p)}$ a multiplicity of $p+1$ is needed. Therefore, there is a shift in indices comparing (6) and (45). 
Using the knot sequence proposed in Example 6, the recurrence relation (47) reduces to (11). Note that there is again a shift in indices comparing (11) and (47), see Footnote 14.

- Degree elevation. By using $\mathbb{P}_{p} \subset \mathbb{P}_{p+1}$ and by taking into account (42), we may consider the sequences of knots

$$
\Xi:=\left\{\ldots<\xi_{r}=\ldots=\xi_{r+\rho_{r}-1}<\ldots\right\} \subset\left\{\ldots<\xi_{r}=\ldots=\xi_{r+\rho_{r}}<\ldots\right\}=: \tilde{\Xi} .
$$

Therefore, $\mathbb{S}_{p, \Xi} \subset \mathbb{S}_{p+1, \tilde{\Xi}}$. In particular, it can be proved (see [54, Sect. 6.5])

$$
B_{i,\left[\xi_{i}, \ldots, \xi_{i+p+1}\right]}^{(p)}=\frac{1}{p+1} \sum_{r=i}^{i+p+1} B_{r,\left[\xi_{i}, \ldots, \xi_{r}, \xi_{r}, \ldots, \xi_{i+p+1}\right]}^{(p+1)} .
$$

This degree elevation is illustrated in Fig. 15.

\subsubsection{Interpolation in $\mathbb{S}_{p, \Xi}$}

Interpolation is probably the most popular way to obtain an element in a given space of functions. Here, we are interested in constructing interpolants belonging to $\mathbb{S}_{p, \Xi}$. More precisely, we want to solve the following problem.

Problem 1. Let $\left(t_{1}, f_{1}\right),\left(t_{2}, f_{2}\right), \ldots,\left(t_{n}, f_{n}\right)$ be given. Find $f \in \mathbb{S}_{p, \Xi}$ such that

$$
f\left(t_{i}\right)=f_{i}, \quad i=1, \ldots, n .
$$

It is well-known that interpolation in the polynomial case has a unique solution if and only if the interpolation points are different. However, this requirement is not enough to get unisolvency for Problem 1. One can easily construct a counterexample. For instance, since $\left.f\right|_{\left[\xi_{r}, \xi_{r+1}\right)} \in \mathbb{P}_{p}$, Problem 1 cannot be unisolvent if $n>p+1$ and all the interpolation points $t_{1}<t_{2}<\ldots<t_{n}$ belong to the knot interval $\left[\xi_{r}, \xi_{r+1}\right)$.

The following elegant result characterizes the distribution of the interpolation points such that Problem 1 has a unique solution, see [8, Chapter XIII].

Theorem 8 (Schoenberg-Whitney, 1953). Let $t_{1}<t_{2}<\ldots<t_{n}$ be given, such that if $t_{i}$ is a knot it has multiplicity at most $p$. Then, Problem 1 has a unique solution if and only if

$$
B_{i, \Xi}^{(p)}\left(t_{i}\right)>0, \quad i=1, \ldots, n .
$$

From the previous theorem we obtain that the interpolation problem is unisolvent if the interpolation points are "well distributed". Actually, each basis function needs at least one interpolation point in its support.

Example 7. If the interpolation points are taken as the Greville abscissas, then Problem 1 is unisolvent.

Remark 3. From Theorem 8 it follows that the B-spline collocation matrix (see (21)) at the points $t_{1}, \ldots, t_{n}$ is nonsingular if and only if it has a strictly positive diagonal. 


\subsection{Geometrical and Graphical Properties}

The parametric curve

$$
\mathcal{C}(t):=\sum_{i=1}^{n} \mathbf{C}_{i} B_{i, \Xi}^{(p)}(t), \quad \mathbf{C}_{i} \in \mathbb{R}^{d}
$$

is referred to as a $B$-spline curve, and the points $\mathbf{C}_{i}$ are its control points. The graph of any spline function

$$
f(t):=\sum_{i=1}^{n} c_{i} B_{i, \Xi}^{(p)}(t)
$$

in $\mathbb{S}_{p, \Xi}$ can be seen as a planar curve

$$
\left(\begin{array}{c}
t \\
f(t)
\end{array}\right)=\sum_{i=1}^{n}\left(\begin{array}{c}
\xi_{i, p}^{*} \\
c_{i}
\end{array}\right) B_{i, \Xi}^{(p)}(t), \quad t \in\left[\xi_{p+1}, \xi_{n+1}\right],
$$

where $\xi_{i, p}^{*}$ are the Greville abscissas (40). The polygonal line connecting the points $\mathbf{C}_{i} \in \mathbb{R}^{d}$ (or the points $\left(\begin{array}{c}\xi_{i, p}^{*} \\ c_{i}\end{array}\right)$ in the plane) is the control polygon of $\mathcal{C}$ (or $f$ ).

\subsubsection{Main Properties of B-Spline Curves}

The following properties follow easily from the properties of B-splines mentioned before.

- (Local) convex hull. Given (50) with $t \in\left[\xi_{r}, \xi_{r+1}\right)$, the positivity and (local) partition of unity of B-splines imply that only $\mathbf{C}_{r-p}, \ldots, \mathbf{C}_{r-1}, \mathbf{C}_{r}$ act on $\mathcal{C}(t)$, and that

$$
\mathcal{C}(t) \in \mathcal{H}\left(\mathbf{C}_{r-p}, \ldots, \mathbf{C}_{r-1}, \mathbf{C}_{r}\right),
$$

see Fig. 16 (left). In case of scalar functions (52) with $t \in\left[\xi_{r}, \xi_{r+1}\right)$, only $c_{r-p}, \ldots, c_{r-1}, c_{r}$ have an effect on $f(t)$, see Fig. 16 (right).

- Tangent end conditions. Let us consider the knot sequence (27) and (51). The partition of unity, the smoothness relation (42), the Greville abscissas (40) and the derivative formula (46) lead to

$$
\begin{aligned}
& f\left(\xi_{p+1}\right)=c_{1}, \quad \frac{\mathrm{d}}{\mathrm{d} t} f\left(\xi_{p+1}\right)=\frac{c_{2}-c_{1}}{\xi_{2, p}^{*}-\xi_{1, p}^{*}}, \\
& f\left(\xi_{n+1}\right)=c_{n}, \quad \frac{\mathrm{d}}{\mathrm{d} t} f\left(\xi_{n+1}\right)=\frac{c_{n}-c_{n-1}}{\xi_{n, p}^{*}-\xi_{n-1, p}^{*}} .
\end{aligned}
$$

Hence, the control polygon is tangent to $f$ at the end points $\xi_{p+1}$ and $\xi_{n+1}$, see Fig. 16 (right). 

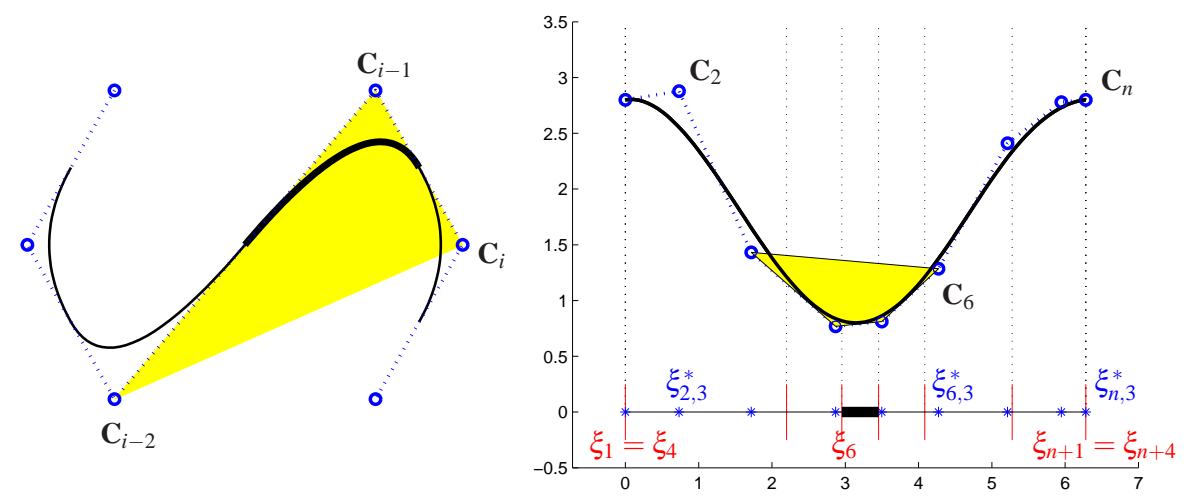

Fig. 16 Local convex hull for a quadratic curve (left) and a cubic spline function (right).

- de Boor algorithm. The B-spline curve (50) with $t \in\left[\xi_{r}, \xi_{r+1}\right)$ can be written by using (30) and (32) as

$$
\begin{aligned}
\sum_{i=1}^{n} \mathbf{C}_{i}^{[p]} B_{i, \Xi}^{(p)}(t) & =\sum_{i=r-p}^{r} \mathbf{C}_{i}^{[p]} B_{i, \Xi}^{(p)}(t) \\
& =\sum_{i=r-p}^{r} \mathbf{C}_{i}^{[p]}\left[\omega_{i, p}(t) B_{i, \Xi}^{(p-1)}(t)+\left(1-\omega_{i+1, p}(t)\right) B_{i+1, \Xi}^{(p-1)}(t)\right] \\
& =\sum_{i=r-p+1}^{r} \mathbf{C}_{i}^{[p-1]} B_{i, \Xi}^{(p-1)}(t)=\ldots=\sum_{i=r}^{r} \mathbf{C}_{i}^{[0]} B_{i, \Xi}^{(0)}(t)=\mathbf{C}_{r}^{[0]}
\end{aligned}
$$

where at each step the new coefficients are obtained by a convex combination,

$$
\mathbf{C}_{i}^{[k-1]}:=\omega_{i, k}(t) \mathbf{C}_{i}^{[k]}+\left(1-\omega_{i, k}(t)\right) \mathbf{C}_{i-1}^{[k]}, \quad k=p, p-1, \ldots, 1
$$

This evaluation procedure is known as the de Boor algorithm ${ }^{15}$.

Using the knot sequence proposed in Example 6, the relation (53) reduces to (15). Note that there is a shift in indices comparing (15) and (53), see Footnote 14.

\subsubsection{Knot Insertion}

Let us consider the insertion of a new knot $\bar{\xi}$ in the knot sequence $\Xi$, namely

$$
\Xi:=\left\{\ldots \leq \xi_{i} \leq \xi_{i+1} \leq \ldots\right\} \subset\left\{\ldots \leq \xi_{i} \leq \bar{\xi} \leq \xi_{i+1} \leq \ldots\right\}=: \bar{\Xi},
$$

${ }^{15}$ As in the case of Bézier curves, the development of a stable evaluation algorithm for B-spline curves is related to the car industry. In the early 1960s, Carl de Boor worked at General Motors Research, where splines were already being employed in automotive design. There he laid the foundations of his famous algorithm, see [6]. 

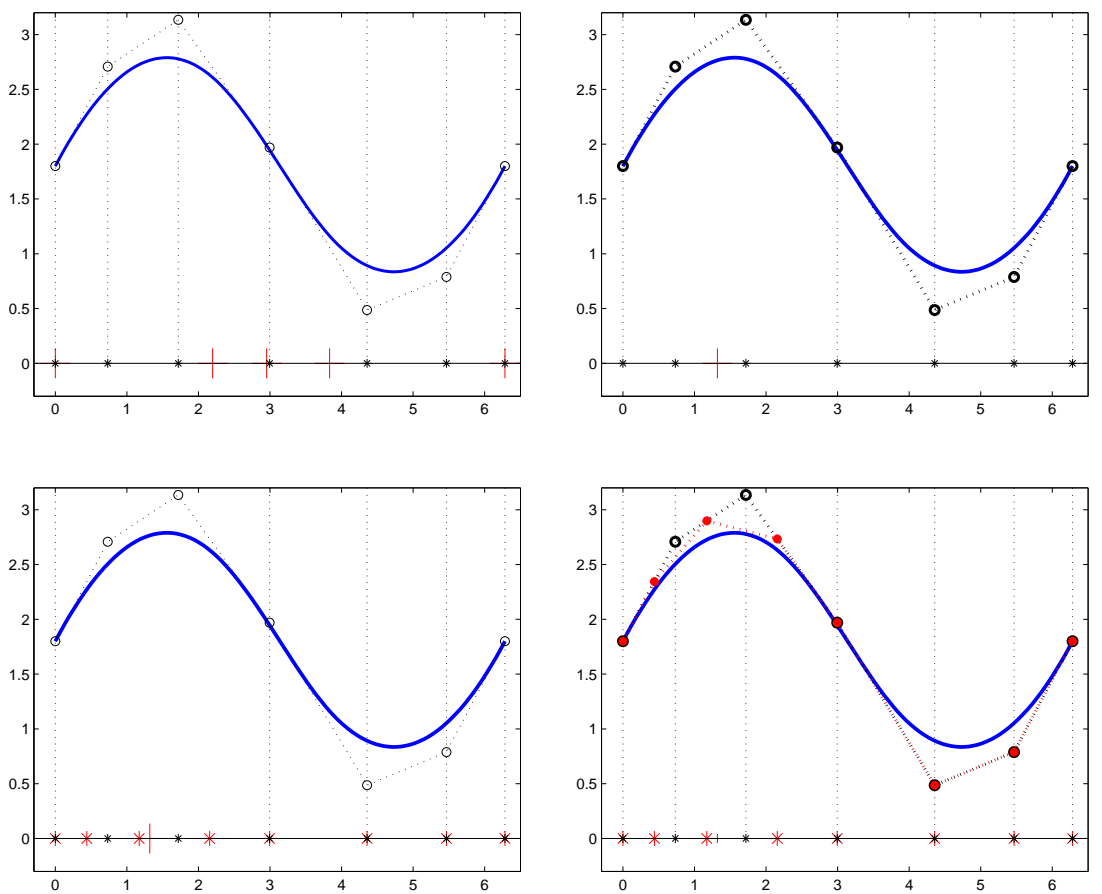

Fig. 17 Knot insertion for cubic B-splines with a knot sequence of the form (27). Top left: the curve with the original control polygon. Top right: a new knot between 1 and 2 is inserted. Bottom left: the new Greville abscissas are indicated by red stars $(*)$. Bottom right: the new control polygon is obtained by interpolating the old one at the new Greville abscissas.

then $\mathbb{S}_{p, \Xi} \subset \mathbb{S}_{p, \bar{\Xi}}$, so that

$$
\sum_{j=1}^{n} \mathbf{C}_{j} B_{j, \Xi}^{(p)}=\sum_{j=1}^{n+1} \overline{\mathbf{C}}_{j} B_{j, \bar{\Xi}}^{(p)}
$$

Applying (43) to both sides of (55), we obtain

$$
\overline{\mathbf{C}}_{j}=\bar{\omega}_{j, p} \mathbf{C}_{j}+\left(1-\bar{\omega}_{j, p}\right) \mathbf{C}_{j-1}, \quad \bar{\omega}_{j, p}:= \begin{cases}1, & \xi_{j+p} \leq \bar{\xi} \\ \frac{\bar{\xi}-\xi_{j}}{\xi_{j+p}-\xi_{j}}, & \xi_{j}<\bar{\xi}<\xi_{j+p}, \\ 0, & \bar{\xi} \leq \xi_{j} .\end{cases}
$$

We note that (56) has a nice geometric interpretation. In the scalar case the first components of the control points are the Greville abscissas. Therefore, (56) provides the Greville abscissas for the new sequence of knots $\bar{\Xi}$. It follows that the new control polygon interpolates the old one at the new Greville abscissas, see Fig. 17. Since any 

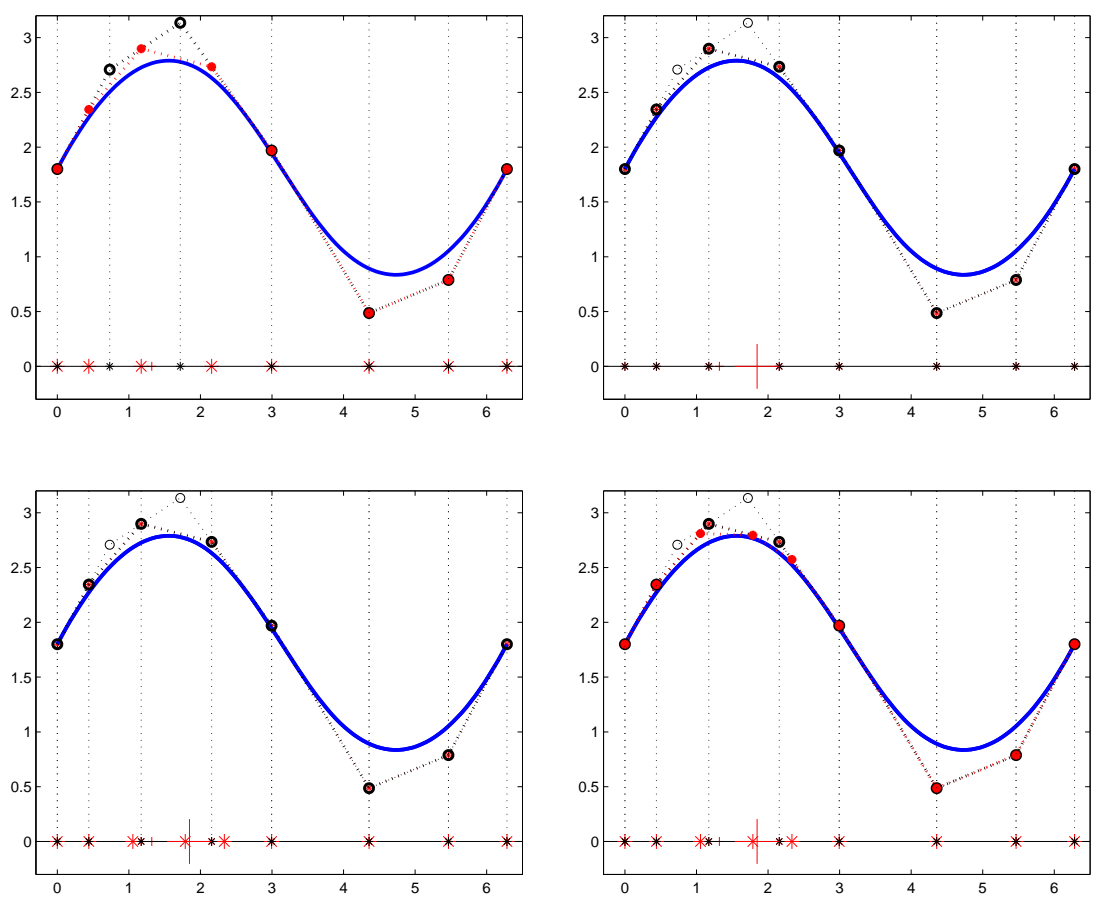

Fig. 18 Iterated knot insertion for cubic B-splines.

component of (50) belongs to $\mathbb{S}_{p, \Xi}$, it can be represented as (52). Hence, the above geometric interpretation applies component-wisely for the general curve case.

As a consequence, the new control polygon is obtained by corner cutting from the old one, see see Fig. 17 (bottom right).

Remark 4. Note that (56) is nothing else than applying one step of the de Boor algorithm, see (53). It follows that the de Boor algorithm can be seen as repeated corner cuttings.

Remark 5. The (single) knot insertion can be iterated, see Fig. 18. The finer the knot sequence, the closer is the control polygon to the function being represented. There is convergence under knot refinement. The convergence order is $\mathcal{O}\left(h^{2}\right)$ with $h$ the maximum knot distance, see [8, Chapter XI]. Knot insertion in CAGD corresponds to $h$-refinement in IgA.

Remark 6. When several knots have to be inserted simultaneously, more efficient algorithms can be used instead of repeating (56), e.g., the Oslo algorithm [16].

Remark 7. A fast and elegant way to visualize spline curves is based on repeating knot insertion: after inserting a few times a knot between each pair of existing knots, 
it is sufficient to draw the control polygon. In the case of equally spaced knots, this procedure basically leads to classical subdivision schemes, see [23].

Remark 8. Let us consider the repeated insertion of a point $x$ as a knot, $p$ times, so that for some $r$,

$$
\xi_{r}<\xi_{r+1}=\ldots=\xi_{r+p}=x<\xi_{r+p+1} .
$$

Then, from (31) and the partition of unity property, we obtain

$$
B_{j, \Xi}^{(p)}(x)= \begin{cases}1, & j=r \\ 0, & \text { otherwise }\end{cases}
$$

so that

$$
\sum_{j=1}^{n} c_{j} B_{j, \Xi}^{(p)}(x)=c_{r} B_{r, \Xi}^{(p)}(x)=c_{r} .
$$

Remark 9. Let us consider a knot interval $\left[\xi_{r}, \xi_{r+1}\right]$, and a (possibly) repeated insertion in the knot sequence of $\xi_{r}$ and $\xi_{r+1}$ so that each of them has multiplicity ${ }^{16}$ $p+1$. Then, the elements of the corresponding B-spline sequence not vanishing on $\left[\xi_{r}, \xi_{r+1}\right]$ are the Bernstein polynomials (see Example 6). Hence, the (repeated) knot insertion provides the conversion from the B-spline form to the Bézier-Bernstein form. This conversion is called Bézier extraction.

Remark 10. The degree elevation (49) also requires a knot insertion procedure, see (48). Repeated degree elevation leads to a sequence of control polygons that converges to the B-spline curve with a convergence order of $\mathcal{O}(1 / p)$, see [54, Sect. 6.6]. Degree elevation in CAGD corresponds to $p$-refinement in IgA.

\subsubsection{Variation Diminishing and Total Positivity}

Let us consider two sequences of knots $\Xi \subset \check{\Xi}$, and an element (51) in $\mathbb{S}_{p, \Xi}$, then $f$ belongs to $\mathbb{S}_{p, \check{\Xi}}$ with the following representation

$$
f(t)=\sum_{i=1}^{\check{n}} \check{c}_{i} B_{i, \check{\Xi}}^{(p)}(t)
$$

Since the new control polygon of $f$ is obtained by repeated corner cuttings from the old one, see (56), it follows that the number of sign changes in $\left\{\check{c}_{1}, \ldots, \check{c}_{\breve{n}}\right\}$ is bounded above by the number of sign changes in $\left\{c_{1}, \ldots, c_{n}\right\}$, i.e.,

$$
S^{-}\left(\check{c}_{1}, \ldots, \check{c}_{\check{n}}\right) \leq S^{-}\left(c_{1}, \ldots, c_{n}\right),
$$

see Sect. 2.2.1. Moreover, we have the following result.

Theorem 9 (Schoenberg, 1967). The number of sign changes of $f$ defined in (51) is bounded above by the number of sign changes in the sequence of its coefficients.

${ }_{16}$ A multiplicity $p$ is actually sufficient for Bézier extraction. 
Proof. Let $t_{1}<\ldots<t_{r}$ be any sequence of evaluation points. Then, we insert each $t_{i}, i=1, \ldots, r$ in the knot sequence $\Xi$, so that $t_{i}$ is a knot of multiplicity $p$. Let us denote by $\Xi$ the new sequence of knots. From Remark 8 we have that

$$
f\left(t_{1}\right), \ldots, f\left(t_{r}\right)
$$

is a subsequence of the sequence of the coefficients $\left\{\check{c}_{1}, \ldots, \check{c}_{\check{n}}\right\}$ representing $f$ in $\mathbb{S}_{p, \check{\Xi}}$. Therefore, from (57) we obtain

$$
S^{-}\left(f\left(t_{1}\right), \ldots, f\left(t_{r}\right)\right) \leq S^{-}\left(\check{c}_{1}, \ldots, \check{c}_{\check{n}}\right) \leq S^{-}\left(c_{1}, \ldots, c_{n}\right)
$$

The previous theorem ensures that the B-spline basis provides a variation diminishing representation (see Corollary 1 and Proposition 2). In particular, the number of zeros of an element in $\mathbb{S}_{p, \Xi}$ can be bounded above in terms of the number of zeros in its control polygon.

The following fundamental theorem can be proved by knot insertion, see [8, Chapter XIII].

Theorem 10 (Karlin). The B-spline basis is NTP.

Moreover, by using Theorem 6, we arrive at the following result, see [13].

Theorem 11. The B-spline basis is the ONTP basis for piecewise polynomials.

Summarizing, the above results in this section ensure that the B-spline basis is the best basis for the space of piecewise polynomials $\mathbb{P}_{p, X}^{\mathfrak{V}}$.

Remark 11. All the mentioned B-spline properties can be compactly obtained by means of the so-called blossoming principle [47, 56].

\subsection{Tensor-Product B-Splines}

The most easy way to extend many of the previous results to the multivariate environment is to consider a so-called tensor-product approach. For the sake of simplicity, we briefly focus here on the bivariate setting. The extension to higher dimensions is straightforward; it only requires a more involved notation, see [34] for further details.

Given two knot sequences

$$
\Xi:=\left\{\xi_{1} \leq \xi_{2} \leq \ldots \leq \xi_{n+p+1}\right\}, \quad \Upsilon:=\left\{v_{1} \leq v_{2} \leq \ldots \leq v_{m+q+1}\right\},
$$

tensor-product B-spline surfaces can be easily constructed as follows

$$
\mathcal{S}(t, s):=\sum_{i=1}^{n} \sum_{j=1}^{m} \mathbf{C}_{i, j} B_{i, \Xi}^{(p)}(t) B_{j, Y}^{(q)}(s), \quad \mathbf{C}_{i, j} \in \mathbb{R}^{d} .
$$



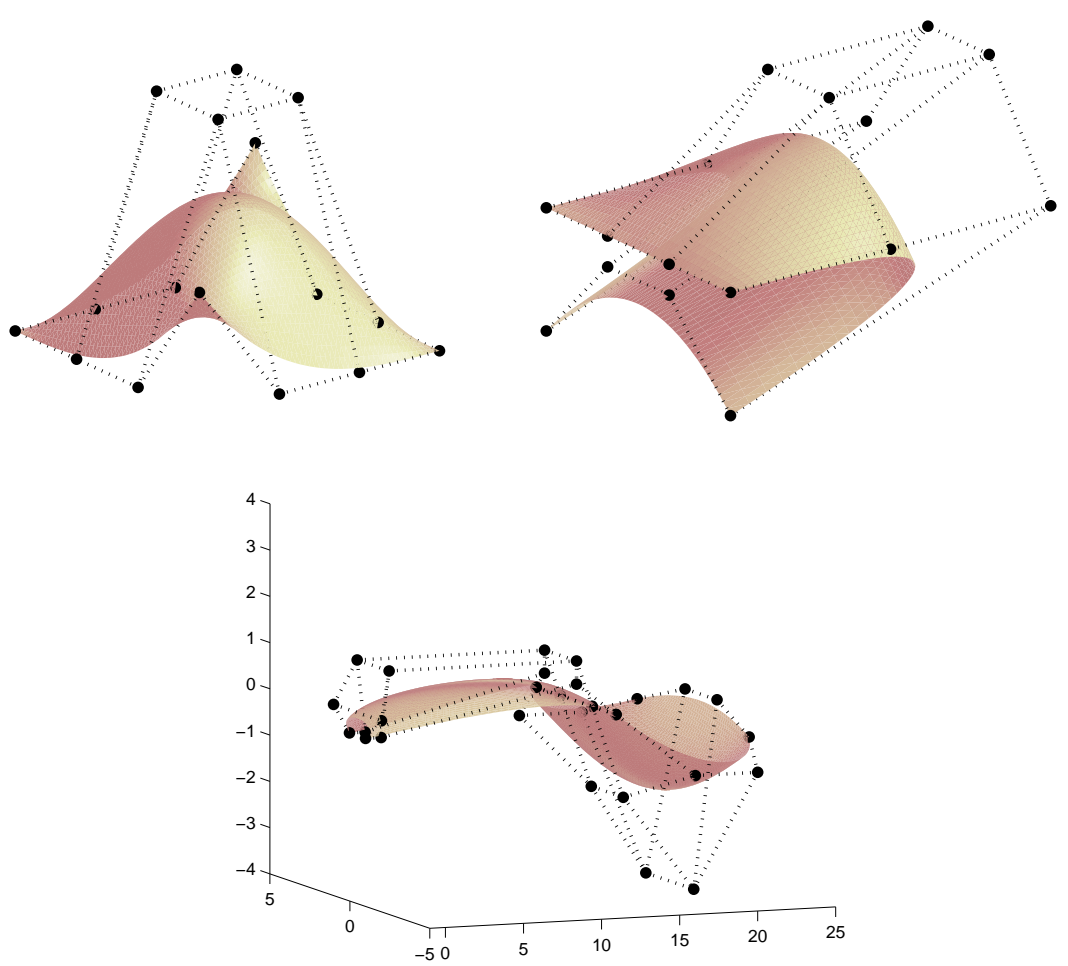

Fig. 19 Some tensor-product bicubic $C^{1}$ surfaces and the corresponding control nets.

The points $\mathbf{C}_{i, j}$ are the control points, and they can be organized in a rectangular control net, see Fig. 19. The graph of any tensor-product spline function

$$
f(t, s):=\sum_{i=1}^{n} \sum_{j=1}^{m} c_{i, j} B_{i, \Xi}^{(p)}(t) B_{j, \Upsilon}^{(q)}(s)
$$

can be seen as a surface in $\mathbb{R}^{3}$ for $(t, s) \in\left[\xi_{p+1}, \xi_{n+1}\right] \times\left[v_{q+1}, v_{m+1}\right]$, namely

$$
\left(\begin{array}{c}
t \\
s \\
f(t, s)
\end{array}\right)=\sum_{i=1}^{n} \sum_{j=1}^{m}\left(\begin{array}{c}
\xi_{i, p}^{*} \\
v_{j, q}^{*} \\
c_{i, j}
\end{array}\right) B_{i, \Xi}^{(p)}(t) B_{j, r}^{(q)}(s),
$$

where $\xi_{i, p}^{*}$ and $v_{j, q}^{*}$ are the Greville abscissas for univariate B-splines of degree $p$ and $q$, corresponding to the two knot sequences $\Xi$ and $\Upsilon$, respectively.

It is clear that the above tensor-product representation inherits all the properties (positivity, partition of unity, local convex hull, tangent end conditions) of the univariate case. In addition, a main advantage is that evaluation of (58) can be reduced 
to a sequence of evaluations of B-spline curves:

$$
\mathcal{S}(t, s)=\sum_{i=1}^{n} \mathbf{D}_{i, s} B_{i, \Xi}^{(p)}(t), \quad \mathbf{D}_{i, s}:=\sum_{j=1}^{m} \mathbf{C}_{i, j} B_{j, \Upsilon}^{(q)}(s),
$$

or equivalently

$$
\mathcal{S}(t, s)=\sum_{j=1}^{m} \mathbf{D}_{j, t} B_{j, Y}^{(q)}(s), \quad \mathbf{D}_{j, t}:=\sum_{i=1}^{n} \mathbf{C}_{i, j} B_{i, \Xi}^{(p)}(t) .
$$

Note that (60) requires $n$ B-spline curve evaluations of degree $q$ and one B-spline curve evaluation of degree $p$. On the other hand, (61) requires $m \mathrm{~B}$-spline curve evaluations of degree $p$ and one B-spline curve evaluation of degree $q$. Thus, it is better to choose one of the two forms according to the minimal computational cost.

Despite their simple and elegant formulation, tensor-product structures have a main drawback. Any refinement of a knot sequence has a global effect in the other direction, so they prevent to do local refinement. Later on, see Sect. 5, we describe how to define B-splines on suitable triangulations, which naturally support local mesh refinements.

\subsection{NURBS: Non-Uniform Rational B-Splines}

In this subsection we summarize the definition and main properties of the rational extension of B-splines, the so-called NURBS. NURBS are the de facto standard in commercial CAD (Computer Aided Design) systems, because they allow an exact representation of conic segments while maintaining several nice properties of the B-splines.

\subsubsection{Definition and Main Properties}

Given a set of B-splines $\left\{B_{i, \Xi}^{(p)}(t), i=1, \ldots, n\right\}$ and a corresponding set of strictly positive weights $^{17}$,

$$
W:=\left\{w_{i}>0, i=1, \ldots, n\right\}
$$

we state the following definition.

Definition 9. NURBS basis functions are given by

$$
R_{i, \Xi, W}^{(p)}(t):=\frac{w_{i} B_{i, \Xi}^{(p)}(t)}{\sum_{j=1}^{n} w_{j} B_{j, \Xi}^{(p)}(t)} .
$$

${ }^{17}$ Negative and zero weights can be used as well. Here, we just consider positive weights because they ensure nice properties of the resulting functions. 
Referring to Sect. 3.1 and (42), NURBS immediately inherit from B-splines the following properties.

- Positivity.

- Partition of unity.

- Compact support.

- Smoothness related to knot multiplicity.

In addition, we have the following result.

Theorem 12. The NURBS basis is NTP.

Proof. Assume the values $t_{1}<t_{2} \ldots<t_{r}$ are given, and set

$$
\mathcal{W}_{\Xi, W}^{(p)}(t):=\frac{1}{\sum_{j=1}^{n} w_{j} B_{j, \Xi}^{(p)}(t)} .
$$

The NURBS collocation matrix

$$
\left(\begin{array}{ccc}
R_{1, \Xi, W}^{(p)}\left(t_{1}\right) & \ldots & R_{n, \Xi, W}^{(p)}\left(t_{1}\right) \\
\vdots & & \vdots \\
R_{1, \Xi, W}^{(p)}\left(t_{r}\right) & \ldots & R_{n, \Xi, W}^{(p)}\left(t_{r}\right)
\end{array}\right)
$$

is the product of the following three matrices

$$
\left(\begin{array}{cccc}
\mathcal{W}_{\Xi, W}^{(p)}\left(t_{1}\right) & 0 & \ldots & 0 \\
0 & \mathcal{W}_{\Xi, W}^{(p)}\left(t_{2}\right) & \ldots & 0 \\
\vdots & \vdots & & \vdots \\
0 & 0 & \ldots & \mathcal{W}_{\Xi, W}^{(p)}\left(t_{r}\right)
\end{array}\right)\left(\begin{array}{ccc}
B_{1, \Xi}^{(p)}\left(t_{1}\right) & \ldots & B_{n, \Xi}^{(p)}\left(t_{1}\right) \\
\vdots & & \vdots \\
B_{1, \Xi}^{(p)}\left(t_{r}\right) & \ldots & B_{n, \Xi}^{(p)}\left(t_{r}\right)
\end{array}\right)\left(\begin{array}{cccc}
w_{1} & 0 & \ldots & 0 \\
0 & w_{2} & \ldots & 0 \\
\vdots & \vdots & & \vdots \\
0 & 0 & \ldots & w_{n}
\end{array}\right) .
$$

From Theorem 10 we know that the B-spline basis is TP. Thus, the matrix (62) is the product of TP matrices and so it is TP as well, see Theorem 2. Normalization comes from the partition of unity.

A NURBS curve is defined by

$$
\sum_{i=1}^{n} \mathbf{C}_{i} R_{i, \Xi, W}^{(p)}(t), \quad \mathbf{C}_{i} \in \mathbb{R}^{d}
$$

It can be seen as a projective transformation of a B-spline curve in $\mathbb{R}^{d+1}$,

$$
\left(\sum_{i=1}^{n} \mathbf{C}_{i} w_{i} B_{i, \Xi}^{(p)}(t), \sum_{i=1}^{n} w_{i} B_{i, \Xi}^{(p)}(t)\right) .
$$


Given two knot sequences

$$
\Xi:=\left\{\xi_{1} \leq \xi_{2} \leq \ldots \leq \xi_{n+p+1}\right\}, \quad \Upsilon:=\left\{v_{1} \leq v_{2} \leq \ldots \leq v_{m+q+1}\right\},
$$

tensor-product NURBS surfaces can be easily constructed as

$$
\mathcal{S}(t, s):=\sum_{i=1}^{n} \sum_{j=1}^{m} \mathbf{C}_{i, j} R_{i, j, \Xi, Y}^{(p, q)}(t, s), \quad \mathbf{C}_{i, j} \in \mathbb{R}^{d},
$$

with

$$
R_{i, j, \Xi, Y}^{(p, q)}(t, s):=\frac{w_{i, j} B_{i, \Xi}^{(p)}(t) B_{j, \Upsilon}^{(q)}(s)}{\sum_{k=1}^{n} \sum_{l=1}^{m} w_{k, l} B_{k, \Xi}^{(p)}(t) B_{l, \Upsilon}^{(q)}(s)}, \quad w_{i, j}>0 .
$$

For further details, we refer to [52].

\subsubsection{Representation of Arcs of Conic Sections}

NURBS are of interest because they allow an exact representation of segments of conic sections. Here we focus on this property, considering the simplest case, namely a single quadratic rational segment:

$$
\frac{(1-t)^{2} w_{1} \mathbf{C}_{1}+2 t(1-t) w_{2} \mathbf{C}_{2}+t^{2} w_{3} \mathbf{C}_{3}}{(1-t)^{2} w_{1}+2 t(1-t) w_{2}+t^{2} w_{3}} .
$$

We now show the relation between the weights $w_{1}, w_{2}, w_{3}$ in (64) and the conic section to be represented. In order to determine which kind of conic section we are dealing with, we compute the points at infinity of the conic in the projective plane. To this end, we look for the zeros of the third component in the homogeneous form (63), i.e. the denominator in (64). A straightforward computation gives that

$$
(1-t)^{2} w_{1}+2 t(1-t) w_{2}+t^{2} w_{3}=0
$$

has two distinct real roots, one double real root, or two complex conjugate roots, according to the sign of

$$
w_{2}^{2}-w_{1} w_{3}
$$

This immediately results in the following theorem.

Theorem 13. Setting

$$
\kappa:=\frac{w_{1} w_{3}}{w_{2}^{2}}
$$

we have the following characterization of the conic segment in (64):

$$
\kappa= \begin{cases}<1 & \text { hyperbola } \\ =1 & \text { parabola } \\ >1 & \text { ellipse }\end{cases}
$$


The scalar $\kappa$ in (65) is called the conic shape factor.

Remark 12. The same conic segment can be represented by using different weights in (64). Actually, two weights in (64) can be arbitrarily fixed. Indeed, it can be seen that whenever the weights are selected so that $\kappa$ maintains the same value, (64) gives a different parameterization of the same conic segment. Usually one takes $w_{1}=w_{3}=1$.

\section{Beyond Polynomials}

The most interesting properties of Bernstein polynomials and B-splines are not restricted to polynomials or piecewise polynomials, but they are related to more general properties of the considered spaces. In this section we deepen this aspect and we consider spaces which are the natural extension of polynomials, namely extended Chebyshev spaces ${ }^{18}$, see [59]. In particular, we discuss how optimal bases can be constructed for these spaces and how we can generalize the concept of B-splines beyond the polynomial case.

\subsection{Extended Chebyshev Spaces}

B-splines are a basis for piecewise polynomials, i.e. piecewise functions with segments belonging to the space $\mathbb{P}_{p}=\left\langle 1, t, \ldots, t^{p-2}, t^{p-1}, t^{p}\right\rangle$. It is natural to replace such a space with some more general ones.

Definition 10. Let $\mathbb{E}$ be a space of dimension $p+1$ defined on an interval $I . \mathbb{E}$ is an extended Chebyshev (EC) space if any non-trivial element has at most p zeros in I, including their multiplicity.

Classical examples are the null spaces of linear differential operators of order $p+1$ with constant coefficients. They are EC spaces on $I=\mathbb{R}$ when their characteristic polynomials have only real roots. If the characteristic polynomial has at least one non-real root, then the null space is an EC space only on a sufficiently small interval (at least on any interval of length $\frac{\pi}{a}$ where $a$ denotes the greatest imaginary part of all non-real roots of the characteristic polynomial).

Theorem 14 ([48]). Let $\mathbb{E}$ be any $p+1$ dimensional subspace of $C^{p}(I)$, containing constants, such that the Wronskian of any basis of $\mathbb{E}$ does not vanish on the given interval. ${ }^{19}$ Then the following properties are equivalent:

- $\mathbb{E}$ possesses an ONTP basis on any $(c, d) \subset I$;

- $\mathbb{E}^{\prime}:=\left\{f^{\prime}: f \in \mathbb{E}\right\}$ is a $p$ dimensional EC space on $I$.

${ }^{18}$ For a more general approach we refer to [32].

19 This is always the case for an EC space. 

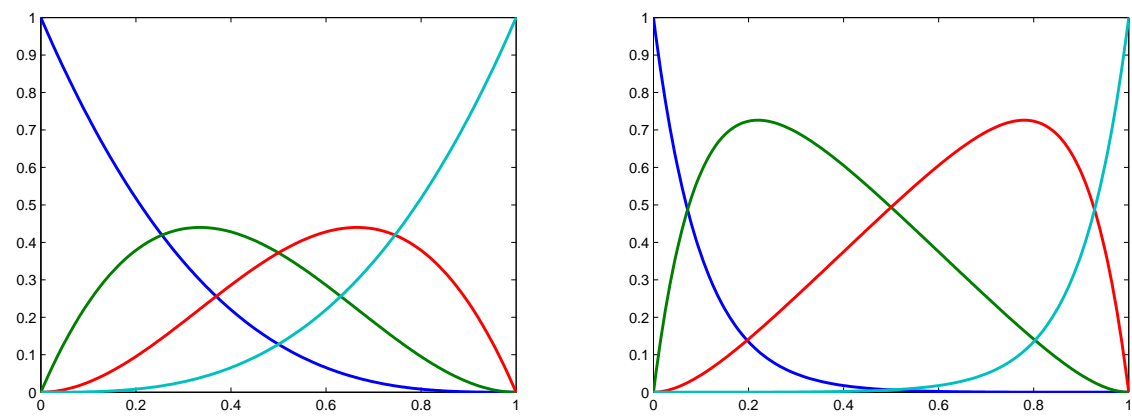

Fig. 20 The ONTP basis of $\mathbb{P}_{3}^{u, v}$. Left: $\langle u, v\rangle=\left\langle\cos \left(\frac{\pi}{4} t\right), \sin \left(\frac{\pi}{4} t\right)\right\rangle$. Right: $\langle u, v\rangle=$ $\langle\cosh (10 t), \sinh (10 t)\rangle$.
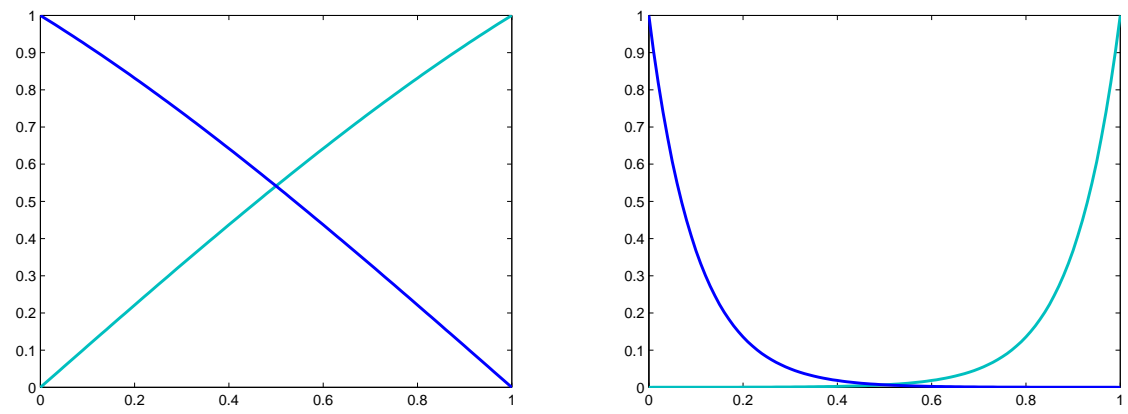

Fig. $21 B_{0, u, v}^{(1)}$ and $B_{1, u, v}^{(1)}$. Left: $\langle u, v\rangle=\left\langle\cos \left(\frac{\pi}{4} t\right), \sin \left(\frac{\pi}{4} t\right)\right\rangle$. Right: $\langle u, v\rangle=\langle\cosh (10 t), \sinh (10 t)\rangle$.

Spaces of the form $\mathbb{E}=\mathbb{P}_{p}^{u, v}$, where

$$
\mathbb{P}_{p}^{u, v}:=\left\langle 1, t, \ldots, t^{p-2}, u(t), v(t)\right\rangle, p \geq 2, t \in I,
$$

are of special interest because they combine a "polynomial structure" with the ability of exactly representing salient profiles.

Let us assume $u, v \in C^{p}$ and let us denote

$$
U:=\frac{\mathrm{d}^{p-1}}{\mathrm{~d} t^{p-1}} u, \quad V:=\frac{\mathrm{d}^{p-1}}{\mathrm{~d} t^{p-1}} v .
$$

In the following we assume

$$
\langle U, V\rangle \text { is an EC space on } I,
$$

so that $\mathbb{P}_{p}^{u, v}$ is an EC space on $I$. 
- The ONTP basis of $\mathbb{P}_{p}^{u, v}$. Thanks to Theorem 14 we know that the space $\mathbb{P}_{p}^{u, v}$ possesses an ONTP basis, denoted by

$$
\left\{B_{0, u, v}^{(p)}, \ldots, B_{p, u, v}^{(p)}\right\} .
$$

This basis can be constructed by imposing that the basis elements sum up to 1 $(p \geq 2)$ and that they behave like Bernstein polynomials at the two end points of $I=[a, b]$, see (7) and (8). More precisely, see [17],

$$
\begin{aligned}
& \frac{\mathrm{d}^{r} B_{i, u, v}^{(p)}(a)}{\mathrm{d} r^{r}}=0, \quad r=0, \ldots, i-1 ; \\
& \frac{\mathrm{d}^{r} B_{i, u, v}^{(p)}(b)}{\mathrm{d} t^{r}}=0, \quad r=0, \ldots, p-i-1 .
\end{aligned}
$$

Figure 20 illustrates the ONTP basis of $\mathbb{P}_{3}^{u, v}$ for different choices of $\langle u, v\rangle$.

- Recurrence relation. With assumption (67), there exists a unique element in $\langle U, V\rangle$ which takes the values 0 and 1 ( 1 and 0$)$ at the two end points of the interval $I$. Moreover, such an element has no other roots in the interval, so it is positive on $I$. Therefore, by setting $I=[0,1]$, without loss of generality we may assume

$$
U(0)=1, U(1)=0, \quad V(0)=0, V(1)=1 .
$$

Then, by taking

$$
B_{0, u, v}^{(1)}:=U, \quad B_{1, u, v}^{(1)}:=V
$$

in a complete analogy with (11), we have (see [17])

$$
\begin{aligned}
B_{0, u, v}^{(p)}(t) & :=1-d_{0, u, v}^{(p-1)} \int_{0}^{t} B_{0, u, v}^{(p-1)}(s) \mathrm{d} s \\
B_{i, u, v}^{(p)}(t) & :=d_{i-1, u, v}^{(p-1)} \int_{0}^{t} B_{i-1, u, v}^{(p-1)}(s) \mathrm{d} s-d_{i, u, v}^{(p-1)} \int_{0}^{t} B_{i, u, v}^{(p-1)}(s) \mathrm{d} s, \quad i=1, \ldots, p-1, \\
B_{p, u, v}^{(p)}(t) & :=d_{p-1, u, v}^{(p-1)} \int_{0}^{t} B_{p-1, u, v}^{(p-1)}(s) \mathrm{d} s
\end{aligned}
$$

where

$$
d_{i, u, v}^{(p)}:=\frac{1}{\int_{0}^{1} B_{i, u, v}^{(p)}(s) \mathrm{d} s} .
$$

- Degree elevation. Since $\mathbb{P}_{p}^{u, v} \subset \mathbb{P}_{p+1}^{u, v}$, in a complete analogy with (16), we have (see [17])

$$
\sum_{i=0}^{p} \mathbf{P}_{i} B_{i, u, v}^{(p)}(t)=\sum_{i=0}^{p+1} \hat{\mathbf{P}}_{i} B_{i, u, v}^{(p+1)}(t)
$$

where

$$
\hat{\mathbf{P}}_{0}:=\mathbf{P}_{0}, \quad \hat{\mathbf{P}}_{i}:=\theta_{i} \mathbf{P}_{i}+\left(1-\theta_{i}\right) \mathbf{P}_{i-1}, \quad \hat{\mathbf{P}}_{p+1}:=\mathbf{P}_{p},
$$


and

$$
\theta_{i}:=\frac{\frac{\mathrm{d}^{i}}{\mathrm{~d} t^{i}} B_{i, u, v}^{(p)}(0)}{\frac{\mathrm{d}^{i}}{\mathrm{~d} t^{i}} B_{i, u, v}^{(p+1)}(0)}, \quad i=1, \ldots, p
$$

Example 8. The most popular choices for spaces $\mathbb{P}_{p}^{u, v}$ are

$$
\begin{aligned}
& \mathbb{P}_{p}^{u, v}:=\left\langle 1, \ldots, t^{p-2}, \cos \omega t, \sin \omega t\right\rangle, \\
& \mathbb{P}_{p}^{u, v}:=\left\langle 1, \ldots, t^{p-2}, \cosh \omega t, \sinh \omega t\right\rangle .
\end{aligned}
$$

Figure 21 illustrates some starting elements (68) for spaces of the form (69)-(70). In such a case, assuming $I=[a, b]$, we have

- $\langle u, v\rangle=\langle\cos \omega t, \sin \omega t\rangle, 0<\omega(b-a)<\pi$,

- $\langle u, v\rangle=\langle\cosh \omega t, \sinh \omega t\rangle, 0<\omega$.

\subsection{Generalized B-Splines}

We now consider spaces of functions belonging piecewisely to $\mathbb{P}_{p}^{u_{i}, v_{i}}$, see (66), and with some prescribed smoothness conditions. For these spaces it is possible to construct a basis with the same properties as B-splines, the so-called generalized $B$ splines ( $G B$-splines).

Let the knots $\Xi:=\left\{\xi_{1} \leq \xi_{2} \leq \ldots \leq \xi_{n+p+1}\right\}$ be given. Although more general constructions can be obtained with less restrictive hypotheses, a neat theory of GBsplines can be presented by assuming $u_{i}, v_{i} \in C^{p}\left[\xi_{i}, \xi_{i+1}\right]$ and

$$
\left\langle U_{i}(t), V_{i}(t)\right\rangle, t \in\left[\xi_{i}, \xi_{i+1}\right], \text { is an EC space on } I,
$$

where

$$
U_{i}:=\frac{\mathrm{d}^{p-1}}{\mathrm{~d} t^{p-1}} u_{i}, \quad V_{i}:=\frac{\mathrm{d}^{p-1}}{\mathrm{~d} t^{p-1}} v_{i} .
$$

Thus, as in the previous subsection, without loss of generality, we may assume

$$
U_{i}\left(\xi_{i}\right)=1, U_{i}\left(\xi_{i+1}\right)=0, \quad V_{i}\left(\xi_{i}\right)=0, V_{i}\left(\xi_{i+1}\right)=1 .
$$

According to [38], see also [37, 41, 46, 68] and the references therein, generalized $\mathrm{B}$-splines of a certain degree ${ }^{20}$ can be defined by a recurrence relation completely similar $^{21}$ to the classical polynomial case, see (47).

\footnotetext{
${ }^{20}$ We use the term degree to stress the similarity with classical polynomial B-splines.

21 The generalized B-splines are given by successive integration of elements in the bidimensional spaces (71). Therefore, it is natural to start the recurrence relation from degree 1, instead of from degree 0 as is usually done in the polynomial case (see Definition 6).
} 

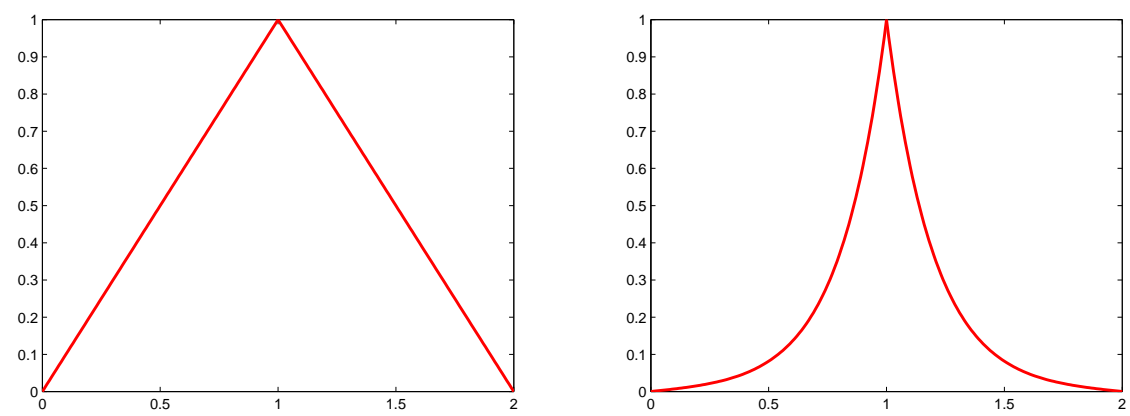

Fig. 22 Left: polynomial B-spline $B_{i, \Xi}^{(1)}$ of degree 1. Right: exponential B-spline $\widehat{B}_{i, \Xi}^{(1)}$ of degree 1 with $\left\langle u_{i}, v_{i}\right\rangle=\langle\cosh (5 t), \sinh (5 t)\rangle$.
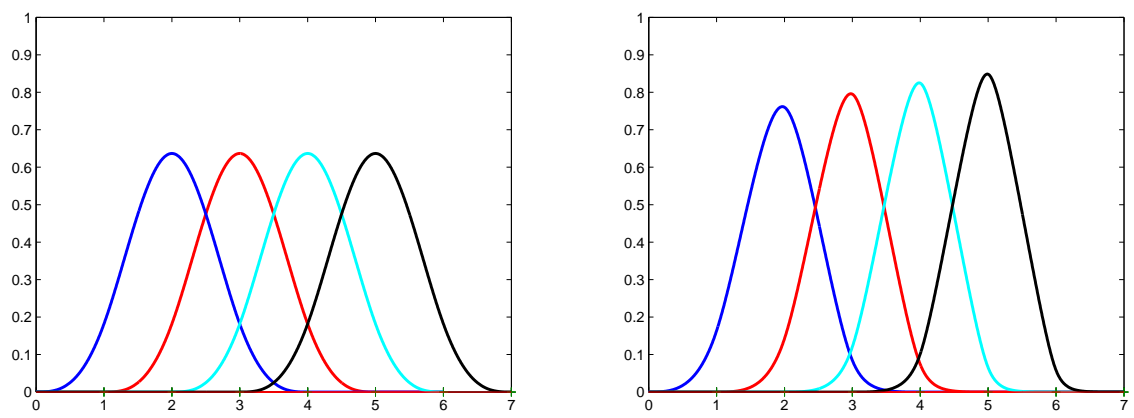

Fig. 23 Generalized cubic B-splines. Left: $\left\langle u_{i}, v_{i}\right\rangle=\left\langle\cos \left(\frac{\pi}{2} t\right), \sin \left(\frac{\pi}{2} t\right)\right\rangle$. Right: $\left\langle u_{i}, v_{i}\right\rangle=$ $\langle\cosh (i t), \sinh (i t)\rangle$.

Definition 11. Generalized B-splines $\widehat{B}_{i, \Xi}^{(p)}$ of degree p, related to the knots $\Xi$, are given by

$$
\begin{aligned}
& \widehat{B}_{i, \Xi}^{(1)}(t):= \begin{cases}V_{i}(t), & \text { if } t \in\left[\xi_{i}, \xi_{i+1}\right), \\
U_{i+1}(t), & \text { if } t \in\left[\xi_{i+1}, \xi_{i+2}\right), \\
0, & \text { elsewhere, }\end{cases} \\
& \widehat{B}_{i, \Xi}^{(p)}(t):=\widehat{d}_{i, \Xi}^{(p-1)} \int_{-\infty}^{t} \widehat{B}_{i, \Xi}^{(p-1)}(s) \mathrm{d} s-\widehat{d}_{i+1, \Xi}^{(p-1)} \int_{-\infty}^{t} \widehat{B}_{i+1, \Xi}^{(p-1)}(s) \mathrm{d} s,
\end{aligned}
$$

where

$$
\widehat{d}_{i, \Xi}^{(p)}:=\frac{1}{\int_{-\infty}^{+\infty} \widehat{B}_{i, \Xi}^{(p)}(s) \mathrm{d} s} .
$$

Figure 22 illustrates some starting elements (72) for the recurrence relation in the case of GB-splines. Some cubic GB-spline bases are depicted in Fig. 23. 
Example 9. GB-splines of salient interest are those with section spaces (69) and (70). We will refer to them as trigonometric GB-splines and exponential GB-splines, respectively, see $[68,70]$.

GB-splines of degree $p \geq 2$ possess all fundamental properties of classical Bsplines.

- Piecewise structure: $\widehat{B}_{i, \Xi}^{(p)}(t) \in \mathbb{P}_{p}^{u_{j}, v_{j}}, t \in\left[\xi_{j}, \xi_{j+1}\right)$.

- Positivity.

- Partition of unity.

- Compact support.

- Smoothness depending on knot multiplicity.

- Local linear independence.

- Total positivity.

Moreover, derivative formulas similar to (45) can be obtained from (73).

Remark 13. We note that the elements in the space spanned by GB-splines usually possess shape properties in the sense that the section spaces $\mathbb{P}_{p}^{u_{i}, v_{i}}$ are often equipped with parameters which can be used to control the shape of the obtained curves.

Finally, as far as trigonometric GB-splines and exponential GB-splines are concerned, we remark that

- they naturally allow an exact representation of arcs of conic sections with respect to the arc-length;

- algebraic, trigonometric and exponential parts can be used in consecutive intervals to describe very general profiles;

- trigonometric and exponential GB-splines approach B-splines as the parameters $\omega_{i}$ approach 0 .

Remark 14. GB-splines with sections in EC spaces possess all fundamental properties for design as B-splines, see [48]. In particular, corner cutting algorithms can be used for evaluation. Evaluation algorithms specially tuned for trigonometric and exponential GB-splines have been proposed by several authors, see for example $[10,42]$ and references therein. Stable evaluation can also be obtained by means of non-stationary subdivision [24].

\section{Beyond Tensor-Product}

As we have seen in Sect. 3.5, tensor-product structures provide an easy way to extend a univariate setting to a bivariate (multivariate) one. However, the simplicity of the structure prevents to do local refinement. On the other hand, working with triangulations is a very natural way to deal with local mesh refinements. In this section, we describe how to define Bernstein polynomials on triangles and B-splines on suitable triangulations, with a special focus on Powell-Sabin B-splines. 


\subsection{Barycentric Coordinates}

Let $T:=\left\langle\mathbf{V}_{1}, \mathbf{V}_{2}, \mathbf{V}_{3}\right\rangle$ be a nondegenerate triangle in $\mathbb{R}^{2}$ with $\mathbf{V}_{i}:=\left(V_{i, x}, V_{i, y}\right)$.

Definition 12. Any point $\mathbf{X} \in \mathbb{R}^{2}$ can be uniquely expressed in terms of its barycentric coordinates $\left(\tau_{1}, \tau_{2}, \tau_{3}\right)$ with respect to the triangle $T$, such that

$$
\mathbf{X}=\tau_{1} \mathbf{V}_{1}+\tau_{2} \mathbf{V}_{2}+\tau_{3} \mathbf{V}_{3},
$$

and

$$
1=\tau_{1}+\tau_{2}+\tau_{3}
$$

If the point $\mathbf{X}$ lies inside $T$, then its barycentric coordinates are all positive. The barycentric coordinates of the three vertices $\mathbf{V}_{1}, \mathbf{V}_{2}$ and $\mathbf{V}_{3}$ are $(1,0,0),(0,1,0)$ and $(0,0,1)$, respectively.

The barycentric coordinates of the point $\mathbf{X}$ with respect to $T$ have a clear geometric interpretation. For instance, $\tau_{1}$ is the ratio between the signed area of the subtriangle $T_{1}:=\left\langle\mathbf{X}, \mathbf{V}_{2}, \mathbf{V}_{3}\right\rangle$ and the signed area of the triangle $T$. A similar relation holds for $\tau_{2}$ and $\tau_{3}$, using the signed areas of the subtriangles $T_{2}:=\left\langle\mathbf{X}, \mathbf{V}_{3}, \mathbf{V}_{1}\right\rangle$ and $T_{3}:=\left\langle\mathbf{X}, \mathbf{V}_{1}, \mathbf{V}_{2}\right\rangle$, see Fig. 24 .

Fig. 24 Geometric interpretation of barycentric coordi-

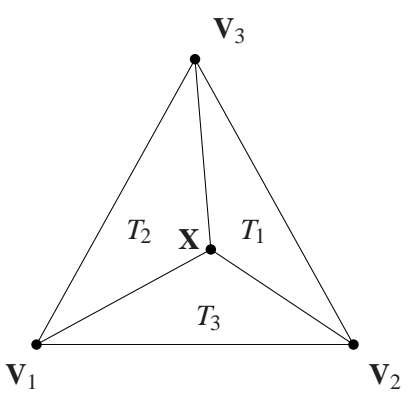
nates.

Definition 13. Given two points $\mathbf{X}_{1}$ and $\mathbf{X}_{2}$ in $\mathbb{R}^{2}$, the barycentric directional coordinates $\left(\delta_{1}, \delta_{2}, \delta_{3}\right)$ of the vector $\mathbf{X}_{2}-\mathbf{X}_{1}$ with respect to $T$ are defined as the difference of the barycentric coordinates of both points.

Example 10. The barycentric directional coordinates of the unit vectors $\mathbf{x}$ and $\mathbf{y}$ in the $x$ - and $y$-direction, respectively, are given by

$$
\begin{aligned}
& \left(\delta_{1}^{x}, \delta_{2}^{x}, \delta_{3}^{x}\right):=\left(V_{2, y}-V_{3, y}, V_{3, y}-V_{1, y}, V_{1, y}-V_{2, y}\right) / E, \\
& \left(\delta_{1}^{y}, \delta_{2}^{y}, \delta_{3}^{y}\right):=\left(V_{3, x}-V_{2, x}, V_{1, x}-V_{3, x}, V_{2, x}-V_{1, x}\right) / E,
\end{aligned}
$$

with

$$
E:=\left|\begin{array}{ccc}
1 & 1 & 1 \\
V_{1, x} & V_{2, x} & V_{3, x} \\
V_{1, y} & V_{2, y} & V_{3, y}
\end{array}\right| .
$$



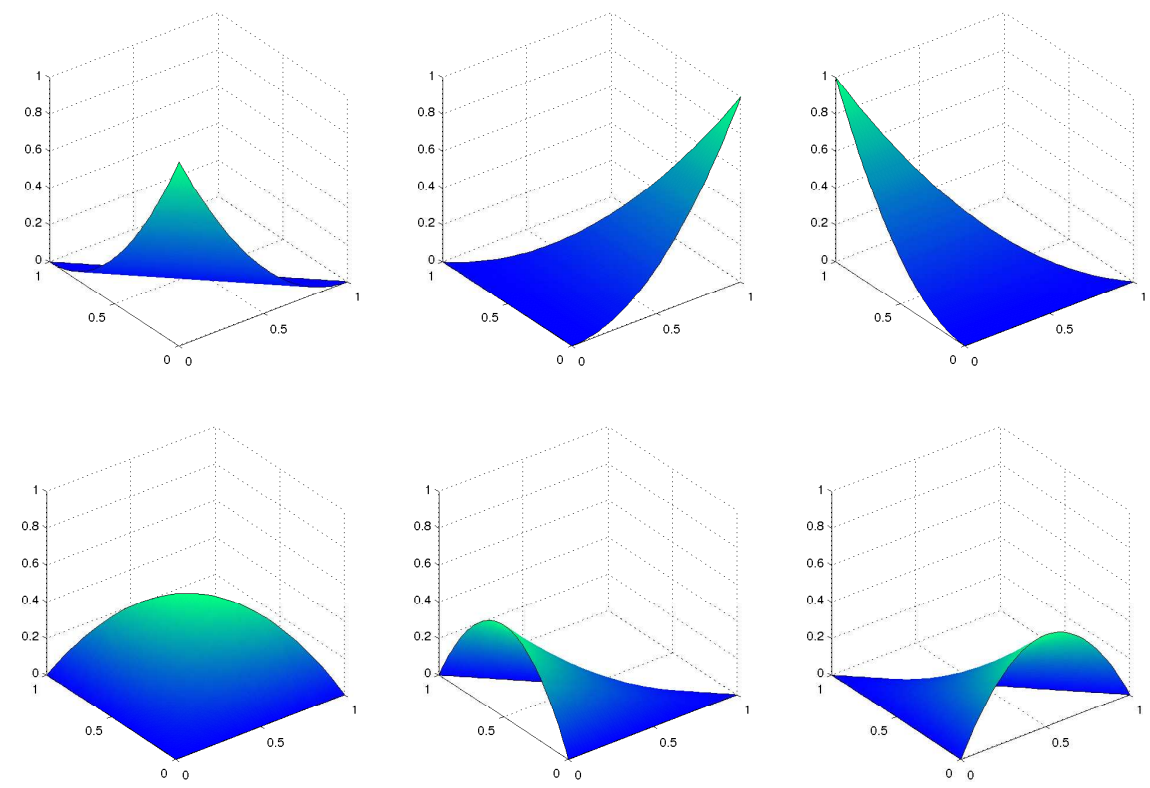

Fig. 25 Quadratic triangular Bernstein polynomials.

\subsection{Triangular Bernstein Polynomials}

In this subsection we introduce triangular Bernstein polynomials and we present their most popular algebraic and geometric properties. The results are completely analogous with the ones described in Sect. 2.1 devoted to univariate Bernstein polynomials.

\subsubsection{Definition and Main Properties}

Definition 14. Triangular Bernstein polynomials ${ }^{22}$ of degree $p$ are given by

$$
B_{i, j, k}^{(p)}(\mathbf{X}):=\frac{p !}{i ! j ! k !} \tau_{1}{ }^{i} \tau_{2}{ }^{j} \tau_{3}{ }^{k}, \quad \forall i+j+k=p,
$$

with $\left(\tau_{1}, \tau_{2}, \tau_{3}\right)$ the barycentric coordinates of $\mathbf{X}$.

For a given triangle, Fig. 25 collects the six triangular Bernstein polynomials of degree 2, and Fig. 26 shows the ten Bernstein polynomials of degree 3.

\footnotetext{
${ }^{22}$ Along each edge of the triangle $T$, the triangular Bernstein polynomials reduce to the univariate Bernstein polynomials (1).
} 

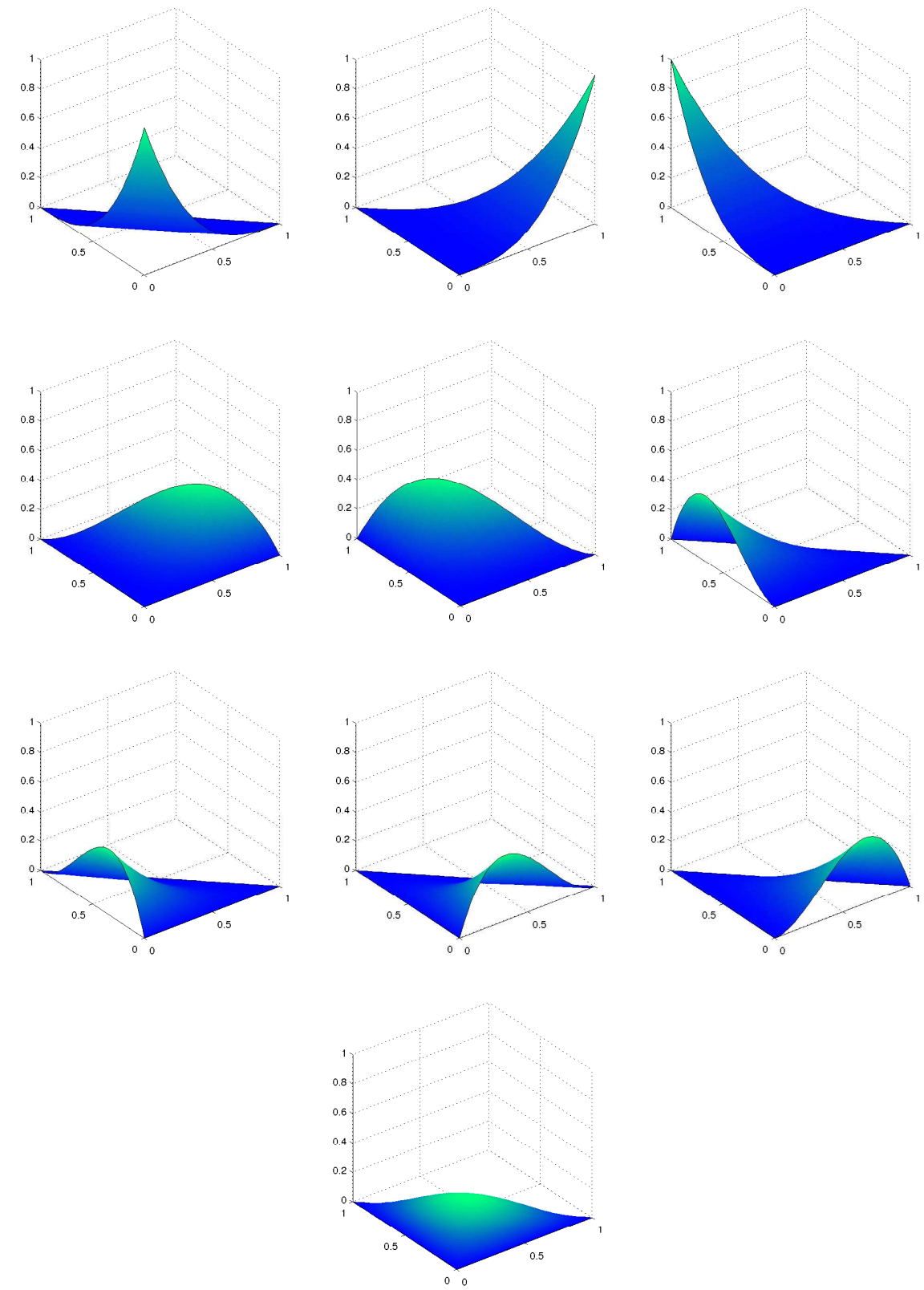

Fig. 26 Cubic triangular Bernstein polynomials. 
The Bernstein polynomials form a basis for the bivariate space of algebraic polynomials $^{23}$, i.e., any $q(\mathbf{X}) \in \mathbb{P}_{p}$ has a unique representation

$$
q(\mathbf{X})=\sum_{i+j+k=p} q_{i, j, k} B_{i, j, k}^{(p)}(\mathbf{X}) .
$$

We refer to $q_{i, j, k}$ as the Bézier ordinates of $q(\mathbf{X})$. Note that the dimension of $\mathbb{P}_{p}$ is equal to $\left(\begin{array}{c}p+2 \\ 2\end{array}\right)$. The triangular Bernstein polynomials possess properties very similar to the univariate case, see Sect. 2.1.1.

- Positivity.

$$
B_{i, j, k}^{(p)}(\mathbf{X}) \geq 0, \quad \mathbf{X} \in T
$$

- Partition of unity.

$$
\sum_{i+j+k=p} B_{i, j, k}^{(p)}(\mathbf{X})=\left(\tau_{1}+\tau_{2}+\tau_{3}\right)^{p}=1, \quad \forall \mathbf{X} \in \mathbb{R}^{2} .
$$

- Recurrence relation.

$$
B_{i, j, k}^{(p)}(\mathbf{X})=\tau_{1} B_{i-1, j, k}^{(p-1)}(\mathbf{X})+\tau_{2} B_{i, j-1, k}^{(p-1)}(\mathbf{X})+\tau_{3} B_{i, j, k-1}^{(p-1)}(\mathbf{X}),
$$

with $B_{i, j, k}^{(l)}=0$ if $i<0$ or $j<0$ or $k<0$.

- Degree elevation.

$$
\begin{aligned}
B_{i, j, k}^{(p)}(\mathbf{X}) & =\left(\tau_{1}+\tau_{2}+\tau_{3}\right) B_{i, j, k}^{(p)}(\mathbf{X}) \\
& =\frac{i+1}{p+1} B_{i+1, j, k}^{(p+1)}(\mathbf{X})+\frac{j+1}{p+1} B_{i, j+1, k}^{(p+1)}(\mathbf{X})+\frac{k+1}{p+1} B_{i, j, k+1}^{(p+1)}(\mathbf{X})
\end{aligned}
$$

- Directional derivatives. Let $\mathbf{u}$ be a vector in $\mathbb{R}^{2}$ and let $\left(\delta_{1}, \delta_{2}, \delta_{3}\right)$ be its barycentric directional coordinates with respect to the triangle $T$.

- first derivative:

$$
D_{\mathbf{u}} B_{i, j, k}^{(p)}(\mathbf{X})=p\left[\delta_{1} B_{i-1, j, k}^{(p-1)}(\mathbf{X})+\delta_{2} B_{i, j-1, k}^{(p-1)}(\mathbf{X})+\delta_{3} B_{i, j, k-1}^{(p-1)}(\mathbf{X})\right]
$$

- end derivatives:

$$
\begin{array}{ll}
D_{\mathbf{u}}^{r} B_{i, j, k}^{(p)}\left(\mathbf{V}_{1}\right)=0, & r=0, \ldots, p-i-1 ; \\
D_{\mathbf{u}}^{r} B_{i, j, k}^{(p)}\left(\mathbf{V}_{2}\right)=0, & r=0, \ldots, p-j-1 ; \\
D_{\mathbf{u}}^{r} B_{i, j, k}^{(p)}\left(\mathbf{V}_{3}\right)=0, & r=0, \ldots, p-k-1 ;
\end{array}
$$

${ }^{23}$ In both the univariate and bivariate case we use the same symbol for the space of algebraic polynomials, $\mathbb{P}_{p}$, but the meaning will be clear from the context. 
- unimodal behavior: let $i \geq 1, j \geq 1, k \geq 1$, it holds that

$$
D_{\mathbf{u}} B_{i, j, k}^{(p)}(\mathbf{X})=0
$$

for any direction $\mathbf{u}$ and for a point $\mathbf{X}$ in the interior of $T$, if and only if

$$
\mathbf{X}=\frac{i \mathbf{V}_{1}+j \mathbf{V}_{2}+k \mathbf{V} 3}{p}=: \boldsymbol{\xi}_{i, j, k}^{*}
$$

- Integration.

$$
\begin{aligned}
& \int_{T} B_{i, j, k}^{(p)}(\mathbf{X}) \mathrm{d} \mathbf{X}=\frac{|E|}{2\left(\begin{array}{c}
p+2 \\
2
\end{array}\right)}, \\
& \int_{T} B_{i_{1}, j_{1}, k_{1}}^{(p)}(\mathbf{X}) B_{i_{2}, j_{2}, k_{2}}^{(p)}(\mathbf{X}) \mathrm{d} \mathbf{X}=\frac{\left(\begin{array}{c}
i_{1}+i_{2} \\
i_{1}
\end{array}\right)\left(\begin{array}{c}
j_{1}+j_{2} \\
j_{1}
\end{array}\right)\left(\begin{array}{c}
k_{1}+k_{2} \\
k_{1}
\end{array}\right)|E|}{2\left(\begin{array}{c}
2 p \\
p
\end{array}\right)\left(\begin{array}{c}
2 p+2 \\
2
\end{array}\right)},
\end{aligned}
$$

where $E$ is defined in (76). Note that $|E| / 2$ is the area of the triangle $T$.

For more details, we refer to [34, 54] or to [39] for a deeper insight.

Example 11. Considering $q(\mathbf{X})=\mathbf{X}$, we have

$$
\mathbf{X}=\sum_{i+j+k=p} \boldsymbol{\xi}_{i, j, k}^{*} B_{i, j, k}^{(p)}(\mathbf{X}) .
$$

The points $\boldsymbol{\xi}_{i, j, k}^{*}$, defined in (88), are called Greville abscissas or domain points. The set of all these points is denoted by $\mathcal{D}_{p, T}$. They are uniformly spaced over the triangle $T$, and they can be triangulated by connecting each pair of two domain points $\boldsymbol{\xi}_{i_{1}, j_{1}, k_{1}}^{*}$ and $\boldsymbol{\xi}_{i_{2}, j_{2}, k_{2}}^{*}$ provided that

$$
\left|i_{1}-i_{2}\right|+\left|j_{1}-j_{2}\right|+\left|k_{1}-k_{2}\right|=2,
$$

see Fig. 27 (left) for the quadratic case.

\subsubsection{Geometrical and Graphical Properties}

The above properties lead again to a number of interesting geometric consequences. Let $\mathbf{P}_{i, j, k} \in \mathbb{R}^{d}, i+j+k=p$, be given. The parametric surface

$$
\mathcal{S}(\mathbf{X}):=\sum_{i+j+k=p} \mathbf{P}_{i, j, k} B_{i, j, k}^{(p)}(\mathbf{X})
$$

is called a Bézier surface, and the points $\mathbf{P}_{i, j, k}$ are its control points. The piecewise linear interpolant connecting these points as given by (92) is called the control net of $\mathcal{S}(\mathbf{X})$. The graph of any polynomial $q(\mathbf{X})$ in (78) can be seen as a Bézier surface, namely 

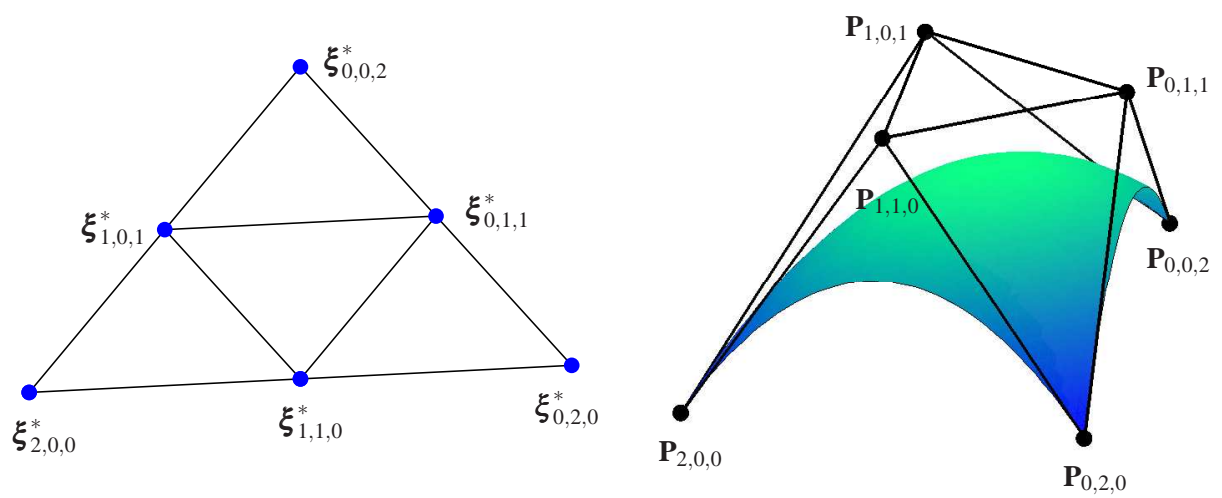

Fig. 27 Left: quadratic domain points. Right: control net of a quadratic polynomial.

$$
\left(\begin{array}{c}
\mathbf{X} \\
q(\mathbf{X})
\end{array}\right)=\sum_{i+j+k=p}\left(\begin{array}{c}
\boldsymbol{\xi}_{i, j, k}^{*} \\
q_{i, j, k}
\end{array}\right) B_{i, j, k}^{(p)}(\mathbf{X})
$$

and the piecewise linear interpolant connecting $\left(\begin{array}{c}\boldsymbol{\xi}_{i, j, k}^{*} \\ q_{i, j, k}\end{array}\right)$ is its control net. Figure 27 (right) illustrates the control net of a quadratic Bézier surface.

The geometric properties of Bézier curves, see Sect. 2.1.2, extend to the triangular case as follows.

- Convex hull property. The positivity and partition of unity imply that $\mathcal{S}(\mathbf{X}) \in$ $\mathcal{H}\left(\mathbf{P}_{i, j, k}, i+j+k=p\right)$ for $\mathbf{X} \in T$.

- Triangular de Casteljau algorithm. The recurrence relation (81) leads to a stable evaluation algorithm, a triangular variant of the de Casteljau algorithm:

$$
\begin{aligned}
\mathcal{S}(\mathbf{X}) & =\sum_{i+j+k=p} \mathbf{P}_{i, j, k}^{[p]} B_{i, j, k}^{(p)}(\mathbf{X}) \\
& =\sum_{i+j+k=p} \mathbf{P}_{i, j, k}^{[p]}\left[\tau_{1} B_{i-1, j, k}^{(p-1)}(\mathbf{X})+\tau_{2} B_{i, j-1, k}^{(p-1)}(\mathbf{X})+\tau_{3} B_{i, j, k-1}^{(p-1)}(\mathbf{X})\right] \\
& =\sum_{i+j+k=p-1} \mathbf{P}_{i, j, k}^{[p-1]} B_{i, j, k}^{(p-1)}(\mathbf{X})=\ldots=\mathbf{P}_{0,0,0}^{[0]},
\end{aligned}
$$

with $\left(\tau_{1}, \tau_{2}, \tau_{3}\right)$ the barycentric coordinates of $\mathbf{X}$, and

$$
\mathbf{P}_{i, j, k}^{[l-1]}:=\tau_{1} \mathbf{P}_{i+1, j, k}^{[l]}+\tau_{2} \mathbf{P}_{i, j+1, k}^{[l]}+\tau_{3} \mathbf{P}_{i, j, k+1}^{[l]}, \quad i+j+k=l-1 .
$$

This algorithm provides a very efficient tool to evaluate a Bézier surface at a given parameter value $\mathbf{X} \in T$. 
- Degree elevation. By using (82), and by setting $\mathbf{P}_{i, j, k}=0$ if $i<0$ or $j<0$ or $k<0$, we obtain

$$
\sum_{i+j+k=p} \mathbf{P}_{i, j, k} B_{i, j, k}^{(p)}(\mathbf{X})=\sum_{i+j+k=p+1} \hat{\mathbf{P}}_{i, j, k} B_{i, j, k}^{(p+1)}(\mathbf{X}),
$$

with

$$
\hat{\mathbf{P}}_{i, j, k}:=\frac{i}{p+1} \mathbf{P}_{i-1, j, k}+\frac{j}{p+1} \mathbf{P}_{i, j-1, k}+\frac{k}{p+1} \mathbf{P}_{i, j, k-1} .
$$

- Directional derivatives. Let $\mathbf{u}$ be a vector in $\mathbb{R}^{2}$ and let $\left(\delta_{1}, \delta_{2}, \delta_{3}\right)$ be its barycentric directional coordinates with respect to the triangle $T$. Directional derivatives of Bézier surfaces are obtained by applying (83):

$$
D_{\mathbf{u}} \mathcal{S}(\mathbf{X})=p \sum_{i+j+k=p-1}\left[\delta_{1} \mathbf{P}_{i+1, j, k}+\delta_{2} \mathbf{P}_{i, j+1, k}+\delta_{3} \mathbf{P}_{i, j, k+1}\right] B_{i, j, k}^{(p-1)}(\mathbf{X}) .
$$

More generally, the $r$-th order directional derivative is given by

$$
D_{\mathbf{u}}^{r} \mathcal{S}(\mathbf{X})=\frac{p !}{(p-r) !} \sum_{i+j+k=p-r} \Delta_{r}\left(\mathbf{P}_{i, j, k}\right) B_{i, j, k}^{(p-r)}(\mathbf{X}), \quad p \geq r
$$

where $\Delta_{r}\left(\mathbf{P}_{i, j, k}\right):=\mathbf{P}_{i, j, k}^{[p-r]}$ are the quantities (93) obtained after $r$ steps of the triangular de Casteljau algorithm applied to the control points of $\mathcal{S}(\mathbf{X})$ using the barycentric directional coordinates of $\mathbf{u}$.

- Derivatives at vertices. From (95) it follows that the derivative at the vertex $\mathbf{V}_{1}$ can be written as

$$
D_{\mathbf{u}} \mathcal{S}\left(\mathbf{V}_{1}\right)=p\left[\delta_{1} \mathbf{P}_{p, 0,0}+\delta_{2} \mathbf{P}_{p-1,1,0}+\delta_{3} \mathbf{P}_{p-1,0,1}\right],
$$

implying that the control net is tangent to the surface at the three corner points. More generally, $\left(\begin{array}{c}r+2 \\ 2\end{array}\right)$ control points are involved in the expression of $D_{\mathbf{u}}^{r} \mathcal{S}\left(\mathbf{V}_{i}\right)$.

- Smooth joints of Bézier patches. $C^{r}$ continuity between two adjacent Bézier surfaces

$$
\sum_{i+j+k=p} \mathbf{P}_{i, j, k}^{L} B_{i, j, k}^{(p)}(\mathbf{X}), \quad \sum_{i+j+k=p} \mathbf{P}_{i, j, k}^{R} B_{i, j, k}^{(p)}(\mathbf{X}),
$$

defined on the triangles $T_{L}:=\left\langle\mathbf{V}_{1}, \mathbf{V}_{2}, \mathbf{V}_{3}\right\rangle$ and $T_{R}:=\left\langle\mathbf{V}_{1}, \mathbf{V}_{2}, \mathbf{V}_{4}\right\rangle$ respectively, has a simple geometric interpretation thanks to the local behavior of the derivatives at the end points, see (95) and (96). In particular, $C^{0}$ continuity just requires that $\mathbf{P}_{i, j, 0}^{L}=\mathbf{P}_{i, j, 0}^{R}$ for all $i+j=p$, while $C^{1}$ continuity implies, in addition, that for each $i+j=p-1$ the pair of triangles

$$
\left\langle\mathbf{P}_{i, j, 1}^{L}, \mathbf{P}_{i+1, j, 0}^{L}, \mathbf{P}_{i, j+1,0}^{L}\right\rangle, \quad\left\langle\mathbf{P}_{i, j, 1}^{R}, \mathbf{P}_{i+1, j, 0}^{R}, \mathbf{P}_{i, j+1,0}^{R}\right\rangle
$$

are coplanar. In Fig. 28 two quadratic Bézier patches are depicted with a $C^{1}$ smooth joint. 


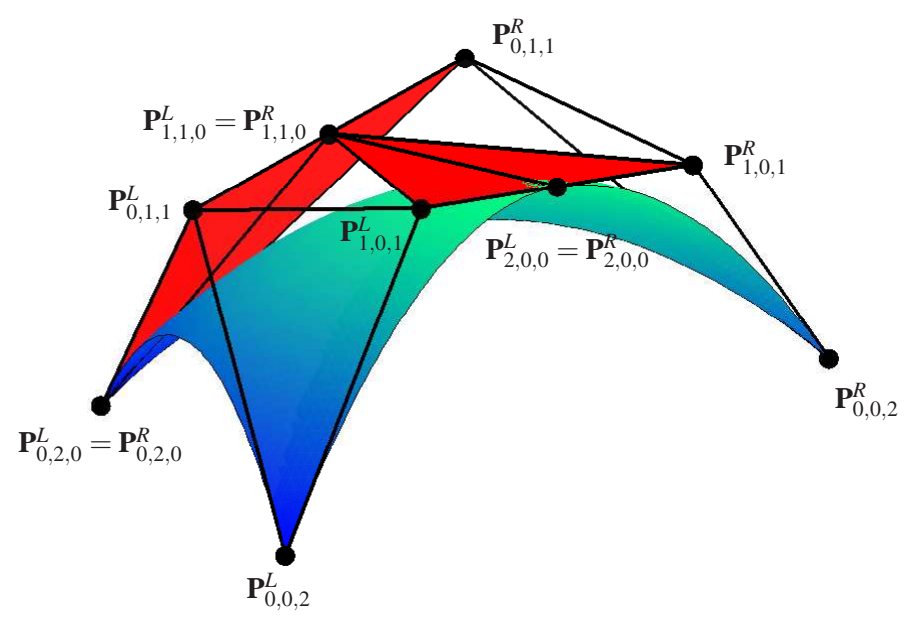

Fig. $28 C^{1}$ joint between two quadratic Bézier surfaces.

Remark 15. So far we have seen a complete analogy between bivariate triangular and univariate Bernstein polynomials and the related representations. Nevertheless, there are some fundamental differences between them. For instance, it is not clear whether there is a geometric variation diminishing property beyond the univariate case [55], and also the concept of total positivity has not been properly generalized yet, see [39, Conjecture 2.22].

\subsection{Splines on Triangulations}

There exist different extensions to the bivariate (multivariate) setting of the concept of spline spaces and B-spline functions [9]. A natural way to extend to the bivariate setting the structure of univariate splines is to define piecewise polynomials with a suitable smoothness on a triangulation [39].

Definition 15. A triangulation $\mathcal{T}:=\left\{T_{i}, i=1, \ldots, N_{t}\right\}$ of a polygonal set $\Omega \subseteq$ $\mathbb{R}^{2}$ is a partition of $\Omega$ consisting of non-overlapping triangles. No triangle $T_{i} \in \mathcal{T}$ contains a vertex of any other triangle $T_{j} \in \mathcal{T}$ that is different from its own three vertices.

Definition 16. Given a triangulation $\mathcal{T}$ of $\Omega$, the spline space of degree $p$ on $\mathcal{T}$ with $C^{r}$ continuity is defined as

$$
\mathbb{S}_{p, \mathcal{T}}^{r}:=\left\{s \in C^{r}(\Omega):\left.s\right|_{T_{i}} \in \mathbb{P}_{p}, T_{i} \in \mathcal{T}\right\}
$$

As we have seen, it is convenient to represent polynomials on triangles in their Bézier-Bernstein form. We can use this form for the characterization of splines de- 
fined on triangulations. The set of domain points of degree $p$ associated to the triangulation $\mathcal{T}$ is denoted by

$$
\mathcal{D}_{p, \mathcal{T}}:=\bigcup_{T_{i} \in \mathcal{T}} \mathcal{D}_{p, T_{i}}
$$

i.e. the union of all domain points (88) on each triangle $T_{i} \in \mathcal{T}$. Figure 29 illustrates the set of quadratic domain points for a given triangulation.

Let $N_{t}, N_{v}$ and $N_{e}$ be the number of triangles, vertices and edges in $\mathcal{T}$. The dimension of $\mathbb{S}_{p, \mathcal{T}}^{0}$ is equal to the number of domain points in $\mathcal{D}_{p, \mathcal{T}}$, i.e.,

$$
\operatorname{dim}\left(\mathbb{S}_{p, \mathcal{T}}^{0}\right)=N_{v}+(p-1) N_{e}+\left(\begin{array}{c}
p-1 \\
2
\end{array}\right) N_{t}, \quad p \geq 1
$$

For any spline $s \in \mathbb{S}_{p, \mathcal{T}}^{0}$ there exists a unique set of coefficients $\left\{c_{\boldsymbol{\xi}}, \boldsymbol{\xi} \in \mathcal{D}_{p, \mathcal{T}}\right\}$ such that for each $T_{i} \in \mathcal{T}$,

$$
\left.s\right|_{T_{i}}=\left.\sum_{\boldsymbol{\xi} \in \mathcal{D}_{p, T_{i}}} c_{\boldsymbol{\xi}} B_{\boldsymbol{\xi}, T_{i}}^{(p)}\right|_{T_{i}}
$$

where $B_{\boldsymbol{\xi}, T_{i}}^{(p)}$ is the Bernstein polynomial (77) of degree $p$ associated to the triangle $T_{i}$ and to the triple of indices defining $\boldsymbol{\xi}$ with respect to $T_{i}$, see (88). The set of functions $\left\{P_{\boldsymbol{\xi}, \mathcal{T}}^{(p)}(\mathbf{X}), \boldsymbol{\xi} \in \mathcal{D}_{p, \mathcal{T}}\right\}$ defined by

$$
P_{\boldsymbol{\xi}, \mathcal{T}}^{(p)}(\mathbf{X}):= \begin{cases}B_{\boldsymbol{\xi}, T_{i}}^{(p)}(\mathbf{X}), & \text { if } \boldsymbol{\xi} \in \mathcal{D}_{p, T_{i}}, \mathbf{X} \in T_{i}, T_{i} \in \mathcal{T} \\ 0, & \text { elsewhere }\end{cases}
$$

forms a basis for $\mathbb{S}_{p, \mathcal{T}}^{0}$, with the following properties.

- Positivity.

- Partition of unity.

- Compact support.

Remark 16. A common basis for $\mathbb{S}_{p, \mathcal{T}}^{0}$ in the finite element literature is a Lagrangetype basis $\left\{L_{\boldsymbol{\xi}, \mathcal{T}}^{(p)}(\mathbf{X}), \boldsymbol{\xi} \in \mathcal{D}_{p, \mathcal{T}}\right\}$. These basis functions are defined as the unique interpolant of the problem

$$
L_{\boldsymbol{\xi}, \mathcal{T}}^{(p)}(\boldsymbol{\eta})=\delta_{\boldsymbol{\xi}, \boldsymbol{\eta}}, \quad \text { for all } \boldsymbol{\eta} \in \mathcal{D}_{p, \mathcal{T}},
$$

where $\delta_{\boldsymbol{\xi}, \boldsymbol{\eta}}$ denotes the Kronecker symbol. These basis functions are not positive.

In IgA spline spaces of higher smoothness are of interest. Smoothness conditions on a spline $s \in \mathbb{S}_{p, \mathcal{T}}^{0}$ are just linear conditions on the vector $\mathbf{c}$ of coefficients of $s$, see (95)-(96). Thus, given a set of smoothness conditions $\mathfrak{S}$, there is a matrix $A^{\mathfrak{S}}$ so that

$$
\mathbb{S}_{p, \mathcal{T}}^{\mathfrak{S}}:=\left\{s \in \mathbb{S}_{p, \mathcal{T}}^{0}: A^{\mathfrak{S}} \mathbf{c}=0\right\}
$$



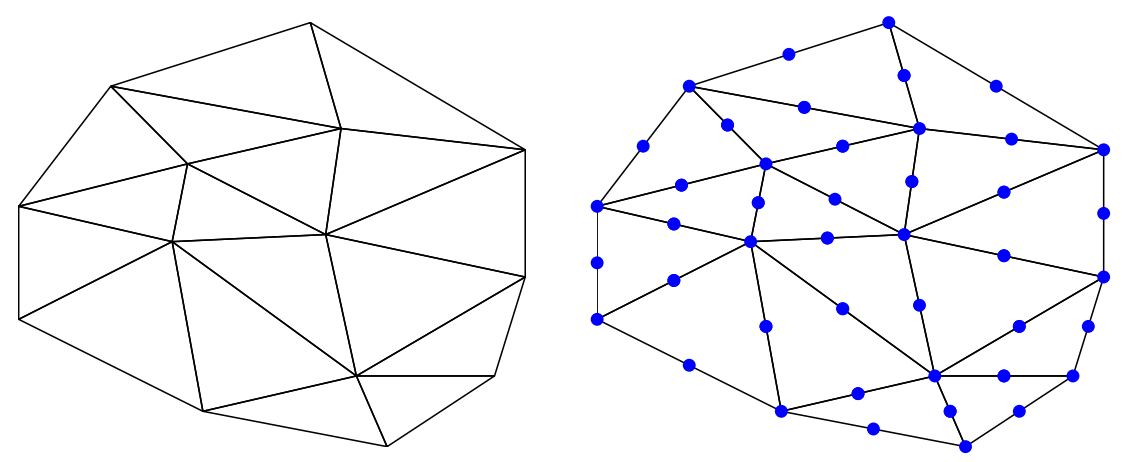

Fig. 29 A triangulation $\mathcal{T}$, and its set of quadratic domain points $\mathcal{D}_{2, \mathcal{T}}$.

The matrix $A^{\mathfrak{S}}$ is of size $m \times n$, with $m$ the number of smoothness conditions in $\mathfrak{S}$ and $n$ the dimension of $\mathbb{S}_{p, \mathcal{T}}^{0}$.

Theorem 15. Let $\mathbb{S}_{p, \mathcal{T}}^{\mathfrak{S}}$ be a smooth spline space as defined in (99), then the dimension of $\mathbb{S}_{p, \mathcal{T}}^{\mathfrak{S}}$ is equal to $n-k$, with $k$ the rank of the matrix $A^{\mathfrak{S}}$.

Remark 17. The computation of the dimension of smooth spline spaces defined on triangulations is a difficult task. There exist very sharp lower bounds and good upper bounds on the dimension of spline spaces for any combination of degree and smoothness, but there are still spaces for which no exact dimension formula is known, for instance the space $\mathbb{S}_{3, \mathcal{T}}^{1}$. We refer to [39] for a detailed discussion of the problem and an overview of smooth spline spaces on triangulations.

Remark 18. The dimension problem is linked to the construction of a so-called minimal determining set of domain points [39]. This is a subset $\mathcal{M} \subseteq \mathcal{D}_{p, \mathcal{T}}$ of minimal cardinality such that if $s \in \mathbb{S}_{p, \mathcal{T}}^{r}$ and $c_{\boldsymbol{\xi}}=0$ for all $\boldsymbol{\xi} \in \mathcal{M}$, then $s \equiv 0$. The cardinality of $\mathcal{M}$ is equal to the dimension of $\mathbb{S}_{p, \mathcal{T}}^{r}$.

Remark 19. The dimension of such spline spaces should be preferably expressed only in terms of geometrically interesting characteristics of the triangulation (like the number of vertices, edges, and triangles). Imposing additional local supersmoothness and/or considering triangulations with a particular macro-structure may simplify the dimension formula, and also the construction of a suitable basis (an example is elaborated in the next subsection).

Remark 20. Instead of defining first a particular spline space on a triangulation and then finding a suitable basis, one can also proceed the other way around: choose first a set of individual B-spline-like functions, and then define the spline space as the span of these functions. ${ }^{24}$ For example,

- box splines, see [7];

- simplex splines, see [21, 49, 50].

${ }^{24}$ In the univariate case both approaches lead to the same result, see Sect. 3.2. 

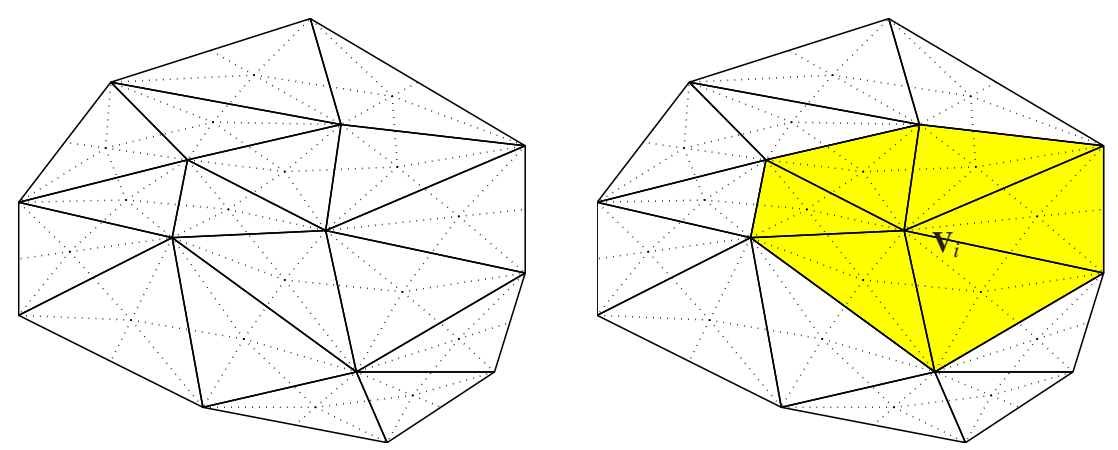

Fig. 30 Left: a PS refinement $\mathcal{T}^{*}$ of the triangulation $\mathcal{T}$ in Figure 29. Right: the molecule $\Omega_{i}$ of a vertex $\mathbf{V}_{i}$ of $\mathcal{T}$

\subsection{Powell-Sabin B-Splines}

In this subsection we focus on a spline space defined on triangulations with a specific macro-structure, the so-called Powell-Sabin (PS) spline space ${ }^{25}$. This space allows the definition of a suitable basis possessing the nice properties of univariate B-splines.

\subsubsection{Definition and Main Properties}

Definition 17. A Powell-Sabin refinement $\mathcal{T}^{*}$ of $\mathcal{T}$ is the refined triangulation $o b$ tained by subdividing each triangle of $\mathcal{T}$ into six smaller triangles as follows.

1. Select a point $\mathbf{Z}_{i}$ inside each triangle $T_{i}$ of $\mathcal{T}$ so that, if two triangles $T_{i}$ and $T_{j}$ have a common edge, then the line joining $\mathbf{Z}_{i}$ and $\mathbf{Z}_{j}$ intersects the common edge at a point $\mathbf{R}_{i, j}{ }^{26}$

2. Join each point $\mathbf{Z}_{i}$ to the vertices of $T_{i}$.

3. For each edge of the triangle $T_{i}$

a. which is common to a triangle $T_{j}$ : join $\mathbf{Z}_{i}$ to $\mathbf{R}_{i, j}$;

b. which belongs to the boundary $\partial \Omega$ : join $\mathbf{Z}_{i}$ to an arbitrary point $\mathbf{R}_{i, j}^{b}$ on that edge. $^{27}$

Figure 30 illustrates a possible PS refinement $\mathcal{T}^{*}$ of the triangulation $\mathcal{T}$ given in Figure 29.

${ }^{25}$ Powell-Sabin splines were originally developed in [53] with the main aim of drawing contour lines of bivariate functions.

${ }^{26}$ Such a choice is always possible: $\mathbf{Z}_{i}$ can be selected as the center of the inscribed circle. Usually, the barycenter of $T_{i}$ is also a valid choice (but not always).

${ }^{27}$ For boundary edges the subscript $j$ refers to the edge. 
Definition 18. Given a triangulation $\mathcal{T}$ of $\Omega$ and a PS refinement $\mathcal{T}^{*}$, the space of piecewise quadratic polynomials on $\mathcal{T}^{*}$ with global $C^{1}$ continuity is called the Powell-Sabin spline space:

$$
\mathbb{S}_{2, \mathcal{T}^{*}}^{1}:=\left\{s \in C^{1}(\Omega):\left.s\right|_{T_{i}^{*}} \in \mathbb{P}_{2}, T_{i}^{*} \in \mathcal{T}^{*}\right\}
$$

Let $\left\{\mathbf{V}_{k}, k=1, \ldots, N_{v}\right\}$ be the set of vertices of $\mathcal{T}$. It is known that the dimension of $\mathbb{S}_{2, \mathcal{T}^{*}}^{1}$ is equal to $3 N_{v}$.

Theorem 16. Given a set of triplets $\left(f_{k}, f_{x, k}, f_{y, k}\right)$ at the vertices $\mathbf{V}_{k}, k=1, \ldots, N_{v}$, there exists a unique Powell-Sabin spline $s \in \mathbb{S}_{2, \mathcal{T}^{*}}^{1}$ that satisfies

$$
s\left(\mathbf{V}_{k}\right)=f_{k}, \quad D_{\mathbf{x}} s\left(\mathbf{V}_{k}\right)=f_{x, k}, \quad D_{\mathbf{y}} s\left(\mathbf{V}_{k}\right)=f_{y, k} .
$$

This interpolation problem turns out to be particularly useful for constructing a local basis for $\mathbb{S}_{2, \mathcal{T}^{*}}^{1}$.

Dierckx [22] presented a geometric method to construct a normalized basis $\left\{B_{i, j, \mathcal{T}^{*}}(\mathbf{X}), j=1,2,3, i=1, \ldots, N_{v}\right\}$ for the spline space $\mathbb{S}_{2, \mathcal{T}^{*}}^{1}$, such that

$$
s(\mathbf{X})=\sum_{i=1}^{N_{v}} \sum_{j=1}^{3} c_{i, j} B_{i, j, \mathcal{T}^{*}}(\mathbf{X})
$$

for all $s \in \mathbb{S}_{2, \mathcal{T}^{*}}^{1}$. Moreover, this basis possesses the following nice properties of univariate B-splines.

- Positivity: $B_{i, j, \mathcal{T}^{*}} \geq 0$.

- Partition of unity: $\sum_{i=1}^{N_{v}} \sum_{j=1}^{3} B_{i, j, \mathcal{T}^{*}}=1$.

- Compact support: $B_{i, j, \mathcal{T}^{*}}$ is zero outside the molecule $\Omega_{i}$ of the vertex $\mathbf{V}_{i}$, which is the subset of $\Omega$ consisting of the points belonging to the union of all triangles of $\mathcal{T}$ containing the vertex $\mathbf{V}_{i}$, see Fig. 30 (right).

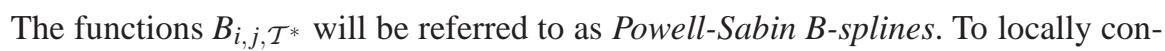

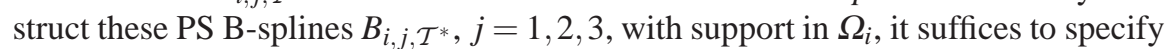
their values and gradients at each vertex of $\mathcal{T}$. Due to the structure of the support $\Omega_{i}$, we have

$$
B_{i, j, \mathcal{T}^{*}}\left(\mathbf{V}_{k}\right)=0, \quad D_{\mathbf{x}} B_{i, j, \mathcal{T}^{*}}\left(\mathbf{V}_{k}\right)=0, \quad D_{\mathbf{y}} B_{i, j, \mathcal{T}^{*}}\left(\mathbf{V}_{k}\right)=0
$$

for any vertex $\mathbf{V}_{k} \neq \mathbf{V}_{i}$. We set

$$
B_{i, j, \mathcal{T}^{*}}\left(\mathbf{V}_{i}\right)=: \alpha_{i, j}, \quad D_{\mathbf{x}} B_{i, j, \mathcal{T}^{*}}\left(\mathbf{V}_{i}\right)=: \beta_{i, j}, \quad D_{\mathbf{y}} B_{i, j, \mathcal{T}^{*}}\left(\mathbf{V}_{i}\right)=: \gamma_{i, j}
$$

We now show how to choose the values $\left(\alpha_{i, 1}, \alpha_{i, 2}, \alpha_{i, 3}\right),\left(\beta_{i, 1}, \beta_{i, 2}, \beta_{i, 3}\right)$ and $\left(\gamma_{i, 1}, \gamma_{i, 2}, \gamma_{i, 3}\right)$ so that the corresponding basis functions, constructed by (101) and (103)-(104), form a convex partition of unity. For each vertex $\mathbf{V}_{i}$, let us consider three points

$$
\left\{\mathbf{Q}_{i, j}:=\left(Q_{i, j, x}, Q_{i, j, y}\right), j=1,2,3\right\}
$$


such that, for $i=1, \ldots, N_{v}$,

$$
\left(\begin{array}{lll}
\alpha_{i, 1} & \alpha_{i, 2} & \alpha_{i, 3} \\
\beta_{i, 1} & \beta_{i, 2} & \beta_{i, 3} \\
\gamma_{i, 1} & \gamma_{i, 2} & \gamma_{i, 3}
\end{array}\right)\left(\begin{array}{lll}
Q_{i, 1, x} & Q_{i, 1, y} & 1 \\
Q_{i, 2, x} & Q_{i, 2, y} & 1 \\
Q_{i, 3, x} & Q_{i, 3, y} & 1
\end{array}\right)=\left(\begin{array}{ccc}
V_{i, x} & V_{i, y} & 1 \\
1 & 0 & 0 \\
0 & 1 & 0
\end{array}\right) .
$$

It follows that the corresponding basis functions $B_{i, j, \mathcal{T}^{*}}$ sum up to one, and that

$$
\mathbf{X}=\sum_{i=1}^{N_{v}} \sum_{j=1}^{3} \mathbf{Q}_{i, j} B_{i, j, \mathcal{T}^{*}}(\mathbf{X})
$$

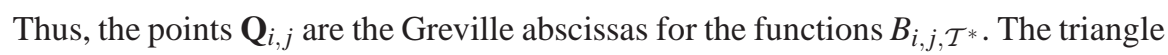
$\mathfrak{T}_{i}:=\left\langle\mathbf{Q}_{i, 1}, \mathbf{Q}_{i, 2}, \mathbf{Q}_{i, 3}\right\rangle$ is referred to as PS triangle associated to the vertex $\mathbf{V}_{i}$.

From (105) it follows that for each vertex $\mathbf{V}_{i}$ the three functions $B_{i, j, \mathcal{T}^{*}, j=}$ $1,2,3$, are uniquely determined by the points $\left\{\mathbf{Q}_{i, j}, j=1,2,3\right\}$. Moreover,

- $\left(\alpha_{i, 1}, \alpha_{i, 2}, \alpha_{i, 3}\right)$ are the barycentric coordinates of the vertex $\mathbf{V}_{i}$ with respect to the triangle $\mathfrak{T}_{i}$;

- $\left(\beta_{i, 1}, \beta_{i, 2}, \beta_{i, 3}\right)$ are the barycentric directional coordinates of the $x$-direction with respect to $\mathfrak{T}_{i}$;

- $\left(\gamma_{i, 1}, \gamma_{i, 2}, \gamma_{i, 3}\right)$ are the barycentric directional coordinates of the $y$-direction with respect to $\mathfrak{T}_{i}$;

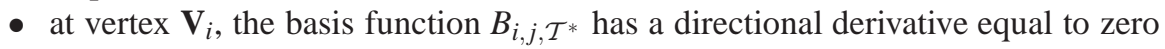
in the direction of the edge of $\mathfrak{T}_{i}$ opposite to $\mathbf{Q}_{i, j}$.

Finally, for each vertex $\mathbf{V}_{i}$ we define its PS points as the vertex itself and the midpoints of all the edges of the PS refinement $\mathcal{T}^{*}$ containing $\mathbf{V}_{i}$, see Fig. 31 . Then we have the following result (see also Fig. 31 and Fig. 32).

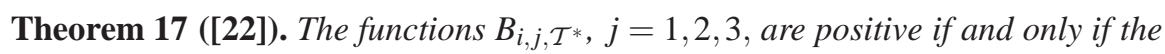
PS triangle $\mathfrak{T}_{i}$ contains all the PS points associated to the vertex $\mathbf{V}_{i}$.

Fig. 31 Location of the PS points (black bullets), and a possible PS triangle for the vertex $\mathbf{V}_{i}$ (shaded), see Theorem 17.

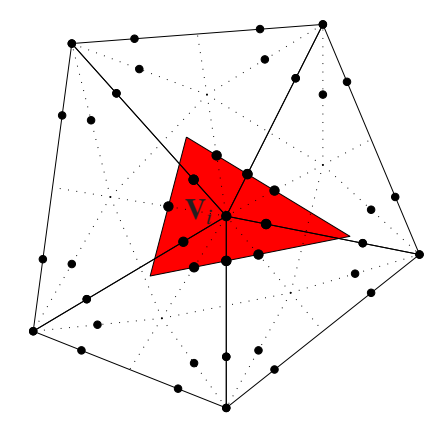

Summarizing, the PS B-splines associated to each vertex $\mathbf{V}_{i}$ of $\mathcal{T}$ are uniquely associated to the triple of points $\mathbf{Q}_{i, j}, j=1,2,3$, forming the PS triangle. Hence, PS triangles can be efficiently used to geometrically identify and describe the PS B-splines and their properties instead of $\alpha_{i, j}, \beta_{i, j}, \gamma_{i, j}$. 

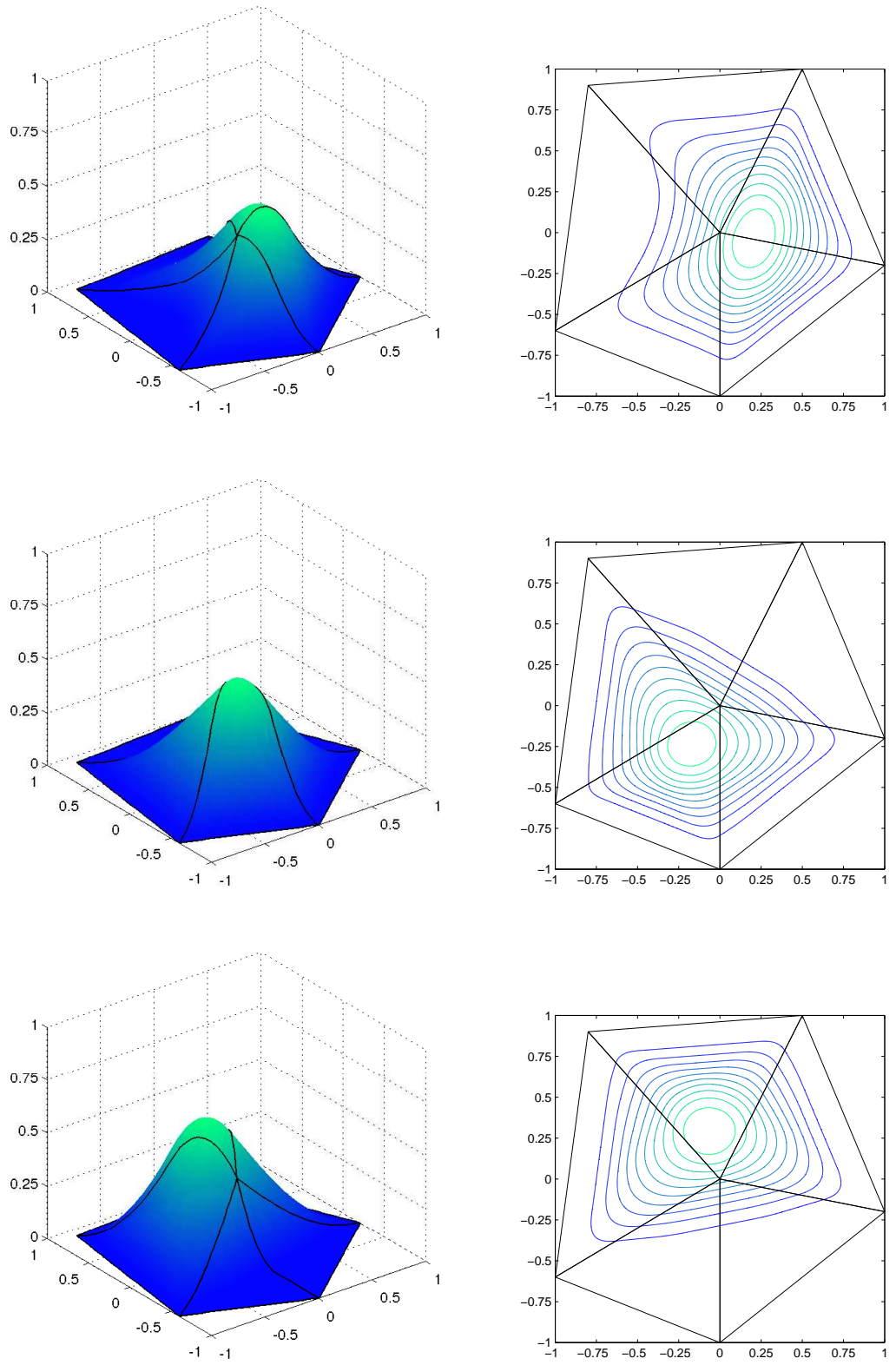

Fig. 32 The three PS B-splines $B_{i, j, \mathcal{T}^{*}}, j=1,2,3$, associated with the vertex $\mathbf{V}_{i}$ and the PS triangle in Fig. 31. Left: the functions $B_{i, j, \mathcal{T}^{*}}$. Right: the contour lines of $B_{i, j, \mathcal{T}^{*}}$. 
Remark 21. Splines of higher degree and smoothness can also be defined on triangulations with a PS refinement, and they can be represented in a similar way as in the quadratic case. We refer to [60] for $C^{2}$ quintics and to [63] for a family of splines with arbitrary smoothness. The quadratic PS case has also been extended to the multivariate setting, see [62].

Remark 22. There also exist similar B-spline representations on triangulations with other macro-structures. For instance, in [61] B-splines have been constructed for the $C^{1}$ cubic reduced Clough-Tocher spline space. In this context, each triangle in the triangulation is subdivided into three subtriangles.

\subsubsection{Geometrical and Graphical Properties}

Combining (102) and (106) leads to the definition of PS control points:

$$
\mathbf{C}_{i, j}=\left(\mathbf{Q}_{i, j}, c_{i, j}\right),
$$

forming the PS control triangles $\left\langle\mathbf{C}_{i, 1}, \mathbf{C}_{i, 2}, \mathbf{C}_{i, 3}\right\rangle$. These triangles are tangent to the spline surface at the vertices $\mathbf{V}_{i}$. The projection of a control triangle onto the $(x, y)$ plane is simply the corresponding PS triangle. Using these control triangles, a designer can interactively change the shape of a given Powell-Sabin spline locally in a predictable way. The positivity and partition of unity properties guarantee that the graph of the spline (102) lies inside the convex hull of its control points $\mathbf{C}_{i, j}$.

For further manipulation (e.g. evaluation and differentiation) of a Powell-Sabin spline in the form (102), we can write the spline in a Bézier-Bernstein representation (Bézier extraction). We consider a reference triangle $T:=\left\langle\mathbf{V}_{1}, \mathbf{V}_{2}, \mathbf{V}_{3}\right\rangle$ with its PS refinement, as shown in Fig. 33. All triangles in $\mathcal{T}$ can be treated in this way. We assume that the points indicated in the figure have the following barycentric coordinates:

$$
\begin{array}{llll}
\mathbf{V}_{1}(1,0,0), & \mathbf{V}_{2}(0,1,0), & \mathbf{V}_{3}(0,0,1), & \mathbf{Z}\left(\zeta_{1}, \zeta_{2}, \zeta_{3}\right), \\
\mathbf{R}_{1}\left(0, \rho_{1}, 1-\rho_{1}\right), & \mathbf{R}_{2}\left(1-\rho_{2}, 0, \rho_{2}\right), & \mathbf{R}_{3}\left(\rho_{3}, 1-\rho_{3}, 0\right)
\end{array}
$$

On each of the six subtriangles in $T$ the Powell-Sabin spline is a quadratic polynomial, that can be represented in its Bézier-Bernstein formulation, i.e., with $p=2$ in equations (77) and (78). The value of the corresponding Bézier ordinates is derived in [22]. The outcome is schematically represented in Fig. 34, with

$$
\begin{aligned}
& v_{1}=\alpha_{1,1} c_{1,1}+\alpha_{1,2} c_{1,2}+\alpha_{1,3} c_{1,3}, \\
& s_{1}=\sigma_{1,1} c_{1,1}+\sigma_{1,2} c_{1,2}+\sigma_{1,3} c_{1,3}, \\
& s_{1}^{\prime}=\sigma_{1,1}^{\prime} c_{1,1}+\sigma_{1,2}^{\prime} c_{1,2}+\sigma_{1,3}^{\prime} c_{1,3}, \\
& \tilde{s}_{1}=\widetilde{\sigma}_{1,1} c_{1,1}+\tilde{\sigma}_{1,2} c_{1,2}+\widetilde{\sigma}_{1,3} c_{1,3} .
\end{aligned}
$$

Like $\left(\alpha_{1,1}, \alpha_{1,2}, \alpha_{1,3}\right)$ are the barycentric coordinates of the vertex $\mathbf{V}_{1}$, the triplets $\left(\sigma_{1,1}, \sigma_{1,2}, \sigma_{1,3}\right),\left(\sigma_{1,1}^{\prime}, \sigma_{1,2}^{\prime}, \sigma_{1,3}^{\prime}\right)$ and $\left(\widetilde{\sigma}_{1,1}, \widetilde{\sigma}_{1,2}, \widetilde{\sigma}_{1,3}\right)$ are found as the barycentric 


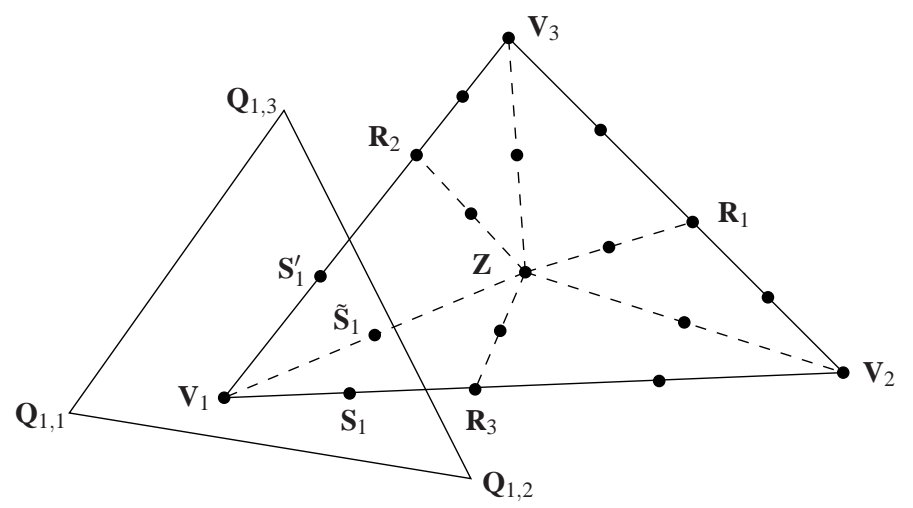

Fig. 33 PS refinement of a reference triangle $T:=\left\langle\mathbf{V}_{1}, \mathbf{V}_{2}, \mathbf{V}_{3}\right\rangle$, together with the PS triangle $\mathfrak{T}_{1}:=\left\langle\mathbf{Q}_{1,1}, \mathbf{Q}_{1,2}, \mathbf{Q}_{1,3}\right\rangle$ associated with the vertex $\mathbf{V}_{1}$.

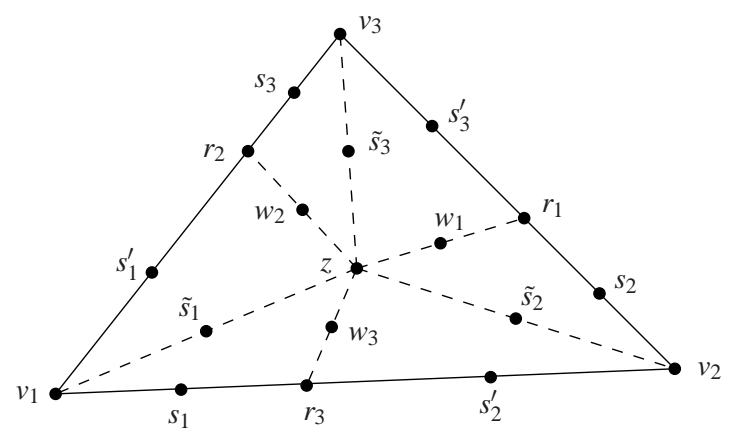

Fig. 34 Schematic representation of the Bézier ordinates of a Powell-Sabin spline.

coordinates of the PS points $\mathbf{S}_{1}, \mathbf{S}_{1}^{\prime}$ and $\tilde{\mathbf{S}}_{1}$, respectively, with respect to the PS triangle $\mathfrak{T}_{1}$. These points are depicted in Fig. 33. Analogously, we can compute the values of $\left(v_{2}, s_{2}, s_{2}^{\prime}, \tilde{s}_{2}\right)$ and $\left(v_{3}, s_{3}, s_{3}^{\prime}, \tilde{s}_{3}\right)$. The other Bézier ordinates are derived from the inherent continuity conditions of the Powell-Sabin spline, e.g.,

$$
\begin{aligned}
r_{3} & =\rho_{3} s_{1}+\left(1-\rho_{3}\right) s_{2}^{\prime}, \\
w_{3} & =\rho_{3} \tilde{s}_{1}+\left(1-\rho_{3}\right) \tilde{s}_{2}, \\
z & =\zeta_{1} \tilde{s}_{1}+\zeta_{2} \tilde{s}_{2}+\zeta_{3} \tilde{s}_{3} .
\end{aligned}
$$

In this Bézier-Bernstein representation the Powell-Sabin spline can be easily manipulated using the de Casteljau algorithm, see (93) with $p=2$. 
Being equipped with a B-spline-like basis, PS splines admit a straightforward rational extension.

Definition 19. A NURPS (Non-Uniform Rational PS) surface on a PS refinement $\mathcal{T}^{*}$ of a triangulation $\mathcal{T}$ of $\Omega$ is given by

$$
\mathcal{S}(\mathbf{X})=\sum_{i=1}^{N_{v}} \sum_{j=1}^{3} \mathbf{C}_{i, j} R_{i, j, \mathcal{T}^{*}}(\mathbf{X}), \quad R_{i, j, \mathcal{T}^{*}}(\mathbf{X}):=\frac{w_{i, j} B_{i, j, \mathcal{T}^{*}}(\mathbf{X})}{\sum_{l=1}^{N_{v}} \sum_{r=1}^{3} w_{l, r} B_{l, r, \mathcal{T}^{*}}(\mathbf{X})},
$$

where $\mathbf{C}_{i, j} \in \mathbb{R}^{d}$ are called NURPS control points, $B_{i, j, \mathcal{T}^{*}}$ are the normalized PS $B$-splines, and $w_{i, j}$ are strictly positive weights.

With particular choices for the control points and weights, NURPS can exactly represent patches on quadric surfaces. The influence of the weights on a NURPS surface can be described in a geometrically intuitive way [65].

As for classical NURBS, see (63), it is sometimes useful to consider the so-called homogeneous representation of (107), in which the NURPS surface is decoupled into $d+1$ standard PS spline components, i.e.

$$
\left(\sum_{i=1}^{N_{v}} \sum_{j=1}^{3} \mathbf{C}_{i, j} w_{i, j} B_{i, j, \mathcal{T}^{*}}(\mathbf{X}), \sum_{i=1}^{N_{v}} \sum_{j=1}^{3} w_{i, j} B_{i, j, \mathcal{T}^{*}}(\mathbf{X})\right)
$$

Remark 23. NURPS surfaces can be represented in a Bézier-Bernstein formulation by means of rational Bézier ordinates [69]. They can then be evaluated and manipulated in a stable way by using the rational de Casteljau algorithm [25, Chapter 18].

\section{Conclusions}

We have provided an extended summary of the most popular tools in CAGD, namely Bernstein polynomials and B-splines, from the IgA perspective. We have paid special attention to the reason why such representations are so popular and efficient. In particular, we have proved that they are the best bases to represent polynomials or piecewise polynomials, respectively.

We also have reviewed some generalizations of the B-spline representation to a large class of spaces which can be of interest in IgA, namely the so-called generalized B-splines. Generalized B-splines can be used to exactly represent geometries of interest in applications, like conic sections, avoiding the use of rational forms. In addition, they can be exploited according to a problem-oriented discretization perspective, see [18, 43, 44, 45].

Finally, we have summarized the extension of the B-spline structure to the Powell-Sabin spline space, i.e. a suitable spline space defined on triangulations with a particular macro-structure. The triangular setting naturally supports local mesh refinements. Therefore, Powell-Sabin splines can be an interesting tool in $\operatorname{IgA}$, see $[64,66,67]$. 


\section{References}

1. Ando, T.: Totally positive matrices. Linear Algebra Appl. 90, 165-219 (1987)

2. Bernstein, S. N.: Démonstration du théorème de Weierstrass fondée sur le calcul des probabilités. Communications de la Société Mathématique de Kharkov 2. Series XIII, 1-2 (1912)

3. Bézier, P.: Mathematical and practical possibilities of UNISURF. In: Barnhill, R. E., Riesenfeld, R. F. (eds.), Computer Aided Geometric Design, pp. 127-152. Academic Press, New York (1974)

4. Böhm, W., Farin, G., Kahmann, J.: A survey of curve and surface methods in CAGD. Comput. Aided Geom. Design 1, 1-60 (1984)

5. Böhm, W., Müller, A.: On de Casteljau's algorithm. Comput. Aided Geom. Design 16, 587605 (1999)

6. de Boor, C.: On calculating with B-splines. J. Approx. Theory 6, 50-62 (1972)

7. de Boor, C., Höllig, K., Riemenschneider, S.: Box splines. Springer-Verlag (1993)

8. de Boor, C.: A Practical Guide to Splines. Springer, Revised edition (2001)

9. de Boor, C.: The way things were in multivariate splines: A personal view. In: DeVore, R., Kunoth, A. (eds.), Multiscale, Nonlinear and Adaptive Approximation, pp. 19-37. SpringerVerlag (2009)

10. Bosner, T., Rogina, M.: Non-uniform exponential tension splines. Numer. Algor. 46, 265-294 (2007)

11. Butzer, P. L., Schmidt, M., Stark, E. L.: Observations on the history of central B-splines. Arch. History Exact Sci. 39, 137-156 (1988)

12. Carnicer, J. M., Peña, J. M.: Shape preserving representations and optimality of the Bernstein basis. Adv. Comp. Math. 1, 173-196 (1993)

13. Carnicer, J. M., Peña, J. M.: Totally positive bases for shape preserving curve design and optimality of B-splines. Comput. Aided Geom. Design 6, 633-654 (1994)

14. Carnicer, J. M., Peña, J. M.: Totally positivity and optimal bases. In: Gasca, M., Micchelli, C. A. (eds.), Total Positivity and its Applications, pp. 133-155. Kluwer, Dordrecht (1996)

15. de Casteljau, P.: De Casteljau's autobiography: My time at Citroën. Comput. Aided Geom. Design 16, 583-586 (1999)

16. Cohen, E., Lyche, T., Riesenfeld, R.: Discrete B-splines and subdivision techniques in computer-aided geometric design and computer graphics. Comput. Graph. Image Process. 14, 87-111 (1980)

17. Costantini, P., Lyche, T., Manni, C.: On a class of weak Tchebycheff systems. Numer. Math. 101, 333-354 (2005)

18. Costantini, P., Manni, C., Pelosi, F., Sampoli, M. L.: Quasi-interpolation in isogeometric analysis based on generalized B-splines. Comput. Aided Geom. Design 27, 656-668 (2010)

19. Cottrell, J. A., Hughes, T. J. R., Bazilevs, Y.: Isogeometric Analysis: Toward Integration of CAD and FEA. John Wiley \& Sons (2009)

20. Curry, H. B., Schoenberg, I. J.: On spline distributions and their limits: The Pólya distribution functions. Bull. Amer. Math. Soc. 53, 1114, Abstract 380t (1947)

21. Dahmen, W., Micchelli, C. A., Seidel, H.-P.: Blossoming begets B-splines built better by B-patches. Math. Comp. 59, 97-115 (1992)

22. Dierckx, P.: On calculating normalized Powell-Sabin B-splines. Comput. Aided Geom. Design 15, 61-78 (1997)

23. Dyn, N., Levin, D.: Subdivision schemes in geometric modelling. Acta Numer. 11, 73-144 (2002)

24. Dyn, N., Levin, D., Luzzatto, A.: Exponentials reproducing subdivision schemes. Found. Comput. Math. 3, 187-206 (2003)

25. Farin, G.: Curves and surfaces for computer aided geometric design. A practical guide. Fifth edition. Academic Press, Boston (2002)

26. Farouki, R. T.: The Bernstein polynomial basis: a centennial retrospective. Comput. Aided Geom. Design 29, 379-419 (2012) 
27. Farouki, R. T., Goodman, T. N. T.: On the optimal stability of the Bernstein basis. Math. Comp. 65, 1553-1566 (1996)

28. Fekete, M., Pólya, G.: Über ein Problem von Laguerre. Rend. Circ. Matem. Palermo 34, 89120 (1912)

29. Gasca, M., Micchelli, C. A. (eds.): Total Positivity and its Applications. Kluwer, Dordrecht (1996)

30. Gasca, M., Peña, J. M.: On the characterization of totally positive matrices In: Singh, S. P. (ed.), Approximation Theory, spline functions and applications, pp. 357-364. Kluwer Academic Publishers, Dordrecht (1992)

31. Goodman, T. N. T.: Total positivity and shape of curves. In: Gasca, M., Micchelli, C. A. (eds.), Total Positivity and its Applications, pp. 157-186. Kluwer, Dordrecht (1996)

32. Goodman, T. N. T., Mazure, M. L.: Blossoming beyond extended Chebyshev spaces. J. Approx. Theory 109, 48-81 (2001)

33. Greville, T. N. E.: On the normalisation of the B-splines and the location of the nodes for the case of unequally spaced knots. In: Shisha, O. (ed.), Inequalities, pp. 286-290. Academic Press, New York (1967)

34. Hoschek, J., Lasser, D.: Fundamentals of Computer Aided Geometric Design. A K Peters (1993)

35. Karlin, S.: Total Positivity. Stanford University Press, Stanford (1968)

36. Karlin, S.: To I. J. Schoenberg and his mathematics. J. Approx. Theory 8, vi-ix (1973)

37. Koch, P. E., Lyche, T.: Interpolation with exponential B-splines in tension. In: Farin, G., Hagen, H., Noltemeier, H., Knödel, W. (eds.), Geometric modelling, pp. 173-190. SpringerVerlag, London (1993)

38. Kvasov, B., Sattayatham, P.: GB-splines of arbitrary order. J. Comput. Appl. Math. 104, 63-88 (1999)

39. Lai, M.-J., Schumaker, L. L.: Spline Functions on Triangulations. Cambridge U. P. (2007)

40. Laurent, P.-J., Sablonnière, P.: Pierre Bézier: An engineer and a mathematician. Comput. Aided Geom. Design 18, 609-617 (2001)

41. Lyche, T.: A recurrence relation for Chebyshevian B-splines. Constr. Approx. 1, 155-173 (1985)

42. Mainar, E., Peña, J. M.: A general class of Bernstein-like bases. Comput. Math. Appl. 53, 1686-1703 (2007)

43. Manni, C., Pelosi, F., Sampoli, M. L.: Generalized B-splines as a tool in isogeometric analysis. Comput. Methods Appl. Mech. Engrg. 200, 867-881 (2011)

44. Manni, C., Pelosi, F., Sampoli, M. L.: Isogeometric analysis in advection-diffusion problems: tension splines approximation. J. Comput. Appl. Math. 236, 511-528 (2011)

45. Manni, C., Pelosi, F., Speleers, H.: Local hierarchical $h$-refinements in IgA based on generalized B-splines. In: Floater, M. et al. (eds.), Mathematical Methods for Curves and Surfaces 2012, LNCS 8177, pp. 341-363. Springer-Verlag (2014)

46. Mazure, M. L.: Chebyshev-Bernstein bases. Comput. Aided Geom. Design 16, 649-669 (1999)

47. Mazure, M. L.: Chebyshev splines beyond total positivity. Adv. Comp. Math. 14, 129-156 (2001)

48. Mazure, M. L.: How to build all Chebyshevian spline spaces good for geometric design? Numer. Math. 119, 517-556 (2011)

49. Neamtu, N.: What is the natural generalization of univariate splines to higher dimensions? In: Lyche, T., Schumaker, L. L. (eds.), Mathematical Methods for Curves and Surfaces, pp. 355-392. Vanderbilt University Press, Nashville (2001)

50. Neamtu, N.: Delaunay configurations and multivariate splines: A generalization of a result of B. N. Delaunay. Trans. Amer. Math. Soc. 359, 2993-3004 (2007)

51. Peña, J. M. (ed.): Shape Preserving Representations in Computer-Aided Geometric Design. Nova Science Publishers, New York (1999)

52. Piegl, L., Tiller, W.: The NURBS Book (Monographs in Visual Communication), 2nd edition. Springer-Verlag, New York (1997) 
53. Powell, M. J. D., Sabin, M. A.: Piecewise quadratic approximations on triangles. ACM Trans. Math. Software 3, 316-325 (1977)

54. Prautzsch, H., Boehem, W., Paluszny, M.: Bézier and B-spline Techniques. Springer (2002)

55. Prautzsch, H., Gallagher, T.: Is there a geometric variation diminishing property for B-spline or Bézier surfaces? Comput. Aided Geom. Design 9, 119-124 (1992)

56. Ramshaw, L.: Blossoms are polar forms. Comput. Aided Geom. Design 6, 323-358 (1989)

57. Schoenberg, I. J.: On spline functions. In: Shisha, O. (ed.), Inequalities, pp. 255-286. Academic Press, New York (1967)

58. Schoenberg, I. J.: A brief account of my life and work. In: de Boor, C. (ed.), I. J. Schoenberg, Selected Papers, Volume I, pp. 1-10. Birkhäuser, Boston (1988)

59. Schumaker, L. L.: Spline Functions: Basic Theory, third edition. Cambridge U. P. (2007)

60. Speleers, H.: A normalized basis for quintic Powell-Sabin splines. Comput. Aided Geom. Design 27, 438-457 (2010)

61. Speleers, H.: A normalized basis for reduced Clough-Tocher splines. Comput. Aided Geom. Design 27, 700-712 (2010)

62. Speleers, H.: Multivariate normalized Powell-Sabin B-splines and quasi-interpolants. Comput. Aided Geom. Design 30, 2-19 (2013)

63. Speleers, H.: Construction of normalized B-splines for a family of smooth spline spaces over Powell-Sabin triangulations. Constr. Approx. 37, 41-72 (2013)

64. Speleers, H., Dierckx, P., Vandewalle S.: Numerical solution of partial differential equations with Powell-Sabin splines. J. Comput. Appl. Math. 189, 643-659 (2006)

65. Speleers, H., Dierckx, P., Vandewalle S.: Weight control for modelling with NURPS surfaces. Comput. Aided Geom. Design 24, 179-186 (2007)

66. Speleers, H., Manni, C., Pelosi, F.: From NURBS to NURPS geometries. Comput. Methods Appl. Mech. Engrg. 255, 238-254 (2013)

67. Speleers, H., Manni, C., Pelosi, F., Sampoli, M. L.: Isogeometric analysis with PowellSabin splines for advection-diffusion-reaction problems. Comput. Methods Appl. Mech. Engrg. 221-222, 132-148 (2012)

68. Wang, G., Fang, M.: Unified and extended form of three types of splines. J. Comput. Appl. Math. 216, 498-508 (2008)

69. Windmolders, J., Dierckx, P.: From PS-splines to NURPS. In: Cohen, A., Rabut, C., Schumaker, L. L. (eds.), Proceedings of Curve and Surface Fitting, Saint-Malo, 1999, pp. 45-54. Vanderbilt University Press (2000)

70. Zhang, J., Krause, F., Zhang, H.: Unifying C-curves and H-curves by extending the calculation to complex numbers. Comput. Aided Geom. Design 22, 865-883 (2005) 